University of Louisville

ThinkIR: The University of Louisville's Institutional Repository

$12-2013$

\title{
Testing location memory for threatening and nonthreatening stimuli : implications for evolutionary psychological theorizing.
}

Ryan Patrick Hacklander

University of Louisville

Follow this and additional works at: https://ir.library.louisville.edu/etd

\section{Recommended Citation}

Hacklander, Ryan Patrick, "Testing location memory for threatening and nonthreatening stimuli : implications for evolutionary psychological theorizing." (2013). Electronic Theses and Dissertations. Paper 558.

https://doi.org/10.18297/etd/558

This Doctoral Dissertation is brought to you for free and open access by ThinkIR: The University of Louisville's Institutional Repository. It has been accepted for inclusion in Electronic Theses and Dissertations by an authorized administrator of ThinkIR: The University of Louisville's Institutional Repository. This title appears here courtesy of the author, who has retained all other copyrights. For more information, please contact thinkir@louisville.edu. 
TESTING LOCATION MEMORY FOR THREATENING AND NONTHREATENING STIMULI: IMPLICATIONS FOR EVOLUTIONARY PSYCHOLOGICAL THEORIZING

\author{
By \\ Ryan Patrick Hackländer \\ B.S., Kentucky Wesleyan College, 2008 \\ M.S., University of Louisville, 2010

\begin{abstract}
A Dissertation
Submitted to the Faculty of the

College of Arts and Sciences of the University of Louisville in Partial Fulfillment of the Requirements

for the Degree of
\end{abstract} \\ Doctor of Philosophy \\ Department of Psychological and Brain Sciences \\ University of Louisville \\ Louisville, Kentucky
}

December, 2013 

TESTING LOCATION MEMORY FOR THREATENING AND NONTHREATENING STIMULI: IMPLICATIONS FOR EVOLUTIONARY PSYCHOLOGICAL THEORIZING

\author{
By \\ Ryan Patrick Hackländer \\ B.S., Kentucky Wesleyan College, 2008 \\ M.S., University of Louisville, 2010 \\ A Dissertation Approved on \\ October 11, 2013 \\ by the following Dissertation Committee:
}

\begin{tabular}{c}
\hline Keith B. Lyle \\
Dissertation Director \\
\hline Cara Cashon \\
\hline Fabian Crespo \\
\hline Marci DeCaro \\
\hline
\end{tabular}

Tamara Newton 


\section{DEDICATION}

I dedicate this dissertation to Aoidê, Meletê, and Mnêmê.

I dedicate this dissertation to Rochina Cipriani.

To me they are the same.

To think on her: "Questo é amore" 


\section{ACKNOWLEDGMENTS}

Although I know that people typically don't take the time to read the acknowledgments, and many feel that they are unimportant, I believe there is a good reason they are included, which has nothing to do with convention. It lies in the fact that no one can achieve what they do without the assistance of others. Those people deserve to be acknowledged. Therefore, I will not be limiting my acknowledgment section for reasons of space preservation.

I would like to begin with the Beguine. In doing so, the first person deserving of my acknowledgment is my loving wife. She has been religious in her devotion to the completion of my degree, sacrificing much. She has also been on this slow dance for the complete duration, and I believe she is nearly as excited as I am that the music has finally faded and we can step off the dance floor. I am excited to begin a post-dissertation life with her.

I would also like to thank my extended family, including my in-laws. Each one of them has continued to show an interest in my progress. In addition to this interest, their positivity and support has helped keep me motivated. I am grateful for their attention and kindness.

I would like to acknowledge my mother in law, Martina Hackländer. Ihre Hilfsbereitschaft hat mir sehr viel bedeutet und es mir vereinfacht nach Deutschland zu ziehen. Außerdem haben meine vielen Gespräche mit ihr es mir nicht nur vereinfacht die deutsche Sprache besser zu lernen, sondern mir auch immer wieder positiven Ansporn gegeben. The sun shines bright and we can say, it is a lovely day today! Danke Mama. 
I would certainly like to thank the members of my dissertation committee, Cara Cashon, Fabian Crespo, Marci DeCaro, and Tamara Newton for all the effort and time they have put into helping me get this far. Aside from being part of my committee they have also been teachers and mentors to me during my time at the University of Louisville. They are all people I respect and admire. Also, thanks to Fabian I will never again underestimate the power of randomness in either evolution or life in general.

Another person at the University of Louisville who has had a large impact on my progression has been my lab mate and friend James Matthew Edlin. I am not sure I can say I would have been able to make it through the program without him. If ever I had a question about anything he would be the person I would ask. He has also expanded my conception of science with our many conversations, especially those about thin spaces.

This almost goes without saying, but the person at the University of Louisville who has had the greatest influence on me has been my mentor, Keith Lyle. My wife and I have both on occasion remarked that I "lucked out" with getting Keith as my mentor. Although Fortuna was never one of the goddesses I held in high esteem, this case may be the one exception. Keith has been everything a mentor should be: pushy and patient, critical and kind, knowledgeable, and enthusiastic. The impact he has had on my progression as a researcher and writer is too immense to put into words. I can think of no other way to acknowledge his help and impact than to say this dissertation is only possible because of him.

To several of the current and former members of Kentucky Wesleyan College I am also grateful. To Dr. David Oetinger for stressing the importance of precision in all 
things; to Doctors Tony Lee and Kurt Knoll for showing me the paradox of a boundless mind existing within a finite and bound universe; to Dr. Justin Rosemeier for sharing my interests in natural selection and a certain refined way of languaging with one another; to Dr. Lynette Taylor for introducing me to so many wondrous topics; to Inge Schleiden weil sie immer für mich da war; and last, but certainly not least, to Dr. Bernie Bettinelli for doing nothing less than kindling my interest in learning.

My grandmother, Carol Balvin, has also had a huge impact on me. She has always been extremely kind, and is living proof that love can be unconditional. I cannot adequately express how glad I am of all the support she has given me over the years. I hope she knows how important all her care has been to any accomplishments I may have made throughout my life, including the completion of this dissertation.

My sister, and the namesake of my muse, Rochina Mulvaney has unknowingly constantly pushed me to succeed. When we were growing up this sweet little girl always looked up to me as a role model and someone who she wanted to be. She does not know it, but anything good or useful I have ever done has been solely the result of the fact that I could never allow myself to disappoint that girl.

My mother has had the greatest influence on my education throughout my entire life. From reading to me every night when I was little, to coming on school field trips, to discussing politics and whatever interesting topic I learned about during the day in college, she has always been there to support me and keep my mind active. Also, she has somehow made me feel as though there was no other option except to keep going straight from high school to college and beyond; this is something that I have only recently come 
to acknowledge as seeming strange to other people, but I have never known any different and I am very happy about that. I hope that I have made her proud.

Finally, I would like to acknowledge my father. He, as a skeptic in the antiquated sense of the word, has at every moment of my life challenged me to, as Paul Simon said, "know what I know". He has also kept me focused by reminding me that I can "gather all the news I need from the weather report" and that "you don't need a weather man to know which way the wind blows". Although I am not sure that I can ever know what I know, I think I have a pretty good grasp of what comes in the following pages. I hope that will suffice for the time being. 


\begin{abstract}
TESTING LOCATION MEMORY FOR THREATENING AND NONTHREATENING

STIMULI: IMPLICATIONS FOR EVOLUTIONARY PSYCHOLOGICAL
\end{abstract}

THEORIZING

Ryan P. Hackländer

October 11, 2013

Humans respond to the presence of threatening stimuli more rapidly than nonthreatening stimuli, a trait that some authors believe humans have been selected for. Based on this finding, it has been proposed that humans should also have superior location memory for threatening stimuli, possibly depending on whether stimuli have ancestral (e.g., snakes) or modern (e.g., guns) ecological relevance. This is herein called the Superior Location Memory for Threatening Stimuli (SLMTS) hypothesis. Some authors believe that humans possess a domain-specific adaptation that gives rise to the hypothesized memory advantage for threatening stimuli. The primary aim of this dissertation is to test the SLMTS hypothesis. Three experiments were performed using stimuli that fully crossed threat level (threatening versus nonthreatening) and ecological relevance (ancestral versus modern). Each experiment included a learning phase, in which subjects responded to threatening or nonthreatening stimuli in various locations, and a subsequent location memory phase. Experiments 1 and 2 tested explicit location memory. Experiment 1 compared recall and recognition tests of conscious location memory. Experiment 2 used a version of the Process Dissociation Procedure to test both conscious and unconscious influences of location memory. Location memory for ancestral nonthreatening, ancestral threatening, and modern threatening stimuli was better 
than for modern nonthreatening stimuli. These results do not support the SLMTS hypothesis but rather support the general mnemonic principles (GMP) hypothesis, which is that location memory is best for stimuli that are uncommon, arousing, and valenced (either positive or negative). However, Experiment 3 tested implicit location memory and supported the SLMTS hypothesis: Implicit memory was greater for threatening than nonthreatening stimuli.

I argue that, taken together, the results of the three experiments do not require the invocation of a specific adaptation for explanatory purposes. Finding support for the GMP hypothesis in Experiments 1 and 2 and the SLMTS hypothesis in Experiment 3 is consistent with a domain-general explanation: Location memory is best for stimuli that are deemed most relevant to the memory system given current circumstances and goals. The relevance of these findings to evolutionary psychological theories of memory is discussed and suggestions for future research are offered. 


\section{TABLE OF CONTENTS}

PAGE

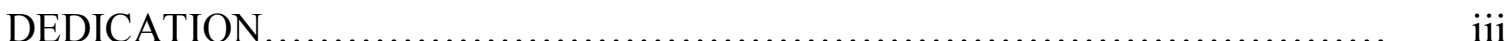

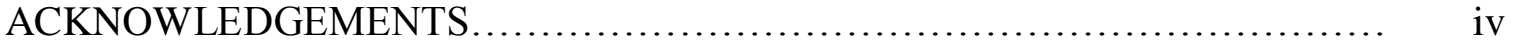

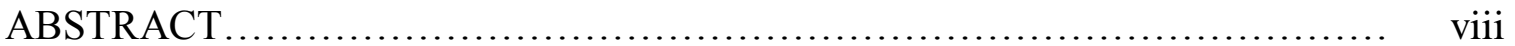

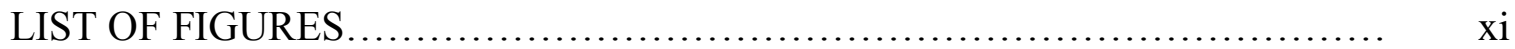

INTRODUCTION ........................................................ 1

Hypotheses................................................... 25

Introduction to Experiments....................................... 34

GENERAL METHODS .................................................... 36

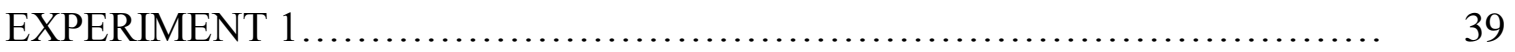

Method....................................................... 41

Results.......................................................... 43

Discussion......................................................... 54

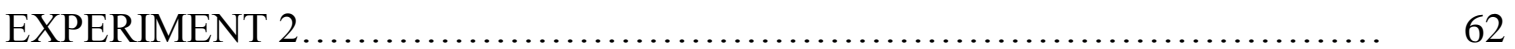

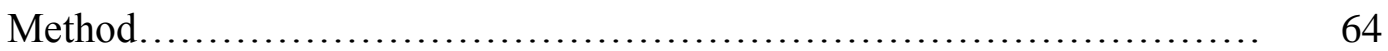

Results....................................................... 67

Discussion.......................................................... 77

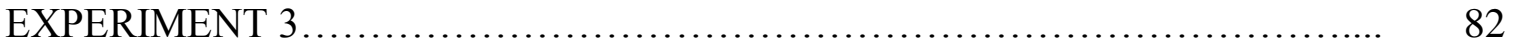

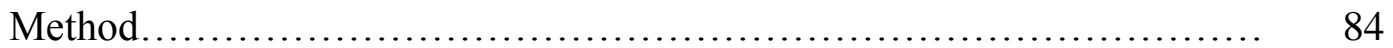

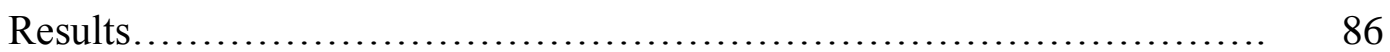

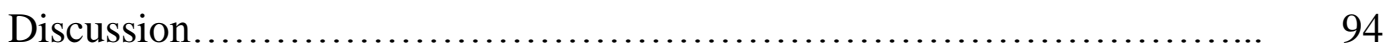

GENERAL DISCUSSION ................................................ 96

Hypotheses...................................................... 96

Natural Selection as Unifying Factor................................. 105

Differences between Attention and Memory........................... 107

Relation to Past Research.......................................... 107

Future Directions................................................. 110

Conclusions...................................................... 113

ENDNOTES............................................................ 115

REFERENCES ........................................................ 124

APPENDICES ....................................................... 140

CURRICULUM VITAE .................................................. 144 


\section{LIST OF FIGURES}

$\begin{array}{lll}\text { FIGURE } & \text { PAGE }\end{array}$

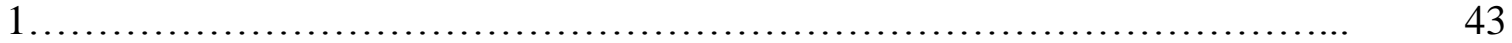

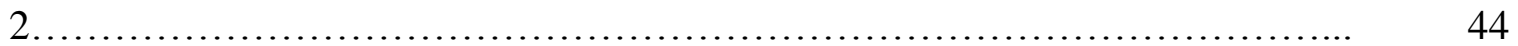

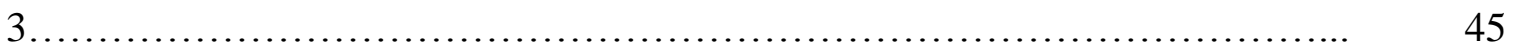

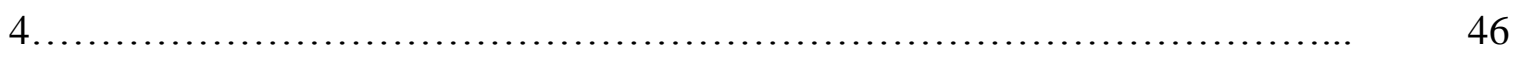

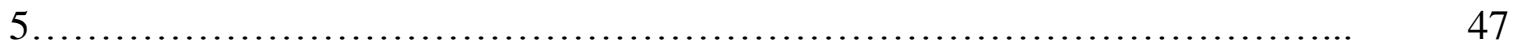

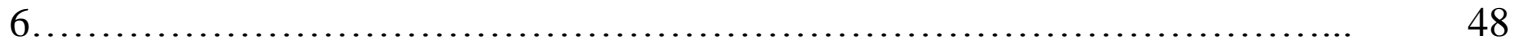

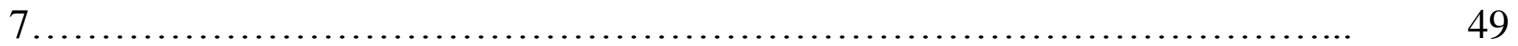

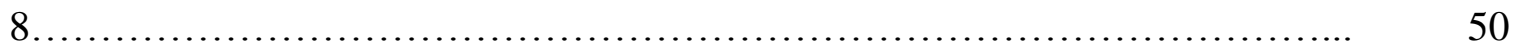

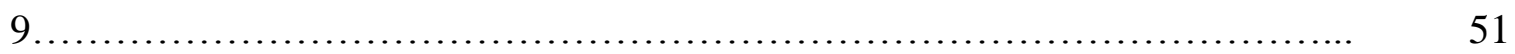

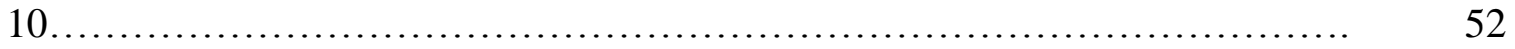

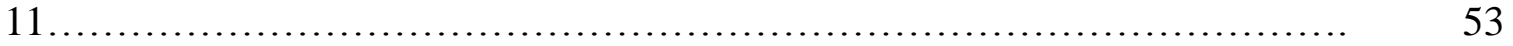

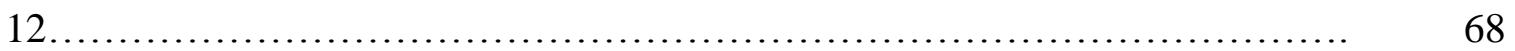

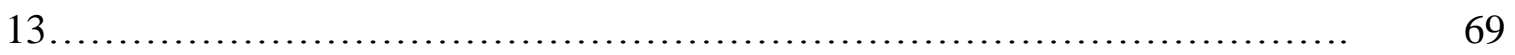

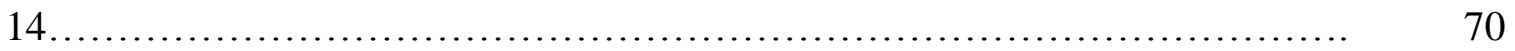

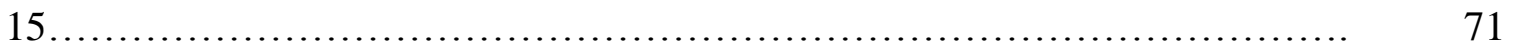

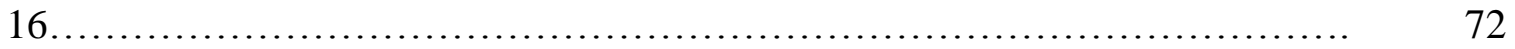

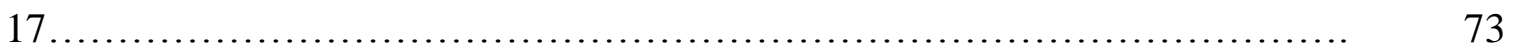

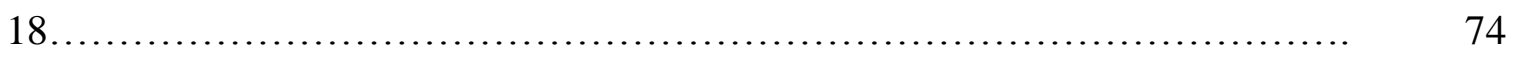

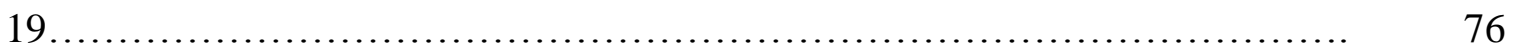

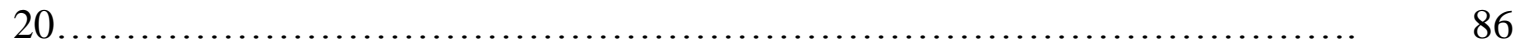

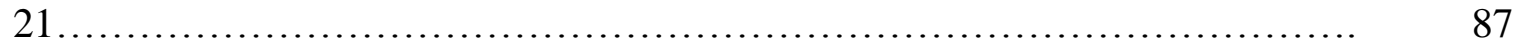

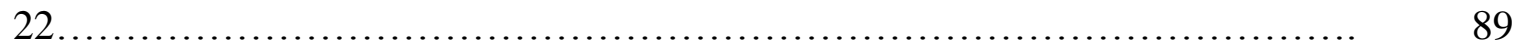

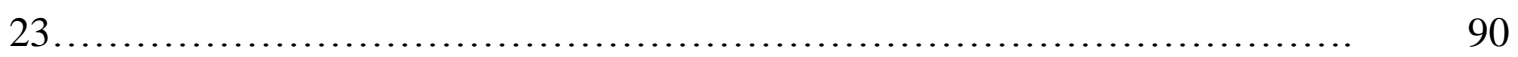

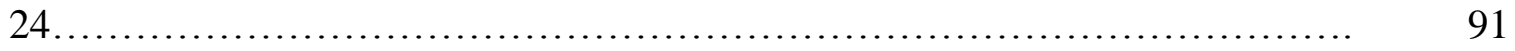

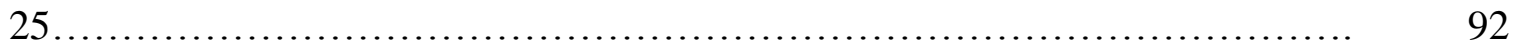




\section{INTRODUCTION}

Since life first appeared on the planet each species has had to deal with recurrent problems posed by environmental factors and other animals (predator, prey, or competitor) that have shaped and continue to shape their physical and mental characteristics. Humans are no exception. One such problem that humans have faced throughout the course of evolution is the challenge of detecting and avoiding predators and other threatening stimuli and situations, such as venomous snakes and spiders or large, territorially defensive animals. Recognition of this recurrent problem, coupled with knowledge of the theory of natural selection ${ }^{1}$, has led some authors to argue that humans' location memory system is specifically tuned to remember those stimuli and locations which are most important to our survival (e.g., Nairne, VanArsdall, Pandeirada, \& Blunt, 2012).

Psychology has been influenced by the theory of natural selection ever since Darwin first published On the Origin of Species in 1859. For example, Carl Jung's ideas of collective unconscious and instincts relied heavily on Darwinian thinking (Hogenson, 2001). Also, Darwin's ideas about evolution influenced the way William James conceptualized human knowledge and morality (Skrupskelis, 2007). Although there is a long historical connection between psychology and the theory of evolution, recent decades have seen an increase in attempts to explain psychological phenomena from an evolutionary perspective (Cosmides \& Tooby, 2013). Studying phenomena from an evolutionary perspective involves applying what is known about the selection pressure 
faced by human ancestors to generate hypotheses about human behavior. These hypotheses are then tested using the experimental method. This field is typically known as evolutionary psychology (Cosmides, Tooby, \& Barkow, 1992), but this name may not be the most accurate or descriptive. Research in this area has been critiqued for making exclusively adaptationist arguments, or reducing evolution down to only the force of natural selection (Gould \& Lewontin, 1979) ${ }^{2}$. Nonetheless, this dissertation will continue to use the term evolutionary psychology for the sake of continuity with past literature.

In relation to human location memory, an evolutionary psychological argument looks broadly as follows. Throughout the course of evolution, humans have lived in an environment containing many different stimulus types, with varying degrees of survival relevance. Stimuli which were threatening to humans' survival were particularly important. Ancestral humans varied in their location memory, including some individuals who remembered the locations of threatening stimuli better than the locations of nonthreatening stimuli. This variability in location memory was, to some degree, heritable. Individuals with superior memory for the locations of threatening stimuli could avoid these stimuli in the future and were less likely to encounter them a second time. Therefore, they tended to survive for longer periods. Because they were more likely to survive to older ages, they were also more likely to reach reproductive maturity, find a mate, and actually reproduce, all compared to other humans with inferior memory for threatening stimuli. Humans with superior location memory for threatening stimuli passed this trait ${ }^{3}$ down to future generations. This continued throughout human history up to recent times. Therefore, modern humans should also have enhanced location 
memory for threatening stimuli, as compared to nonthreatening stimuli, due to the selection pressures faced by our ancestors.

The idea that location memory should be better for threatening than nonthreatening stimuli is herein called the Superior Location Memory for Threatening Stimuli (SLMTS) hypothesis. Aside from the adaptationist reasoning described in the previous paragraph, there are other reasons to believe that location memory might be better for threatening than nonthreatening stimuli. These reasons stem from findings on detection of and attention to threatening stimuli, prepared learning, memory for emotional stimuli, memory in survival contexts, and memory for cheaters. A brief overview of these topics, and how they relate to location memory for threatening stimuli, is presented below.

\section{Detection and Attention Research}

Research on human attention to threatening stimuli has flourished in the field of evolutionary psychology. Two lines of research have been most prominent. One is detection of and attention to faces that signal threat or the presence of threat. The second is detection of and attention to other threatening stimuli, such as predators. Both are discussed below.

Before reviewing past research, a brief discussion of terminology is necessary because the terminology used in this dissertation will differ from what is used in much other research. Specifically, during the learning phase of the current experiments (described below), the main dependent variable will be called response time, instead of

detection time. Previous research has commonly claimed that humans detect threatening 
stimuli more rapidly than nonthreatening stimuli (Fox, Griggs, \& Mouchlianitis, 2007). While this may be the case, the argument can be made that this claim has not actually been tested because all previous research has relied on behavioral responses to measure detection times and these do not isolate the time required to detect a stimulus from the time required to make the appropriate response following detection. In a test that held detection times constant, Flykt (2006) found that humans respond more rapidly to threatening than nonthreatening stimuli. While this does not change the rationale for the current experiments, which study location memory for threatening stimuli, it does affect how the results from the learning phases are interpreted. Flykt argued that faster responding to threatening stimuli implies that humans are able to rapidly avoid or escape the presence of threatening stimuli.

Detection of and attention to threatening faces. Facial expressions are important in social interactions (Beall \& Herbert, 2008; Frith, 2009). They communicate emotions between people and therefore guide interactions. This includes the ability to detect anger or fear in the expression of another, two expressions that indicate the presence of threat in the environment. Based on this, researchers have suggested that humans should be especially good at detecting threat by observing the expressions of others because of the survival benefits such an ability would infer (Öhman, Lundqvist, \& Esteves, 2001).

The evolutionary psychological hypothesis of threat detection is similar to the one given for memory above, and is generally laid out as follows. In the past humans were exposed to a wide array of stimuli: some neutral with no consequences for survival, some positive and useful for survival, and some negative and threatening to survival. Humans 
differed in the amount of time they needed to detect the presence of these various stimuli. Humans who detected threatening stimuli more rapidly than nonthreatening stimuli were more likely to survive than humans who detected threatening and nonthreatening stimuli equally rapidly, because they could more quickly execute evasive or defensive behaviors. Since those individuals were more likely to survive, they were also more likely to reproduce and pass on their traits, including the ability to rapidly detect threatening stimuli. As the descendants of those humans who could rapidly detect threatening stimuli, modern humans should also possess the ability to detect threatening stimuli more rapidly than nonthreatening stimuli.

Research in this area began with a paper showing that an angry face was detected more rapidly in a crowd of happy faces than a happy face was in a crowd of angry faces (Hansen \& Hansen, 1988). Although this original research was criticized for methodological reasons (Purcell, Stewart, \& Skov, 1996), further research confirmed the finding that threatening faces are detected more rapidly than nonthreatening faces (Becker \& Detweiler-Bedell, 2009; Eastwood, Smilek, \& Merikle, 2001; Fenske \& Eastwood, 2003; LoBue, 2009; Öhman, Lundqvist, \& Esteves, 2001).

The above studies support the idea that threatening faces may be detected more rapidly than nonthreatening faces in the natural environment. They have even provided evidence that these stimuli are avoided more quickly than nonthreatening stimuli (Becker \& Detweiler-Bedell, 2009), which is important because the trait of rapid detection of threatening stimuli can only be beneficial if it actually leads to quick action, potentially including avoidance or escape from the threatening stimulus itself. In addition to rapid 
attention to threatening faces, research has shown that other threatening stimuli are also attended to more quickly than nonthreatening stimuli.

Detection of and attention to other threats. A key finding that sparked interest in attention to threatening stimuli other than faces was reported by Öhman, Flykt, and Esteves (2001). These authors showed that stimuli which posed a threat to humans in the ancestral environment (snakes and spiders) were detected faster in an array of stimuli that posed no threat in the ancestral environment (flowers and mushrooms ${ }^{4}$ ) than when nonthreatening stimuli were presented in an array of threatening stimuli. The authors also found that the search slope remained the same for threatening, but not nonthreatening, targets regardless of the size of the array (either $2 \times 2$ or $3 \times 3$ grids). Based on these results the authors claimed that humans have been selected to rapidly detect stimuli which posed a threat to them in the ancestral environment.

The finding that ancestral threatening stimuli are detected more rapidly than nonthreatening stimuli has been replicated multiple times since Öhman, Flykt, and Esteves (2001), and is now well established (LoBue \& DeLoache, 2010). However, the claim that this is due to humans being naturally selected to rapidly attend to stimuli that were threatening in the ancestral past has been challenged (see LoBue, Rakison, \& DeLoache, 2010, for a summary of these arguments). Other researchers have argued that learning that certain stimuli are threatening is just as likely to account for the results as a specific adaptation for rapid detection of ancestrally threatening stimuli.

In support of this challenge, Brosch and Sharma (2005) performed a study similar to that of Öhman, Flykt, and Esteves (2001), except with the addition of modern 
threatening (guns and syringes) and nonthreatening (cups and mobile phones) stimuli. They found that, while ancestral threatening stimuli were again detected more rapidly than ancestral nonthreatening stimuli, modern threatening stimuli were also detected more rapidly than modern nonthreatening stimuli as well. They also found that the search slopes remained the same for threatening stimuli, regardless of ecological relevance (ancestral or modern) ${ }^{5}$, as the grid size increased from $2 \times 2$ to $3 \times 3$.

Brosch and Sharma's (2005) findings have been replicated by others (e.g., Blanchette, 2006; Fox et al., 2007). In each case, the authors argued that the hypothesis that humans have been selected to rapidly detect stimuli that threatened the survival of our ancestors is too narrow. They proposed instead that humans have been selected to rapidly detect stimuli that they have learned pose a threat to them.

A problem with all of the aforementioned studies is that individual experience with the target stimuli was not accounted for. Therefore, it remains possible that ancestral threatening stimuli were detected more rapidly because of a specific adaptation, rather than because of learning. Subjects could have detected ancestral threatening stimuli more rapidly, even though they had never personally had a negative experience with them. Research by LoBue and colleagues (DeLoache \& LoBue, 2009; LoBue \& DeLoache, 2008, 2011; LoBue, Rakison, \& DeLoache, 2010) has shown that children as young as seven months old detect ancestral threatening stimuli more rapidly than ancestral nonthreatening stimuli. The young age of these children makes it unlikely that they had a personal negative experience with the threatening stimuli. It is also unlikely that they learned of the threat posed by these stimuli via indirect means such as stories or observing others. 
In order to directly address the issue of experience, LoBue (2010a) tested children's detection times for two different sets of modern threatening stimuli. Parents reported that all children had personal negative experiences with some of the stimuli (syringes) but not others (knives). LoBue found that children detected threatening stimuli more rapidly than nonthreatening stimuli only if they had previously had a negative experience with them; that is, only syringes were detected more rapidly than nonthreatening stimuli. Based on these findings, LoBue (2010a) and colleagues (LoBue et al., 2010) concluded that there are multiple pathways for rapid detection of threat: one innate $^{6}$ and one learned.

Relationship between attention and memory. The studies mentioned above focused on whether humans detect threatening stimuli in the environment more rapidly than nonthreatening stimuli. The evidence from these studies support the idea that humans have been adapted to rapidly detect stimuli perceived as threatening, whether they are ancestral or modern. This has led some authors to ask whether this threat advantage extends to memory (Nairne et al., 2012). Given the relationship between attention and memory, there are good reasons to believe that there could be a threat advantage in memory. Stimuli that are the focus of attention are also more likely to be remembered, when memory is assessed by explicit conscious tests (Chun \& TurkBrowne, 2007). Additionally, experience with and memories for certain stimuli influence the rate of attention to those and similar stimuli at later points (Chun \& Turk-Browne). Even more relevant to the current research are the relationships between spatial attention and memory. Evidence suggests that the neural substrates which are activated during spatial attention tasks heavily overlap with those that are activated during spatial memory 
tasks, suggesting that spatial attention and memory may be dependent on each other (Awh \& Jonides, 2001).

Based on these findings, if threatening stimuli are attended to more rapidly, and presumably capture and hold attention more than do nonthreatening stimuli (Koster, Crombez, Van Damme, Verschuere, \& De Houwer, 2004), then there should also be superior memory for these stimuli. The reverse is also implied: Superior memory for threatening stimuli should lead to more rapid detection and recognition of them than nonthreatening stimuli. This is an important point and supports the claim that rapid detection can be due to learning, not necessarily only an innate module for detecting threatening stimuli.

Given the close relationship between attention and memory, it is fair to argue that what is found in research on human attention also warrants study in memory research. The summary provided above clearly shows that humans are faster at detecting threatening stimuli than nonthreatening stimuli. This fosters the expectation that they should also be more likely to remember certain properties of threatening stimuli, possibly including the locations where they were encountered. Past research on other aspects of memory also supports this expectation.

\section{Memory Research}

The conduct of memory research from an adaptationist point of view is not new. Garcia and Koelling (1966) showed that rats learn associations between stomach sickness and taste more readily than they learn associations between stomach sickness and visual or auditory stimuli. The authors explained this finding by arguing that natural selection 
has shaped individuals to learn associations between certain stimuli that have been readily and reliably paired together in the ancestral history of their particular species. This led to a new line of thinking about what certain species, including humans, may be prepared to learn (Seligman, 1971).

Prepared Learning. Prepared learning is the idea that individuals of a particular species are "prepared", or predisposed, by natural selection to quickly learn about certain stimuli and/or associations between stimuli that played an important role in the evolutionary history of that species. In the original paper on this topic, Seligman (1971) argued that phobias in humans typically reflect prepared learning. He argued that most human phobias concern stimuli that could have been threatening in our ancestral environment, such as spiders, snakes, and heights, even though they may not be particularly threatening on a day-to-day basis in the modern world. In contrast, humans typically are not phobic of stimuli that are dangerous in our daily lives, such as guns, knives, or automobiles (LoBue, 2013).

In support of Seligman's (1971) theory, research with humans and nonhuman primates has shown that associations between some stimuli are indeed learned better than others. One example is a pair of studies performed by Cook and Mineka (1989) involving Rhesus monkeys and observational learning. The researchers studied young Rhesus monkeys who had no experience with either of two natural predators, snakes and crocodiles. Rhesus monkeys raised in captivity do not show any fear of these predators when in their presence for the first time. The monkeys in Cook and Mineka's study did not show any fear when presented with toy snakes or crocodiles before the experimental procedure began. The researchers had the subject monkeys watch videos of another 
monkey who reacted fearfully to either one of the predators, a toy snake or a toy crocodile, or to one of two non-harmful stimuli, a flower or a toy rabbit. The researchers found that after observing these videos, the monkeys who viewed the fearful reactions to one of the predators later reacted fearfully when presented with the predator. In contrast, the monkeys who viewed the fearful reaction to the presentation of one of the nonthreatening stimuli did not themselves produce a fear response when presented with either the flower or the toy rabbit.

Cook and Mineka (1989) took this as support for the prepared learning hypothesis. Reptiles have been a consistent predator in the evolutionary history of primates (Isbell, 2006) and Cook and Mineka argued that the monkeys quickly learned to fear the two reptilian predators due to the strong selective pressure on past primates to rapidly learn to fear predatory reptiles. The monkeys did not rapidly learn to fear flowers or rabbits, because no such selection pressure existed to rapidly associate these stimuli with threat.

Research with humans has also supported the prepared learning hypothesis. Most notably, a series of experiments by Öhman and colleagues (reviewed in Öhman \& Mineka, 2001) found support for prepared learning in humans using a variety of different methods, centering on the phenomenon of extinction resistance from classical conditioning. In classical conditioning, the CS evokes the CR after repeated pairings with the UCS. If the CS is then repeatedly presented without the UCS, the CS will produce the CR less and less frequently, until it does not produce the CR at all. This is termed extinction. If the CR continues to persist, in spite of many trials of the CS being presented without the UCS, this is termed extinction resistance. Öhman, Eriksson, and 
Olofsson (1975) found that only a single pairing of threatening stimuli (e.g., a picture of a snake) with a negative UCS (e.g., a shock) was sufficient to produce extinction resistance, while significantly more pairings were necessary for nonthreatening stimuli (e.g., a picture of a flower). Another study found that extinction resistance was stronger for threatening than nonthreatening stimuli after each was paired with a shock UCS (Öhman, Fredrikson, Hugdahl, \& Rimmö, 1976). These studies show that humans are more likely to retain the association between fear and certain stimuli than others, namely stimuli that posed a threat to the survival of our ancestors. This is exactly what would be predicted by Seligman's (1971) prepared learning theory.

As discussed above, there is strong evidence that primates, including humans, are prepared to learn that certain stimuli should be viewed as threatening and should be feared, such as animals who consistently threatened the survival of our ancestors. How does this relate to the idea that location memory should be better for threatening than nonthreatening stimuli? One argument is based on the following logic: Stimuli that threatened the survival of our ancestors are more likely to be feared or cause fear responses; these stimuli can then be said to be viewed as emotional stimuli; emotional stimuli may be remembered better than non-emotional stimuli. The question of whether emotional stimuli are remembered better than non-emotional stimuli is an empirical one that has received much attention. This literature is briefly reviewed below.

Memory for emotion. There is a large literature on emotion and memory, including whether emotional stimuli are remembered better than non-emotional stimuli (for a recent review, see Mather \& Sunderland, 2011). Although the research on this topic is vast, it is a difficult issue to study, not least because defining what emotion is can 
be difficult (LeDoux, 1998). Instead of attempting to study discrete emotions, many researchers choose to study the effects of arousal and valence (Cruciani, Berardi, Cabib, \& Conversi, 2011; Wilson-Mendenhall, Barrett, \& Barsalou, 2013).

Arousal could enhance memory, decrease memory, or have no effect. Interestingly, all three outcomes have been reported in various studies (see Mather, 2007). One hypothesis put forward to account for these diverse findings is that arousal enhances memory only for stimuli or details of an event that are deemed important, and win a competition for mental resources due to both top-down and bottom-up selection processes (Mather \& Sunderland, 2011). Because arousing stimuli are frequently deemed to be important in visual scenes, they should be remembered better than non-arousing stimuli.

In addition to research that shows enhanced recognition of arousing stimuli or details of events (Kensinger, Garoff-Eaton, \& Schacter, 2006; Kensinger, 2007), there has also been research on location memory for arousing stimuli, which is particularly relevant to the current dissertation. In a series of studies, Mather and Nesmith (2008) had subjects view both arousing and non-arousing pictures in various locations. The arousing pictures were further broken into negative and positive pictures. The researchers found that the locations of arousing pictures were remembered better than the locations of nonarousing pictures. Furthermore, they found that the locations of negative arousing pictures were remembered better than the locations of positive arousing pictures, even when the stimuli were equally arousing. If threatening stimuli are considered to be both arousing and negative, then Mather and Nesmith's results suggest that the locations of 
threatening stimuli should be remembered better than the locations of nonthreatening stimuli.

Memory for emotional faces. Research has also been performed on memory for emotional faces. Emotional faces signal emotions and give cues about environmental surroundings to observers. Furthermore, they are likely to be arousing and cause emotional reactions in and of themselves (Yang, Cao, Xu, \& Chen, 2012; Yang, Xu, Du, Shi, \& Fang, 2011). Both recognition memory tests and tests of location memory have been performed using these stimuli.

Patel, Girard, and Green (2012) found better recognition memory for emotional than neutral faces. In this study, subjects encoded the emotional expressions of faces either indirectly or directly. Indirect encoding involved identifying the gender of the face. Direct encoding involved identifying the emotional expression of the face. Following indirect encoding, subjects recognized fearful faces better than neutral ones, and, following direct encoding, subjects recognized angry faces better than neutral ones. In both cases, subjects showed superior memory for emotional faces than neutral faces.

Other research has focused on location memory for threatening faces (Bannerman, Temminck, \& Sahraie, 2012). The authors used a modified version of the Corsi Blocks Task to assess spatial memory span. A sequence of blocks in different locations was indicated and subjects had to report the sequence in order as presented. One of the manipulations included using either emotional (happy or angry) faces or neutral faces inside the blocks. The authors found that the presentation of emotional faces within the blocks did not increase subjects' spatial memory span. The authors took 
this as evidence that the locations of emotional stimuli are remembered no better than the locations of non-emotional stimuli. There are two things to keep in mind while evaluating this conclusion. First, the authors tested whether subjects could remember a sequence of locations, rather than an individual location. Second, the authors tested short-term memory only. It may be that different results would occur in long-term memory.

Summarizing the findings regarding memory for emotional stimuli, recognition memory for emotional faces is better than for non-emotional faces (Patel et al., 2012), although location memory may not be (Bannerman et al., 2012). In addition, non-face emotional stimuli are generally remembered better than non-emotional stimuli (Mather \& Sunderland, 2011), including their locations (Mather \& Nesmith, 2008). Taken together, these findings support the SLMTS hypothesis. There are, however, reasons to question this support.

Basic emotions and emotion circuits? One reason we should not take the research reviewed above as direct support for the SLMTS hypothesis is due to the conceptualization of what emotion is. There is a long history of psychological research stretching back to Charles Darwin assuming that all humans possess certain basic emotions, and that these basic emotions can be recognized in universal facial expressions (Ekman, 1992a, 1992b; Lench, Bench, \& Flores, 2013; Lench, Flores, \& Bench, 2011; Shariff \& Tracy, 2011a, 2011b). These basic emotional expressions are commonly used as stimuli in current research, including the research presented above on attention to and memory for emotional faces. 
Despite this long history of belief in universal emotional expression, recent research calls this into question. In fact, much evidence supports the idea that emotional expressions are actually dependent on culture and context, and are not universally recognizable (Barrett, 2011; Barrett, Mesquita, \& Gendron, 2011; Lindquist, Siegel, Quigley, \& Barrett, 2013). In addition to this, it has been argued that humans may not have discrete emotion circuits in the brain (LeDoux, 1998). In light of this, it has been argued that research should focus on survival circuits in the brain, rather than emotion circuits $^{7}$ (LeDoux, 1998, 2012). One way to do this in behavioral research is to focus on stimuli that would produce a survival response, rather than a certain emotional response.

The experiments of the current dissertation will follow the arguments above in attempting to elicit and study survival circuits, rather than emotional circuits. Stimuli that threaten survival, whether ancestral or modern, should activate a survival circuit in the brain, because the organism is required to assess the situation, possibly preattentively, and perform some sort of action in response (e.g., freezing, fleeing, fighting). The activation of this survival circuit does not necessarily correspond to any single emotion in particular, but could involve none, one, or several different emotional responses. For example, the presentation of a snake should reliably elicit a defense survival circuit, while it may elicit several different emotions including fear, anger, disgust, and possibly even fascination.

Given possible dissociations between threat responses and emotions, it would be premature to assume that findings with emotional stimuli extend to research with threatening stimuli. In the memory research on emotion described above, the stimuli were not necessarily threatening and may not have activated the same survival circuits as 
threatening stimuli. This is not to say that research on emotional stimuli is irrelevant to the question of whether location memory is better for threatening than nonthreatening stimuli. Instead, research on emotional stimuli should be seen as providing encouraging, but incomplete, support for the SLMTS hypothesis, and as motivating further research using threatening stimuli in particular. Other lines of research do the same, such as those using the adaptive memory paradigm.

Adaptive memory paradigm. The adaptive memory paradigm ${ }^{8}$, as developed by Nairne and colleagues (see Nairne, 2010), has received much attention since the first paper to show enhanced memory for stimuli processed for their survival value (Nairne, Thompson, \& Pandeirada, 2007). Survival processing involves thinking about stimuli in terms of how relevant they are to one's survival. Survival processing is usually induced by having subjects read a passage instructing them to imagine that they are stranded in a savannah grassland and must survive any way they can, such as by foraging for useful items. Subjects are then presented with stimuli and asked to think about how relevant the stimuli are to their survival in the scenario. Much research has shown that processing items in terms of their survival value enhances memory over other well-known memory enhancement techniques, such as processing for pleasantness or self-relevance (Nairne, Pandeirada, \& Thompson, 2008). Nairne and Pandeirada (2008) argue that humans have been selected to remember stimuli that were processed in terms of their survival value.

Many studies by Nairne and colleagues (e.g., Nairne et al., 2007) and other researchers (e.g., Weinstein, Bugg, \& Roediger, 2008) have found enhanced recall or recognition of stimuli following survival processing. In addition, it has been shown that survival processing leads to enhanced location memory for stimuli (Nairne et al., 2012). 
These studies can be taken to support the SLMTS hypothesis. However, there is one important caveat. These findings can only be seen as evidence if threatening stimuli themselves cause individuals to process those stimuli, and perhaps the rest of the environment, in terms of their survival value. Stimuli themselves causing survival processing is different than the presentation of a survival scenario causing survival processing. Although there is some support for the idea that threatening stimuli themselves can cause survival processing (Howe \& Derbish, 2010; see Howe \& Otgaar, 2013), we should be cautious about concluding that this is the case. Research by Nairne and colleagues shows that survival processing is independent of the stimuli that have been studied, a point that Nairne (2010) himself has emphasized.

There exists a challenge to the suggestion that research using the survival processing paradigm supports the SLMTS hypothesis. Recent research has shown that the survival processing advantage can be explained by domain-general mechanisms of memory, such as item-specific and relational processing, self-referential processing, uniqueness and distinctiveness of stimuli and scenarios, and depth of processing (Burns, Hwang, \& Burns, 2011; Howe \& Otgaar, 2013; Kostic, Cleary, \& McFarlan, 2012; Kroneisen \& Erdfelder, 2011). If this is the case, then there is no reason to think that there is any special adaptation or brain module for survival processing. Despite this challenge, research using the adaptive memory paradigm seems to tentatively support the SLMTS hypothesis, and suggests that further research is warranted. Another line of research that provides indirect evidence in support of the hypothesis is research dealing with memory for cheaters following social exchanges. 
Memory for cheaters. One of the earliest studies in the field that is today referred to as evolutionary psychology was performed by Cosmides (1989). Variations of the Wason Selection Task (Wason, 1966) were used to investigate human reasoning in social exchanges. Cosmides concluded that humans have been naturally selected to detect cheaters in social exchanges ${ }^{9}$. This study helped to spark interest in the field of evolutionary psychology in general (Buss, 1991), and selection for a cheater detection module in particular.

Based on this research, other researchers began to argue that humans should not only be able to detect cheating in social exchanges, but should also have superior memory for cheaters (Mealey, Daood, \& Krage, 1996). The reasoning is that humans in the past frequently encountered and traded with other humans. It would be important to remember cheaters in order to avoid exchanging with them again or using more caution while exchanging with them in the future ${ }^{10}$. This is relevant to the current dissertation because cheaters can be considered threatening. Cheaters put individuals at risk of a loss of economic resources, which could lead to potential life-threatening situations such as starvation. Therefore, if memory was found to be better for cheaters than non-cheaters, it could be taken to support the SLMTS hypothesis. An initial experiment did find that the faces of cheaters were remembered better than the faces of non-cheaters (Mealey et al., 1996), but this was true only for individuals described as low-status. The authors argued that this reflects an innate bias to remember cheaters, but especially those who we have the power to punish, namely those of lower status. Although one further experiment replicated these findings, showing that cheaters are both looked at longer and better remembered than non-cheaters (Chiappe, Brown, Dow, Koontz, Rodriguez, \& 
McCulloch, 2004), subsequent research has failed to replicate them (Barclay \& Lalumière, 2006; Mehl \& Buchner, 2008). Other findings in this area have been inconsistent (Barclay, 2008; Bell \& Buchner, 2010; Buchner, Bell, Mehl, \& Musch, 2009).

Recently, Bell and Buchner (2012) reviewed all of the extant studies on this topic. They concluded that, in sum, there is evidence supporting enhanced memory for the faces of cheaters, but this does not mean that there is necessarily any evidence for an evolved mechanism specifically for cheater detection. Instead, they argued that the results point to a general mechanism whereby enhanced memory for cheaters is best explained by humans having better memory for negative stimuli and distinctive or unusual stimuli.

In reference to the current dissertation, the research on memory for cheaters can be seen to provide tentative support for the SLMTS hypothesis. Although the research does not inform us about location memory specifically, there is evidence that cheaters are remembered better than non-cheaters, even if this is due to general, and not specific, mechanisms. Since cheaters can be argued to be threatening stimuli and because threatening stimuli can be considered negative stimuli, the results suggest that something about threatening stimuli should be remembered better, which may include the locations where those stimuli were encountered.

Location memory for threatening stimuli. Although research on location memory for threatening stimuli is now recognized as an important extension of the attention research, little has been published on the subject to date. One of the first experiments that addressed this issue was a test of iconic memory (Kuhbander, Spitzer, \& 
Pekrun, 2011). The researchers presented an array of stimuli around a fixation point. The array remained on the screen for $129 \mathrm{~ms}$. The array contained pictures of positive, threatening, and neutral stimuli. After varying delays following the removal of the array $(14,71,229,500,100 \mathrm{~ms})$ a prompt was given in a location where a picture had previously been. Subjects' task was to identify which picture had previously occupied the location. Location memory decayed at a slower rate for threatening stimuli than nonthreatening (positive or neutral) stimuli. This result provided initial evidence that the locations of threatening stimuli are indeed remembered better than those of nonthreatening stimuli. Although this was only for iconic memory, the result suggests that, because of the slower decay rates for threatening stimuli, they have a greater chance of entering into long-term memory.

Another study tested longer-term location memory for threatening stimuli (Wilson, Darling, \& Sykes, 2011). Subjects played a game of pelmanism, commonly referred to as "memory", with threatening and nonthreatening stimuli. Subjects viewed two $5 \times 4$ grids, each containing 5 pairs of threatening stimuli and 5 pairs of nonthreatening stimuli. One of the grids contained ancestral threatening stimuli, such as predators, while the other contained modern threatening stimuli, such as guns. Subjects' task was to flip over one card and then find its match by flipping over a second card. If a match was made, then the pair was removed from the grid. If a match was not made then an error was recorded, along with the distance in number of pixels between the two chosen cards, and both cards were returned to their original locations in the grid and flipped over as to make the picture once more invisible to the subject. There were two main findings. First, after flipping over the first card, subjects made more errors when 
attempting to find a modern threatening match than when attempting to find an ancestral threatening match. Second, the average error distance was significantly smaller for ancestral threatening stimuli than modern threatening stimuli. The authors argued that these results provide evidence that humans have been selected to remember the locations of ancestral threatening stimuli better than the locations of other stimuli, including stimuli that have only recently become threatening to our survival, such as guns.

While these two experiments provided initial support for the SLMTS hypothesis, there were several limitations. In the Kuhbander et al. (2011) study, the researchers only tested iconic memory. For an adaptation for remembering the locations of threatening stimuli to be useful, memory must last over extended periods of time. Only then can individuals avoid or be cautious around locations where threats were previously encountered. Although a slower decay rate suggests that the locations of threatening stimuli could be remembered better than the locations of nonthreatening stimuli over the long-term, this is not a direct test and should not be taken as clear support for the SLMTS hypothesis.

Kuhbander et al. (2011) also failed to compare memory for ancestral stimuli to memory for modern stimuli. As in the attention research, the difference in memory performance between these two types of stimuli is important for theoretical purposes. Only by comparing location memory for ancestral and modern stimuli can researchers determine whether humans actually have a special adaptation for remembering the locations of ancestral threats, or whether the mechanism is more domain-general in nature. 
The study by Wilson and colleagues (2011) also left several questions

unanswered. The locations of ancestral threatening stimuli were remembered better than the locations of modern threatening stimuli, but the researchers did not make any comparisons to nonthreatening stimuli ${ }^{11}$. In doing so the authors neglected to consider that there are multiple hypotheses that could explain the findings, and instead focused on a particular domain-specific hypothesis that the locations of ancestral threatening stimuli alone should be remembered better because of the recurrent threat they posed to our ancestors.

In addition to the critiques particular to each of the two experiments listed above, there is an additional critique common to both. Both experiments were based on the attention research, showing faster detection of threatening than nonthreatening stimuli. The authors hypothesized that the results should be similar for location memory, but their methods did not allow for a test of both attention and memory. In order to address this limitation, Hackländer and Lyle (2012) developed a method that tested whether there were faster response times to and better memory for the locations of threatening than nonthreatening stimuli and whether this interacted with ecological relevance of the stimuli.

In Hackländer and Lyle’s (2012) Experiment 1, response times to ancestral and modern threatening and nonthreatening stimuli were measured. The method differed from most previous research in the way the background was constructed. In most previous research (but see Lipp, Derakshan, Waters, \& Logies, 2004) the target was presented on a background of stimuli that served as targets for other trials. This method does not allow for a test of location memory for those stimuli, because they will have 
been presented in every location during the experiment. In Hackländer and Lyle's experiment, subjects searched for stimuli with either the traditional background, where targets also serve as background pictures, or on a background of nonthreatening stimuli that did not act as targets on later trials. It was found that threatening stimuli were detected faster than nonthreatening stimuli on both the traditional and new backgrounds, providing that they were presented on ecologically congruent backgrounds, meaning that ancestral nonthreatening and threatening stimuli presented on an ancestral nonthreatening background and modern nonthreatening and threatening stimuli presented on a modern nonthreatening background. This experiment showed that a new method in the search task could be used, which would allow for a later test of location memory for target stimuli.

Experiment 2 utilized this new method, and a search task was followed by a location memory test. Threat level and ecological relevance of stimuli were fully crossed, with ancestral nonthreatening, modern nonthreatening, ancestral threatening, and modern threatening stimuli. Again threatening stimuli were responded to faster than nonthreatening stimuli on ecologically congruent backgrounds. The memory test, however, revealed no differences for location memory between threatening and nonthreatening stimuli.

Due to the discrepant findings in studies testing location memory for threatening stimuli, the current dissertation attempts to provide a clearer and more complete evaluation of the SLMTS hypothesis. The experiments employ a variety of methods to test different aspects of memory. All experiments use stimuli that cross threat level (nonthreatening vs threatening) by ecological relevance (ancestral vs modern), allowing 
for a variety of hypotheses to be tested. All of the experiments test long-term memory, which is important because only memory over extended periods of time would allow for survival and reproductive benefits of an adaptation for superior memory for the locations where threatening stimuli were encountered.

\section{Hypotheses}

In addition to the SLMTS hypothesis, there are other related hypotheses that deserve mention. Several of these hypotheses can be said to be evolutionary hypotheses, as they are purposefully derived from the theory of natural selection. These evolutionary hypotheses all exist on a scale, with some being more domain-general in nature and others being more domain-specific. These hypotheses, including the SLMTS hypothesis, are all compared to the null hypothesis. These hypotheses are summarized in Table 1.

Null hypothesis. The null hypothesis in the current study is that no differences in location memory performance will exist as a function of threat level, ecological relevance, or an interaction between the two. While null hypotheses are generally set up in order to be rejected, strong support would be lent to this hypothesis if results from all three experiments failed to find any evidence of a memorial advantage for the locations of threatening stimuli or the locations of ancestral stimuli.

Specific stimuli. The specific stimuli hypothesis states that humans should respond to stimuli faster and remember their locations better if they were recurrent ancestral threats. According to this hypothesis, faster responding and better memory are the product of specific modules in the brain that are activated by visual representations of 
these stimuli. It is important to note that activation of these modules is elicited by visually detecting the whole stimulus, not a specific feature of the stimulus.

Isbell (2006) argues that snakes have acted as a strong selection pressure on catarrhines, or Old World monkeys and apes, including humans. Isbell contends that our visual and attention systems have been designed in direct response to threats posed by snakes, and venomous snakes in particular ${ }^{12}$. In support of her ideas, research has found that snakes are detected faster than other, nonthreatening stimuli (LoBue \& DeLoache, 2008).

In further support of the specific stimuli hypothesis, Rakison and Derringer (2008) performed a series of experiments testing 5-month old infants' looking times to various stimuli. The authors found that infants consistently looked longer at schematic spiders whose features represented normal spiders than schematic spiders whose features were reconfigured or completely scrambled. They also found that looking times towards schematic flowers did not differ depending on whether the features represented normal, reconfigured, or completely scrambled flowers. The authors argued that these results support the notion that humans possess an innate module for spider detection. This argument is in line with research by LoBue (2010b), which showed that spiders are detected faster than other, nonthreatening stimuli, even when those stimuli were cockroaches, whose gross features resemble those of spiders.

In addition to having a module for detecting snakes and spiders, Penkunas and Coss (2013) argue that humans also possess an innate module for detecting felid, or cat, predators. These authors found that felid predators were detected faster than 
nonthreatening control stimuli (specifically, antelope) by both children and adults. While Penkunas and Cross argued that there is a brain module for detecting felid predators, their study only used lions as exemplars of ancestral threatening stimuli. Due to this, this dissertation will assume that there is, potentially, a brain module dealing specifically with lions, not other felid predators.

Based on the detection research listed above, the specific stimuli hypothesis states that the locations of specific ancestral threats will be remembered better than the locations of other stimuli, including both nonthreatening stimuli and threatening stimuli that were not recurring threats throughout the course of human evolution. The latter would include modern threatening stimuli, such as guns, and certain ancestral threatening stimuli, such as sharks. In relation to the current research, the specific stimuli hypothesis predicts that the locations of snakes, spiders, and lions will be responded to faster and better remembered than the locations of all other stimuli.

Specific features. There is an alternative to the specific stimuli hypothesis that focuses on specific features of stimuli, rather than stimuli as whole objects. This alternative, which will be called the specific features hypothesis, states that certain stimuli that were recurrent threats to humans throughout evolution possessed distinct features and the human brain has been adapted to detect these specific features, rather than the stimuli as wholes.

To date, this hypothesis has only been developed in relation to snakes. Research suggests that the sinusoidal shape associated with snakes is a feature that humans have been selected to detect. Masataka, Hayakawa, and Kawai (2010) performed a variation 
of the typical display search task with snakes as target stimuli among distractors, which were all flowers. The snakes were either in a resting position or in a striking position, which typically involved them being coiled with their heads reared back preparing to lunge out with their jaws. Subjects detected the snakes in the striking posture significantly faster than they detected the snakes in the resting posture.

Another series of experiments (LoBue \& DeLoache, 2011) produced similar results. In one experiment, the researchers found that coiled, non-snake stimuli were detected faster in an array of flowers than flowers were in an array of the coiled stimuli. The same experiment also showed that detection time of coiled non-snake stimuli in an array of snakes was equal to detection time towards snakes in an array of coiled nonsnake stimuli. In still another experiment, the researchers found that the typical rapid detection of snakes in an array of flowers vanished when only pictures of non-coiled snakes were used.

Together, the results from Masataka et al. (2010) and LoBue and DeLoach (2011) provide support for the idea that humans detect snakes faster than other stimuli due to a specific feature, namely their coiled shape. The experiments of the current dissertation do not vary the shape of the snake stimuli used ${ }^{13}$, and therefore a direct test of whether the locations of snakes are remembered better than the locations of other stimuli based on specific features cannot be performed. However, since the specific feature hypothesis only relates to snakes, this hypothesis leads to the prediction that, among all stimuli tested, snakes should be responded to most rapidly and their locations should be best remembered. 
Ancestral threatening stimuli. A generalized version of the specific stimuli hypothesis is that all stimuli that were threatening in the ancestral past should be detected faster and remembered better (Wilson et al., 2011). This hypothesis assumes that all ancestral threatening stimuli share certain features which allow them to be identified as threatening either preattentively or very early in attentional processing. What those features are or could be has not been studied or hypothesized. The ancestral threatening stimuli hypothesis would receive support if the locations of only ancestral threatening stimuli, and not modern threatening stimuli, were remembered better than the locations of nonthreatening stimuli. This result could not readily be taken to support the specific stimuli hypothesis, because not all of the ancestral threatening stimuli used in the current study exerted a strong selection pressure on humans throughout the course of their evolution ${ }^{14}$.

Ancestral stimuli. The ancestral stimuli hypothesis is that the locations of all ancestral stimuli, whether threatening or not, should be detected faster and remembered better than the locations of modern stimuli. This hypothesis has received some support in the attention literature, with certain studies showing very rapid detection of animals (Cosmides \& Tooby, 2013; Drewes, Trommershäuser, \& Gegenfurtner, 2011; New, Cosmides, \& Tooby, 2007; Lipp et al., 2004).

The adaptationist argument in favor of this hypothesis claims that ancestral stimuli in general were important to our ancestors. Ancestral threatening stimuli needed to be rapidly detected and their locations remembered in order for our ancestors to avoid situations that threatened their survival. Humans needed to detect ancestral nonthreatening stimuli rapidly and remember their locations well in order to either hunt 
them or possibly learn about environmental conditions from them, such as where water could be found. Support for this hypothesis would come in the form of superior location memory for ancestral stimuli in relation to modern stimuli.

Survival processing. All of the hypotheses described above focus on the stimuli that are being processed and their defining features, whether they are ancestral, threatening, ancestral and threatening, or specific ancestral threatening stimuli. The survival processing hypothesis takes the focus away from the stimuli and puts it instead on the processer, or rather on how the stimuli are being processed. This hypothesis has been explained in detail by Nairne et al. (2012) and it predicts that the locations of stimuli that are processed in terms of how relevant they are to survival will be remembered better than the locations of stimuli not processed in terms of survival relevance. Indirect support for this hypothesis would be found if the locations of threatening stimuli were remembered better than the locations of nonthreatening stimuli because it could be argued that threatening stimuli themselves lead to survival processing, although Nairne (2010) does not assume this. More direct evidence would be if location memory is better following learning that encourages focusing on the survival relevance of the material versus learning that does not (see Experiment 2 below).

SLMTS hypothesis. This hypothesis can actually take two separate forms, but is here discussed as one because the experiments of the current dissertation are unable to distinguish between the two. In addition, both forms of this hypothesis predict that location memory for threatening stimuli should be better than for nonthreatening stimuli, because threatening stimuli provide information that is more relevant to the survival of the organism than nonthreatening stimuli. Both forms involve learning, but the first form 
focuses on learning exclusively while the second form involves learning and an innate ability to remember the locations of stimuli that were recurrent threats to our ancestors.

According to the first form of the hypothesis, humans have been selected to gain in general intelligence (Chiappe \& MacDonald, 2005). Chiappe and MacDonald argued that general intelligence involves the ability to make associations between stimuli or causes and effects. They also claimed that much of general intelligence is related to working memory capacity. Working memory capacity is operationally defined as "the number of items that can be recalled during a complex working memory task" (Barrett, Tugade, \& Engle, 2004, p553). DeCaro and Beilock (2010, p51) point out that working memory capacity is commonly conceptualized as executive attention, which "allows memory representations to be maintained in a highly active state in the face of distraction”.

Increases in general intelligence would allow humans to more quickly make associations between stimuli we encounter in the environment. Of particular importance would be associations between stimuli and the presence of fear or possibly the activation of defense responses. Once an association is made, and certain stimuli become learned threats, they receive extra attention because of their relevance to survival and safety. In this way threatening stimuli are detected faster and their locations should be remembered better, because the individual has learned to view them as threatening.

The second form of this hypothesis also involves learning, but not to the exclusion of an innate component. In this form, learning about threat accounts for why certain threatening stimuli are detected faster and their locations remembered better. This is 
especially true of modern threatening stimuli. Other stimuli, however, are detected rapidly and their locations remembered better even in the absence of experience and learning because of the important selective pressures those stimuli placed on our ancestors (LoBue, 2010a; LoBue et al., 2011).

Support for the SLMTS hypothesis would come from finding that location memory is better for threatening than nonthreatening stimuli in one or all of the experiments of this dissertation. The reason that the two forms of this hypothesis cannot be distinguished in the current dissertation is because experience with the stimuli is not taken into account in the current experiments. Although this is theoretically possible, and others have accomplished the feat in attention research (LoBue, 2010a), attempting it with the current subject pool (i.e., college-aged students) is improbable because selfreports about encounters with stimuli are not only unreliable but they would not take into account vicarious learning.

General mnemonic principles (GMP) hypothesis. This final hypothesis is based on what is known about how memory functions in humans, regardless of predictions based on the theory of natural selection. The hypothesis is domain-general and does not require additional hypothesizing about individual circumstances that our ancestors faced. This is not to say that this hypothesis is incompatible with the theory of natural selection. Rather, it holds that our memory system evolved to function based on a select few principles that function well over a wide range of environments and circumstances. Instead of human memory having evolved in relation to specific problems, this hypothesis argues that humans are the ultimate generalists, and that our memory evolved to be able to handle all possible problems of memory, including new 
ones that inevitably present themselves over the course of environmental change through time.

Four mnemonic principles that are particularly important to keep in mind for the current dissertation are 1) stimuli that are viewed as self-relevant and/or goal-relevant are generally remembered better than stimuli that are not (Holbrook, Bost, \& Cave, 2003; Symons \& Johnson, 1997) 2) distinctive or unique stimuli are remembered better than more mundane stimuli (Winograd, 1981) 3) positively and negatively valenced stimuli are remembered better than neutral (non-valenced) stimuli (Hamann, Ely, Grafton, \& Kilts, 1999) and 4) more arousing stimuli are generally remembered better than less arousing stimuli (Mather \& Sunderland, 2011). This is not an exhaustive list of the basic mnemonic principles, but is important when considering the experiments presented below. These principles will be discussed further when considering the results of the experiments. 


\begin{tabular}{|l|l|}
\hline Null hypothesis & $\begin{array}{l}\text { No differences in memory based on any factor, such as threat } \\
\text { level, stimulus ecological relevance, background ecological } \\
\text { relevance, or test location }\end{array}$ \\
\hline Specific stimuli & $\begin{array}{l}\text { The locations of certain stimuli (namely snakes, spiders, and } \\
\text { lions) should be remembered better than the locations of all other } \\
\text { stimuli }\end{array}$ \\
\hline Specific features & $\begin{array}{l}\text { Memory for specific features of stimuli should lead to superior } \\
\text { location memory for those stimuli; this only applies to snakes in } \\
\text { the current dissertation }\end{array}$ \\
\hline $\begin{array}{l}\text { Ancestral } \\
\text { threatening stimuli }\end{array}$ & $\begin{array}{l}\text { Location memory should be better for stimuli that posed } \\
\text { recurrent threats to the survival of our ancestors than other } \\
\text { stimuli that did not }\end{array}$ \\
\hline Ancestral stimuli & $\begin{array}{l}\text { Location memory should be better for all ancestral stimuli than } \\
\text { modern stimuli because ancestral stimuli were important to the } \\
\text { survival of our ancestors, as either predators or prey }\end{array}$ \\
\hline Survival processing & $\begin{array}{l}\text { The locations of stimuli processed in terms of their survival } \\
\text { relevance would be remembered better than the locations of } \\
\text { stimuli not processed in terms of their survival relevance }\end{array}$ \\
\hline SLMTS & $\begin{array}{l}\text { Location memory for threatening stimuli should be better than } \\
\text { location memory for nonthreatening stimuli, regardless of } \\
\text { whether the stimuli were ancestral or modern }\end{array}$ \\
\hline GMP & $\begin{array}{l}\text { Location memory should be best for stimuli that are arousing, } \\
\text { uncommon, and either positively or negatively valenced }\end{array}$ \\
\hline
\end{tabular}

Table 1. Summary of hypotheses concerning location memory in the current experiments.

\section{Introduction to Experiments}

The three experiments in this dissertation have the common goal of determining whether the locations of threatening stimuli are remembered better than the locations of nonthreatening stimuli. If the SLMTS hypothesis is not supported, then the further aim of the dissertation is to determine which of the alternative hypotheses described above is 
most consistent with the results. The three experiments used different methodologies to test the SLMTS hypothesis. While all experiments include two phases (a learning phase followed by a memory phase), they differ in either the method of encoding the material or the type of memory test used. Differences are described in greater detail, along with the rationale for them, in the sections introducing each experiment individually. Although the three experiments of this dissertation use a variety of learning and retrieval tasks, they share many methodological features. 


\section{GENERAL METHOD}

\section{Subjects and Recruitment}

Subjects were undergraduate students at the University of Louisville and participated during either the fall semester of 2012 or the spring semester of 2013. Subjects were recruited using an online experiment management system. Subjects earned credit in psychology courses for participating. All subjects who signed up for the research were allowed to participate for class credit and experience, but only data from subjects aged 18 to 30 were analyzed (see McDaniel, Lyle, Butler, \& Dornburg, 2008). Research was approved by the University of Louisville Institutional Review Board (IRB\# 11.0503).

\section{Materials}

Subjects provided consent before beginning the experiment ${ }^{15}$. The experiment was completed on a computer. Experiments were created using E-Studio version 2.0 (Schneider, Eschmann, \& Zuccolotto, 2002). All monitors and keyboards used were identical.

Sixty-four pictures were used as target stimuli. The set of target pictures was created by fully crossing ecological relevance (ancestral versus modern) and threat level (nonthreatening versus threatening). There were two exemplars of eight different types (e.g., snakes, spiders, lions) from each stimulus category (e.g., ancestral threatening) for a 
total of 64 target stimuli. Nonthreatening stimuli, especially ancestral nonthreatening stimuli, were chosen to match as closely as possible the gross physical properties of the threatening stimuli. For example 'worm' was chosen as a match to 'snake' and 'ant' was chosen as a match to 'spider'. For pictures of all target stimuli see Appendix A.

In addition to the 64 target pictures there were 16 nontarget background pictures. Half were pictures of an ancestral stimulus (trees) and half were of a modern stimulus (buildings). Trees and buildings were chosen because of their similarity in gross physical features (i.e., thin and vertically oriented).

\section{Procedure}

Learning phase. The experiments consisted of three phases: learning, memory and ratings. The experiments differed primarily in the memory phase but Experiment 2 also differed somewhat from Experiments 1 and 3 in the learning phase. Nonetheless, the learning phase was similar across experiments in many respects and was based on the search task used by Öhman, Flykt, \& Esteves (2001).

In the learning phase subjects viewed 128 separate $3 \times 3$ grids. Half of the grids contained nine nontarget background stimuli (either all trees or all buildings). The other half contained a single target picture from one of the four target categories (ancestral nonthreatening, ancestral threatening, modern nonthreatening, modern threatening) and eight nontarget background pictures (either all trees or all buildings). Subjects' task was to press a key if all pictures in the grid were from the same category (e.g., nine trees) and to press a different key if one of the pictures came from a different category (e.g., eight trees and one snake). These were called target-absent and target-present trials, 
respectively. Each grid remained on the screen until the subject responded. The location of each target was constant across subjects, but the order of presentation of the grids varied randomly.

Memory phase. Following the learning phase each subject completed the memory phase, which varied depending on the particular experiment. These phases will be described in more detail in the individual method sections of each experiment.

Rating phase. After the memory phase subjects advanced to the ratings phase. Subjects rated every target stimulus in terms of how arousing, threatening, and positive the stimulus was on Likert scales ranging from one (not arousing/not threatening/very negative) to seven (very arousing/very threatening/very positive). These ratings, which were collected in each of the experiments performed, unless otherwise indicated, allow a check of the assumption that nominally threatening stimuli are actually perceived as threatening and nominally nonthreatening stimuli are perceived as nonthreatening by the subjects. This also allows for correlations between rated threat and response time and memory accuracy for each stimulus. 


\section{EXPERIMENT 1: RECALL VERSUS RECOGNITION}

\section{Introduction}

Hackländer and Lyle (2012) tested subjects' memory for the locations of nonthreatening and threatening stimuli and found no differences. This study, however, used a recall test only. This leaves open the possibility that location memory for threatening stimuli is better than for nonthreatening stimuli when assessed by a recognition test. An argument could be made that this would also be the more likely scenario in a real-life situation.

To reiterate, the adaptationist argument is that superior location memory for threatening stimuli should exist because it would help individuals avoid or use caution around potentially dangerous locations in the future. For example, while out foraging for food, an individual may have an encounter with a threatening animal, such as a snake. Subsequently, before setting out on another foraging expedition, the individual may remember the encounter with the snake and where it happened. This would allow the individual to avoid going near the site of the encounter. This scenario involves recall memory, whereby the individual recalls the encounter with the snake and its location without actually needing to be near the location to serve as a reminder.

Alternatively, we can imagine that the individual does not recall the location of the encounter and follows the same path while out foraging. The individual may not avoid the location altogether, but upon coming near it the individual may be reminded of 
the previous dangerous encounter by the presence of retrieval cues, such as the landscape. The individual then proceeds with heightened caution. This scenario therefore involves recognition memory, as opposed to recall. This could be seen as a more plausible scenario, because altogether avoiding areas where threats were previously encountered could have the effect of seriously limiting foraging areas.

If humans have been selected to have superior recognition memory for threatening stimulus locations, but not superior recall memory, then a recognition test would obviously be needed to reveal support for the SLMTS hypothesis. This argument assumes that recall and recognition differ in some way. When considering the relationship between recall and recognition there are two views that one may adopt. One is that recall and recognition are essentially the same, and rely on the same neural substrate. The only difference is that more cues are available for memory in recognition than recall (Haist, Shimamura, \& Squire, 1992; Tulving \& Watkins, 1973). By this view, it would be hard to argue that findings should change qualitatively when switching the test from recall to recognition. In relation to the current research, it would be hard to see how a recognition test of location memory would produce support for the SLMTS hypothesis when a recall test did not.

On the other hand, recall and recognition could be viewed as separate processes relying on different neural substrates (Craik \& McDowd, 1987; Hirst, Johnson, Phelps, \& Volpe, 1988). A recent brain imaging study supports this view, showing activation of different brain areas depending on whether recall or recognition was being tested (Sadeh, Maril, \& Goshen-Gottstein, 2012). This view allows for the possibility of findings differing qualitatively, depending on whether recall or recognition is tested. 


\section{Method}

Subjects. Seventy-nine subjects (63 females, $M_{\text {age }}=20.16$ years, $S D=2.21$ ) participated. Subjects were recruited and selected according to the procedure described in the General Method section. Subjects were randomly assigned to either the recall ( $n=$ 38) or recognition ( $n=41)$ test condition. A number of subjects were excluded from analyses for the following reasons: over 30 years old $(n=7)$, computer problems $(n=1)$, failure to follow instructions $(n=4)$, and response time or memory accuracy scores more than 2.5 standard deviations from the mean $(n=10)$.

Materials and Procedure. Subjects in both test conditions first completed the learning phase as described in the General Method section above. The learning phase was completed without any knowledge of a later memory phase. Therefore, learning of target locations was incidental. The design of the learning phase allowed for withinsubjects comparisons of response times based on three factors: threat level (nonthreatening versus threatening), ecological relevance of the stimulus (ancestral versus modern), and ecological relevance of the background stimuli (ancestral versus modern). It also allowed for response times to individual stimuli to be compared. A twominute break followed the learning phase, in which subjects were asked to wait for further instructions.

Following the two minutes a screen automatically appeared with instructions for the memory phase, which differed depending on test type. For recall, a target stimulus from the learning phase appeared in the middle of the screen for $500 \mathrm{~ms}$ and then disappeared. A 3x3 grid then appeared with a number in the middle of each cell in the 
grid. The subject's task was to press the key that corresponded to the location where the stimulus appeared in the learning phase. Following each trial subjects rated their confidence in their response on a Likert scale, ranging from one (guessing) to seven (very confident). The key dependent measure in the recall condition was proportion of locations correctly identified.

For recognition, a target stimulus from the learning phase appeared in the middle of the screen for $500 \mathrm{~ms}$ and then disappeared. A $3 \times 3$ grid then appeared on the screen with the borders of one of the nine locations highlighted in red. The subject's task was to press one key if the target stimulus appeared in the highlighted location during the learning phase and another key if the target had not previously appeared there. One exemplar from each stimulus type (e.g., snakes) was presented in the correct location and the other exemplar from each stimulus type was presented in the incorrect location. Following each trial subjects rated their confidence as in the recall condition. The key dependent measures in the recognition condition were false alarms, and $d^{\prime}$, which is an unbiased measure of overall performance.

Directly following the memory phase each subject completed the ratings phase. Subjects in the recall condition only rated how threatening and arousing each stimulus was (see General Method for details). Subjects in the recognition condition also made positivity/negativity ratings (see General Methods for details). Due to a computer error positivity/negativity ratings were not collected for the first twelve subjects in the recognition condition. 


\section{Results}

Learning phase. The dependent variable was response time (RT) for correct responses. Incorrect responses were not analyzed (less than $10 \%$ of responses for each subject). Independent $t$ tests revealed no significant differences in RT between test conditions, $t(77)=.243$, so further analyses collapsed across that variable. On targetabsent trials, a paired $t$ test revealed a significant difference in RT between background types, $t(78)=6.594, p<.001$, whereby tree-only grids $(M=882.99)$ were responded to faster than building-only grids $(M=994.09)$ (see Figure 1).

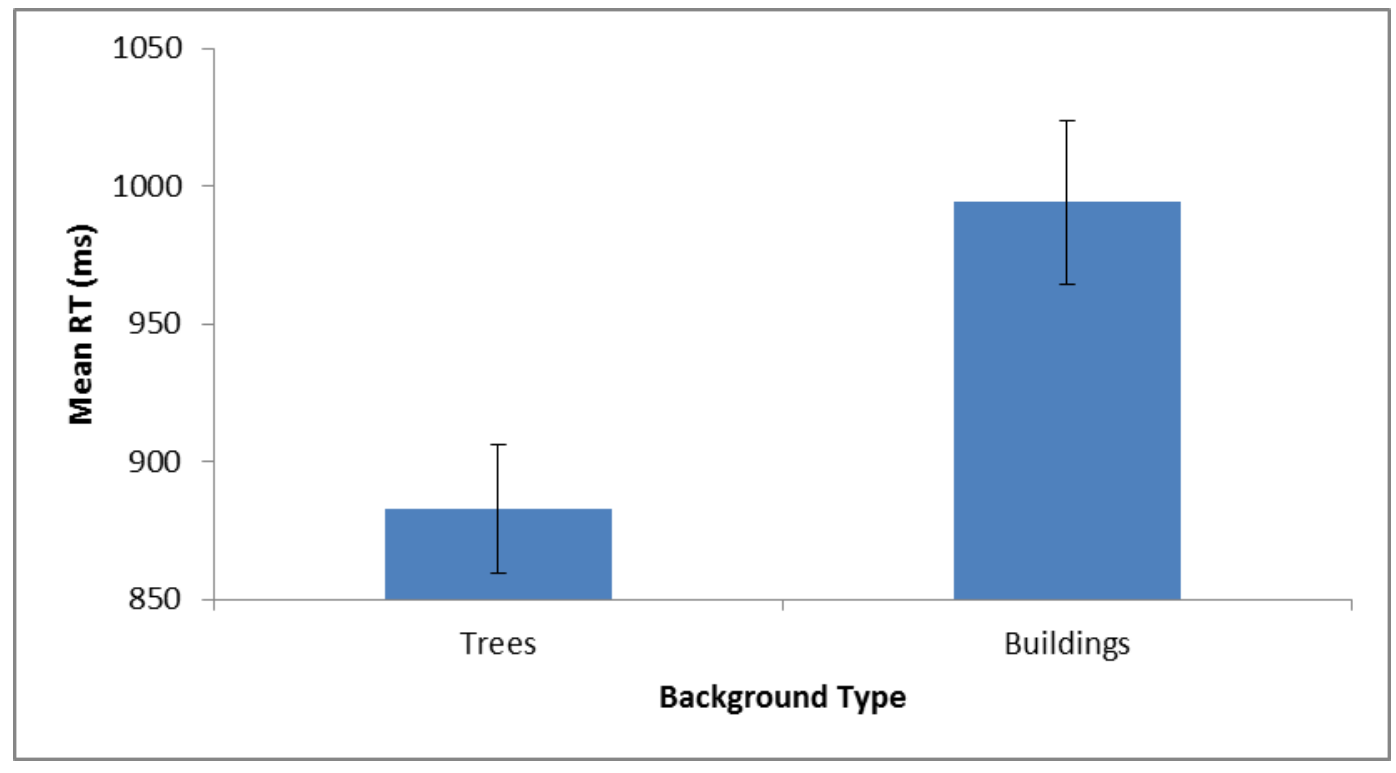

Figure 1. Mean RT on target-absent trials as a function of background type. Error bars on all figures represent $\pm 1 S E M$.

Following these initial analyses, RTs on target-present trials were submitted to a 2 (threat level: nonthreatening or threatening) x2 (stimulus ecological relevance: ancestral or modern) x 2 (background ecological relevance: ancestral or modern) within-subjects ANOVA. There was a significant main effect of threat level, $F(1,77)=4.60, p=.035, \eta^{2}$ 
$=.06$, whereby responses were faster to threatening stimuli $(M=821.65)$ than nonthreatening stimuli $(M=858.96)$ (see Figure 2$)$.

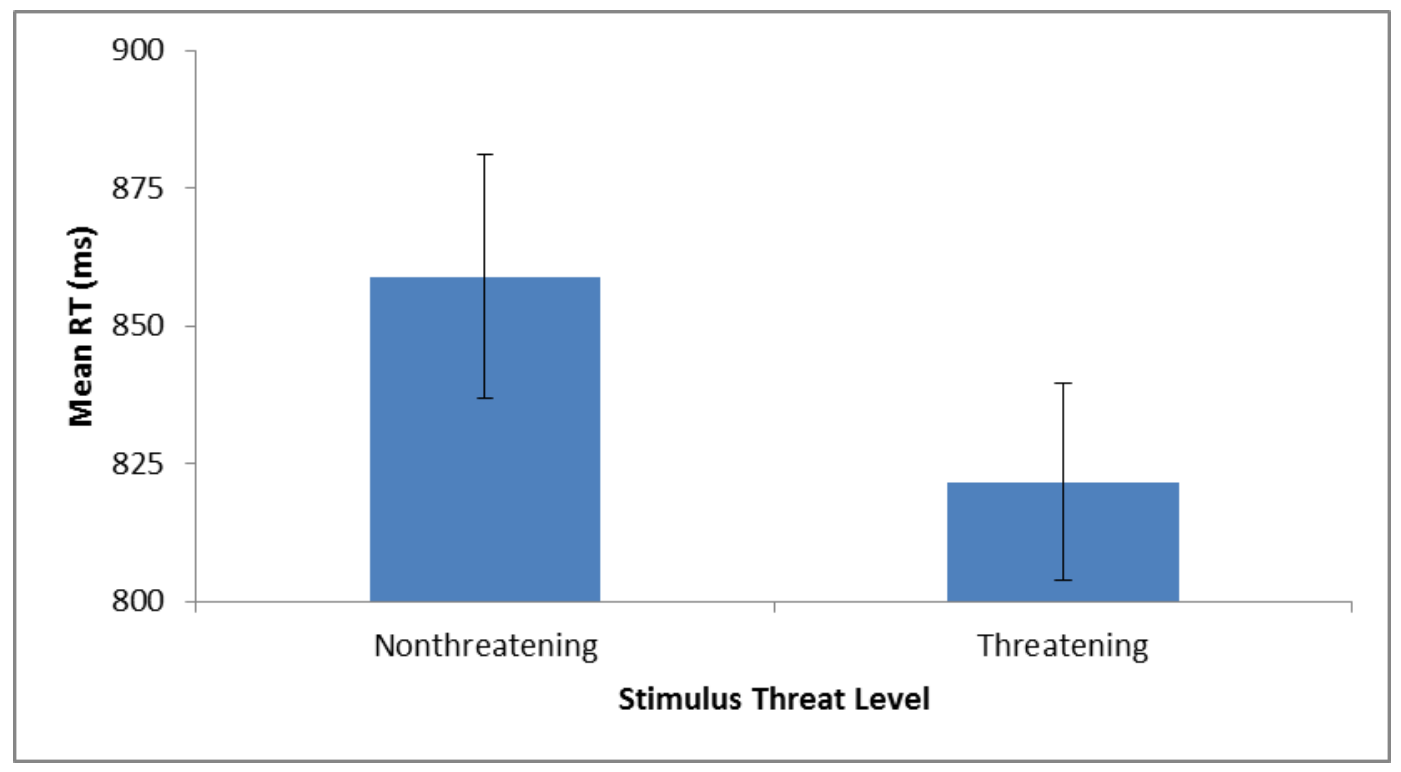

Figure 2. Mean RT on target-present trials as a function of threat level.

There was also a significant interaction between stimulus ecological relevance and background ecological relevance, $F(1,77)=4.21, p=.043, \eta^{2}=.05$, whereby modern stimuli were responded to faster on an ancestral background $(M=815.34)$ than on a modern background $(M=873.14), t(78)=3.43, p=.001$ (see Figure 3 ). RTs to ancestral stimuli did not significantly differ depending on whether presented on an ancestral $(M=$ 839.21) or modern $(M=833.53)$ background, $t(78)=0.23$. 


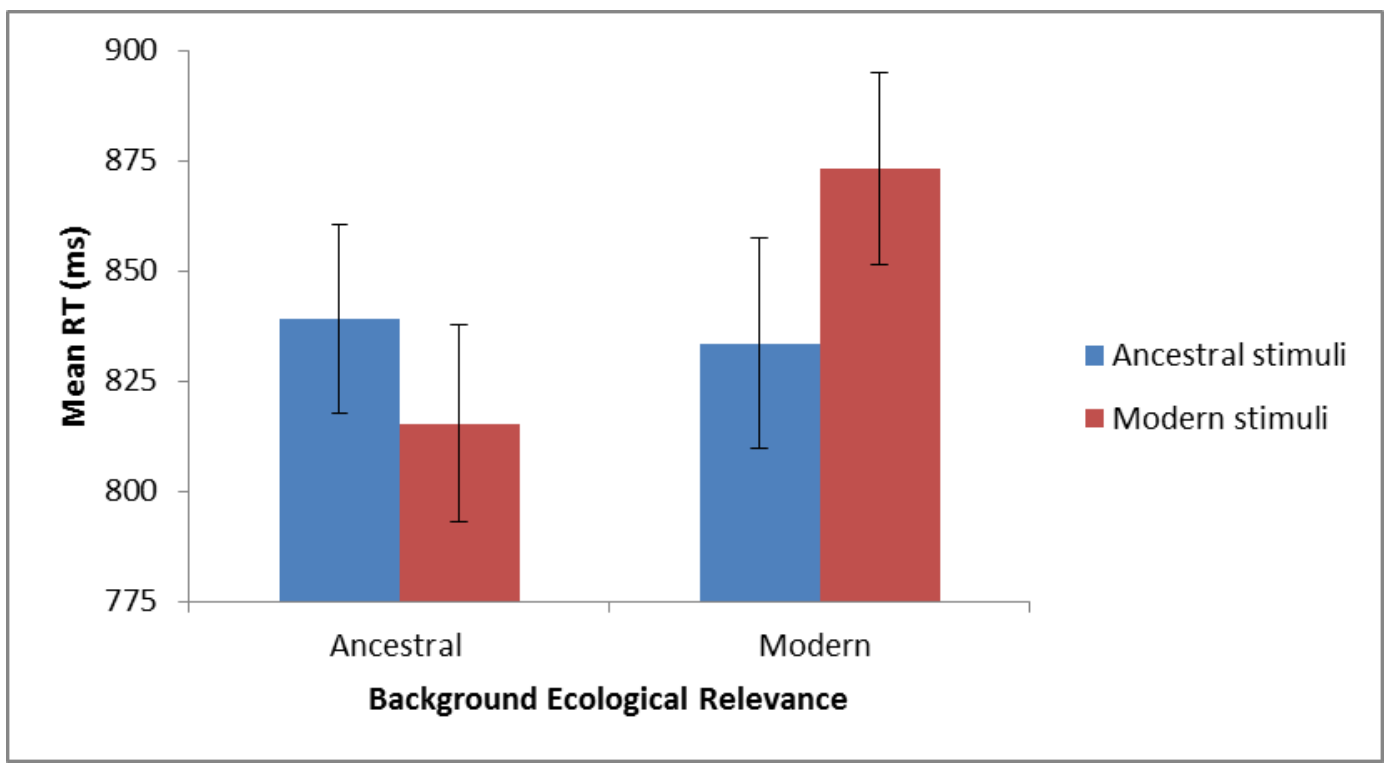

Figure 3. Mean RT on target-present trials as a function of background ecological relevance and stimulus ecological relevance.

\section{Memory phase.}

Recall. The dependent variable was proportion of correct responses, which were submitted to a 2 (threat level: nonthreatening or threatening) x2 (stimulus ecological relevance: ancestral or modern) x 2 (background ecological relevance: ancestral or modern) completely within-subjects ANOVA. There was a significant main effect of threat level, $F(1,37)=5.09, p=.030, \eta^{2}=.12$. Proportion correct was higher for threatening stimuli $(M=.25)$ than for nonthreatening stimuli $(M=.22)$, but the main effect was qualified by a significant interaction with stimulus ecological relevance, $F(1,37)=22.31, p<.001, \eta^{2}=.38($ see Figure 4$)$. Location memory was better for modern threatening stimuli $(M=.27)$ than for modern nonthreatening stimuli $(M=.17)$, $t(37)=5.13, p<.001$. There was no significant difference between ancestral nonthreatening stimuli $(M=.26)$ and ancestral threatening stimuli $(M=.23), t(37)=1.66$, 
neither of which differed from modern threatening stimuli, largest $t(37)=2.02$. Finally, location memory was better for ancestral nonthreatening and ancestral threatening stimuli than modern nonthreatening stimuli, smallest $t(37)=2.93, p=.006$.

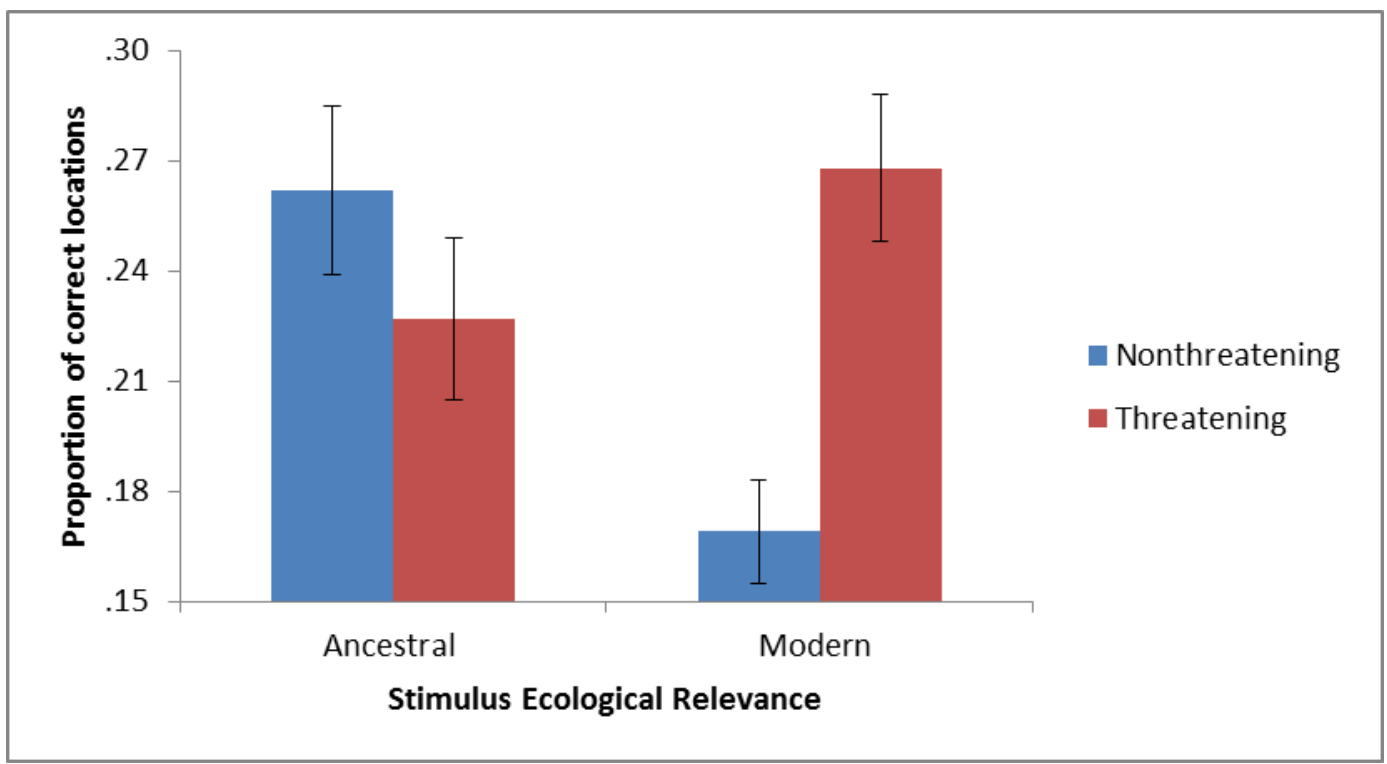

Figure 4. Mean proportion of correct responses as a function of threat level and stimulus ecological relevance.

Recognition. Three 2 (threat level: nonthreatening or threatening) x 2 (stimulus ecological relevance: ancestral or modern) x 2 (background ecological relevance: ancestral or modern) within-subjects ANOVAs were performed. The dependent measures were $d^{\prime}$, proportion hits, and proportion false alarms. For $d^{\prime}$ there was a significant interaction between threat level and stimulus ecological relevance, $F(1,40)=$ $12.26, p=.001, \eta^{2}=.24$, whereby the locations of ancestral nonthreatening stimuli $(M=$ 1.04) were remembered more accurately than the locations of ancestral threatening stimuli $(M=.44), t(40)=2.93, p=.006$, and the locations of modern threatening stimuli 
$(M=.98)$ were remembered more accurately than the locations of modern nonthreatening stimuli $(M=.31)$ stimuli, $t(40)=2.91, p=.006($ see Figure 5$)$.

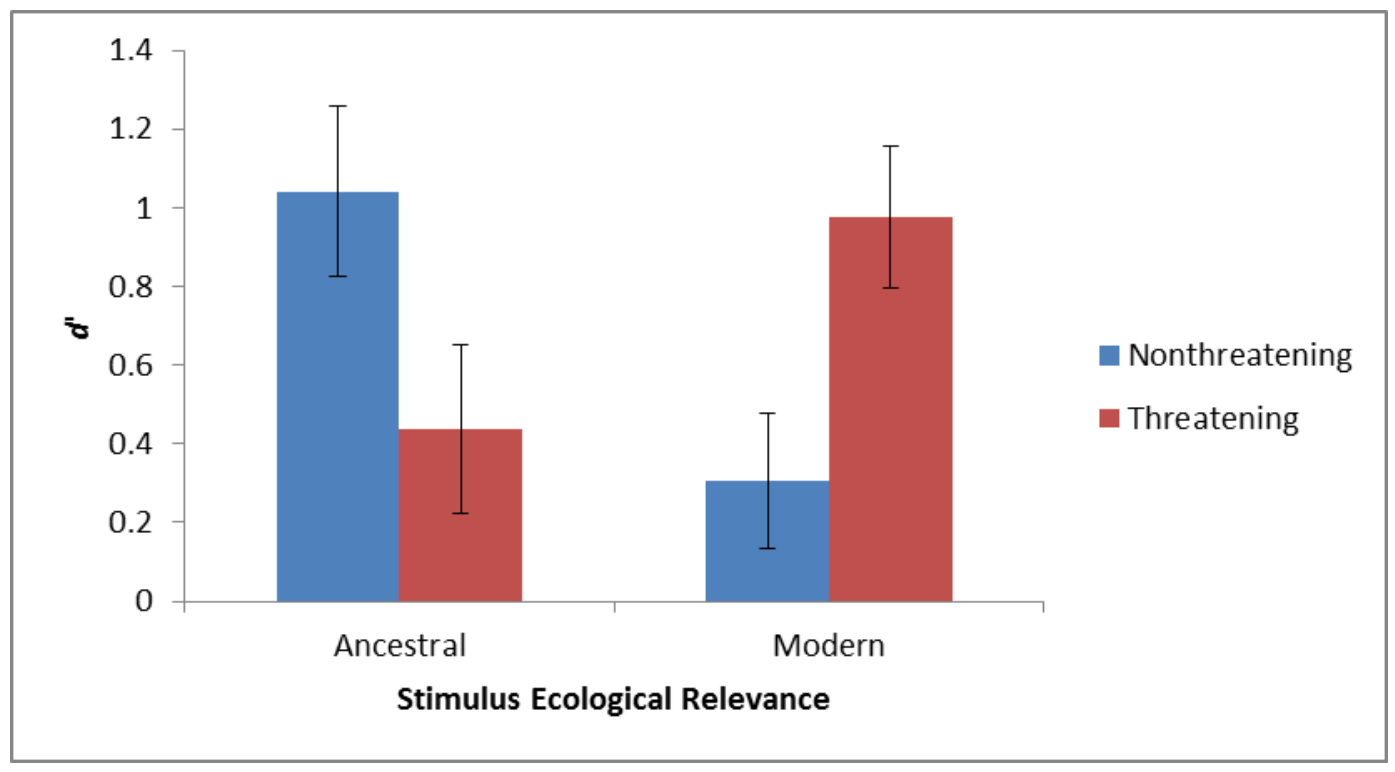

Figure 5. $d$ ' scores as a function of stimulus ecological relevance and threat level.

To determine the dynamics underlying the $d^{\prime}$ interaction, the stimulus ecological relevance by threat level interaction was examined for hits and false alarms. For hits, the interaction was not significant, $F(1,40)=2.47$, but it was significant for false alarms ${ }^{16}$, $F(1,40)=7.70, p=.008, \eta^{2}=.16($ see Figure 6$)$. The false alarm rate was higher for ancestral threatening $(M=.40)$ than ancestral nonthreatening $(M=.28)$ stimuli, $t(40)=$ $3.51, p=.001$, while there was no significant difference between modern nonthreatening $(M=.35)$ and modern threatening $(M=.31)$ stimuli, $t(40)=1.20$. 


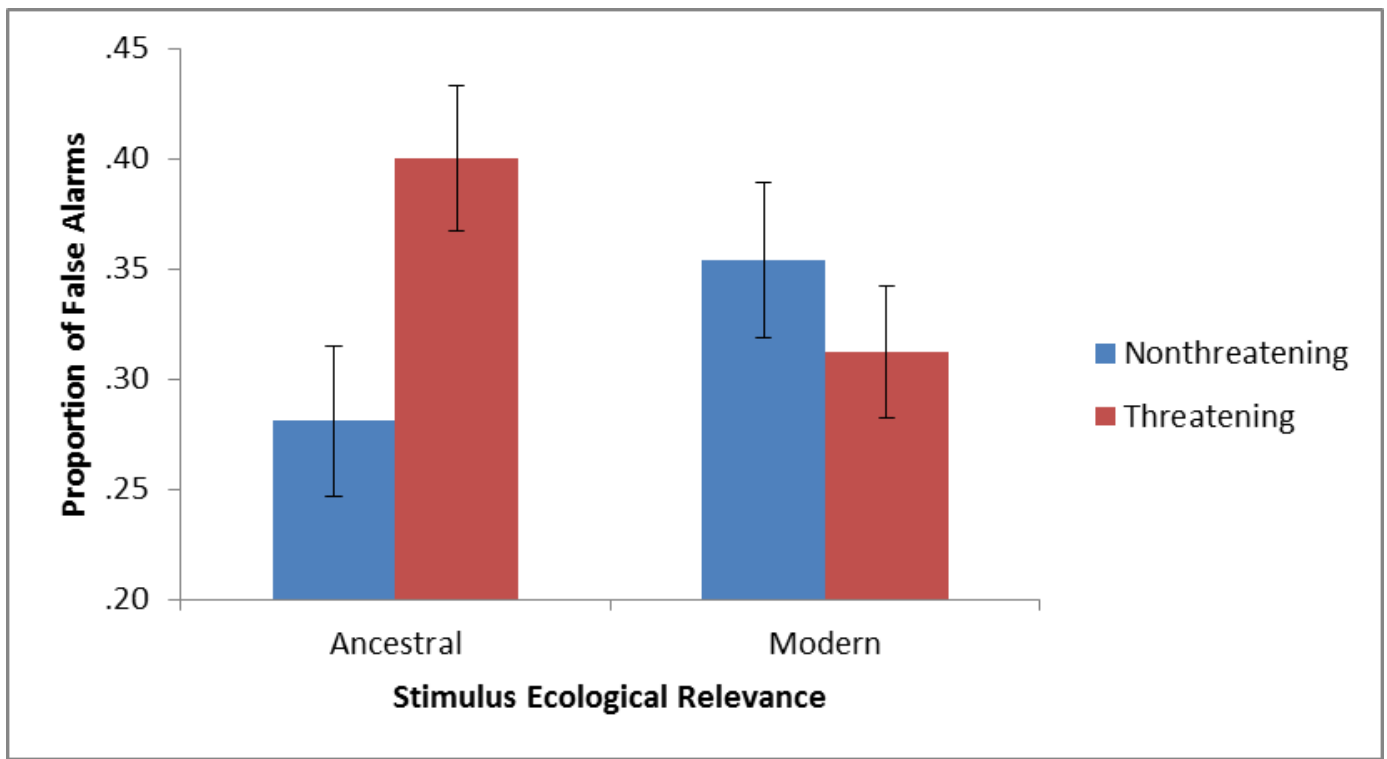

Figure 6. Mean proportion of false alarms as a function of stimulus ecological relevance and threat level.

In addition to the significant interaction between threat level and stimulus ecological relevance in the $d$ ' analysis, there was also a significant interaction between stimulus ecological relevance and background ecological relevance, $F(1,40)=7.36, p=$ $.010, \eta^{2}=.16$ (see Figure 7$)$. Ancestral stimuli $(M=.92)$ that had been seen on ancestral backgrounds were remembered better than modern stimuli $(M=.47)$ seen on the same background type, but the pairwise comparison was not significant, $t(40)=1.73$. The interaction arose because this pattern was reversed for stimuli seen on modern backgrounds: Modern stimuli $(M=.82)$ were remembered better than ancestral stimuli $(M=.56)$. Again, though, this pairwise comparison was not significant, $t(40)=0.96$. 


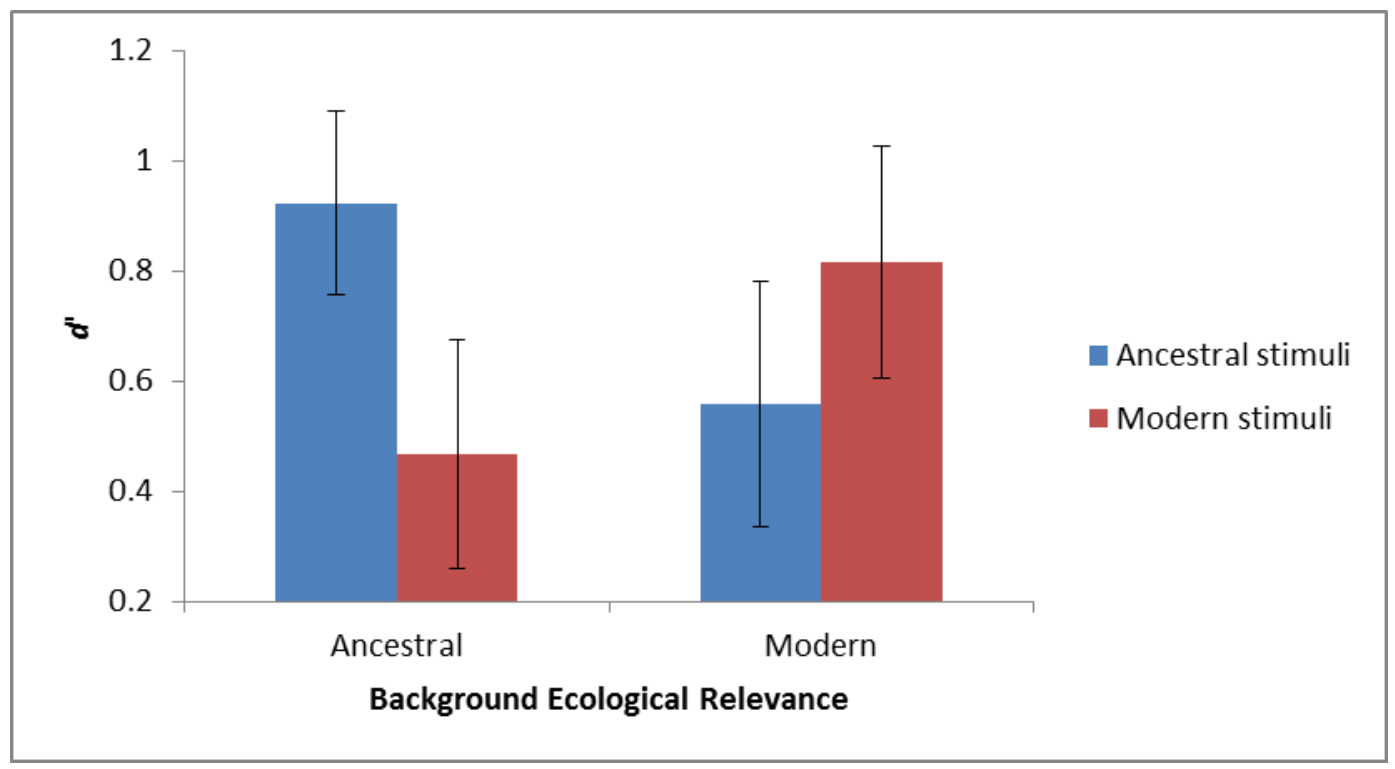

Figure 7. $d$ ' scores as a function of background ecological relevance and stimulus ecological relevance.

A final significant effect in the recognition analyses was the interaction between stimulus ecological relevance and background ecological relevance for hits, $F(1,40)=$ $12.77, p=.001, \eta^{2}=.24$, whereby the hit rate was higher for ancestral $(M=.56)$ than modern $(M=.47)$ stimuli on ancestral backgrounds, $t(40)=2.42, p=.020$, but the hit rate did not significantly differ between modern $(M=.52)$ and ancestral $(M=.46)$ stimuli on modern backgrounds, $t(40)=1.69($ see Figure 8$)$. 


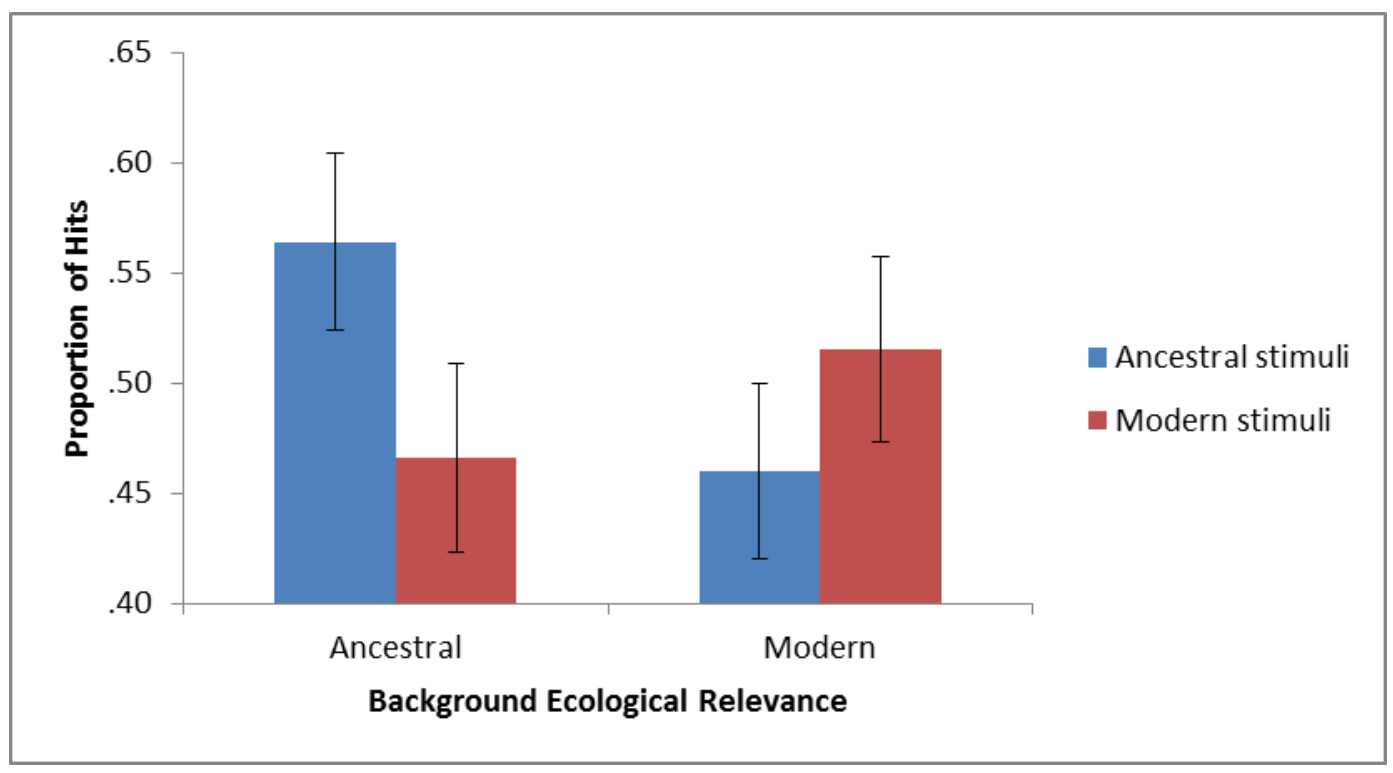

Figure 8. Mean proportion of hits as a function of background ecological relevance and stimulus ecological relevance.

Rating phase. Subjects in both the recall and recognition conditions rated arousal and threat and analyses of these ratings were collapsed across test condition. Only subjects in the recognition test condition rated positivity/negativity. Each type of rating was submitted to a 2 (threat level: nonthreatening or threatening) x2 (stimulus ecological relevance: ancestral or modern) within-subjects ANOVA.

Arousal ratings. There was a main effect of threat level, $F(1,78)=102.07, p<$ $.001, \eta^{2}=.57$, whereby threatening stimuli $(M=4.16)$ were rated as more arousing than nonthreatening stimuli $(M=2.09)$. There was also a main effect of stimulus ecological relevance, $F(1,78)=87.29, p<.001, \eta^{2}=.53$, whereby ancestral stimuli $(M=3.58)$ were rated as more arousing than modern stimuli $(M=2.67)$. There was also a significant interaction between threat level and stimulus ecological relevance, $F(1,78)=54.87, p<$ $.001, \eta^{2}=.41$, whereby the difference in arousal ratings was greater between modern 
nonthreatening and threatening stimuli than between ancestral nonthreatening and threatening stimuli (see Figure 9).

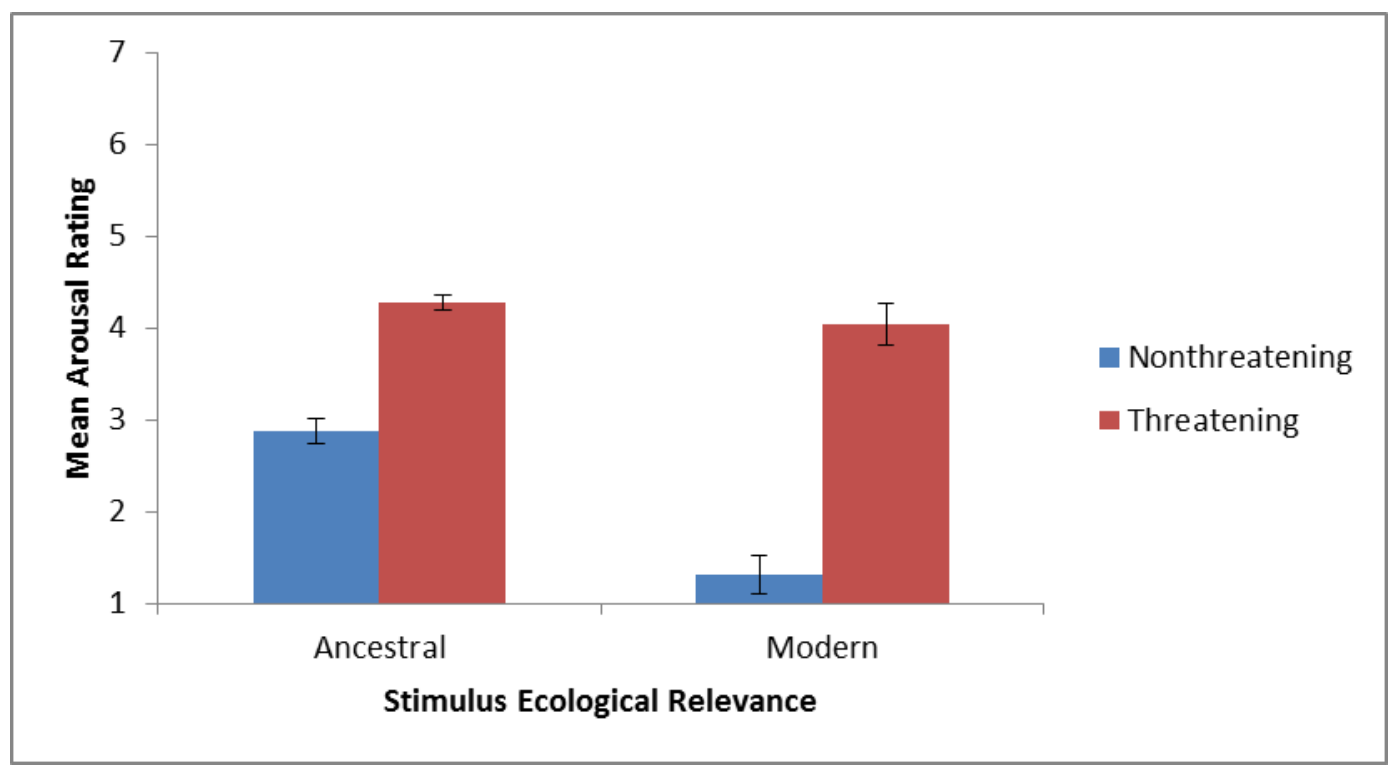

Figure 9. Arousal ratings as a function of stimulus ecological relevance and threat level.

Pairwise comparisons revealed that ancestral threatening stimuli $(M=4.28)$ were rated as significantly more arousing than ancestral nonthreatening stimuli $(M=2.88), t(78)=$ $6.84, p<.001$, and modern threatening stimuli $(M=4.04)$ were rated as significantly more arousing than modern nonthreatening stimuli $(M=1.31), t(78)=11.35, p<.001$. Also, ancestral nonthreatening and ancestral threatening stimuli were rated as significantly more arousing than modern nonthreatening stimuli, smallest $t(78)=11.32, p$ $<.001$.

Threat ratings. There was a main effect of threat level, $F(1,78)=787.51, p<$ $.001, \eta^{2}=.91$, whereby threatening stimuli $(M=5.44)$ were rated as more threatening than nonthreatening stimuli $(M=1.40)$. There was also a main effect of stimulus ecological relevance, $F(1,78)=27.16, p<.001, \eta^{2}=.26$, whereby ancestral stimuli $(M=$ 
3.60) were rated as more threatening than modern stimuli $(M=3.24)$. There was also a significant interaction between threat level and stimulus ecological relevance, $F(1,78)=$ $6.98, p<.010, \eta^{2}=.08$, whereby the difference in threat rating between modern nonthreatening and threatening stimuli was greater than the difference between ancestral nonthreatening and threatening stimuli (see Figure 10).

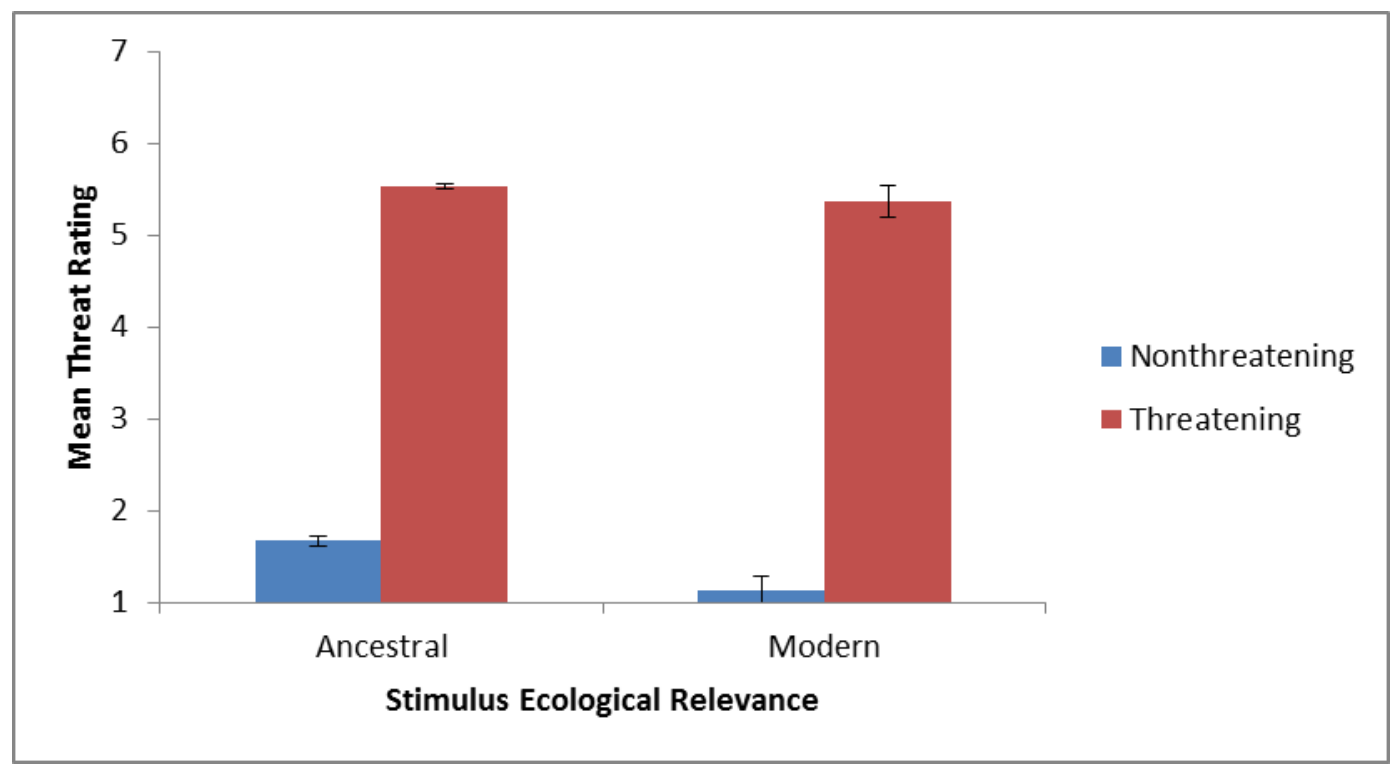

Figure 10. Threat ratings as a function of stimulus ecological relevance and threat level.

Pairwise comparisons revealed that ancestral threatening stimuli $(M=5.53)$ were rated as significantly more threatening than ancestral nonthreatening stimuli $(M=1.67), t(78)=$ $25.13, p<.001$, and modern threatening stimuli $(M=5.36)$ were rated as more threatening than modern nonthreatening stimuli $(M=1.13), t(78)=25.50, p<.001$. Also ancestral nonthreatening and ancestral threatening stimuli were rated as more threatening than modern nonthreatening stimuli, smallest $t(78)=10.47, p<.001$.

Positivity/negativity ratings. There was a main effect of threat level, $F(1,36)=$ $149.64, p<.001, \eta^{2}=.81$, whereby nonthreatening stimuli $(M=4.69)$ were rated as more 
positive than threatening stimuli $(M=2.57)$. There was also a main effect of stimulus ecological relevance, $F(1,36)=5.01, p=.032, \eta^{2}=.12$, whereby modern stimuli $(M=$ 3.50) were rated as more negative than ancestral stimuli $(M=3.77)$. The interaction between threat level and stimulus ecological relevance was not significant, $F<1$ (see Figure 11).

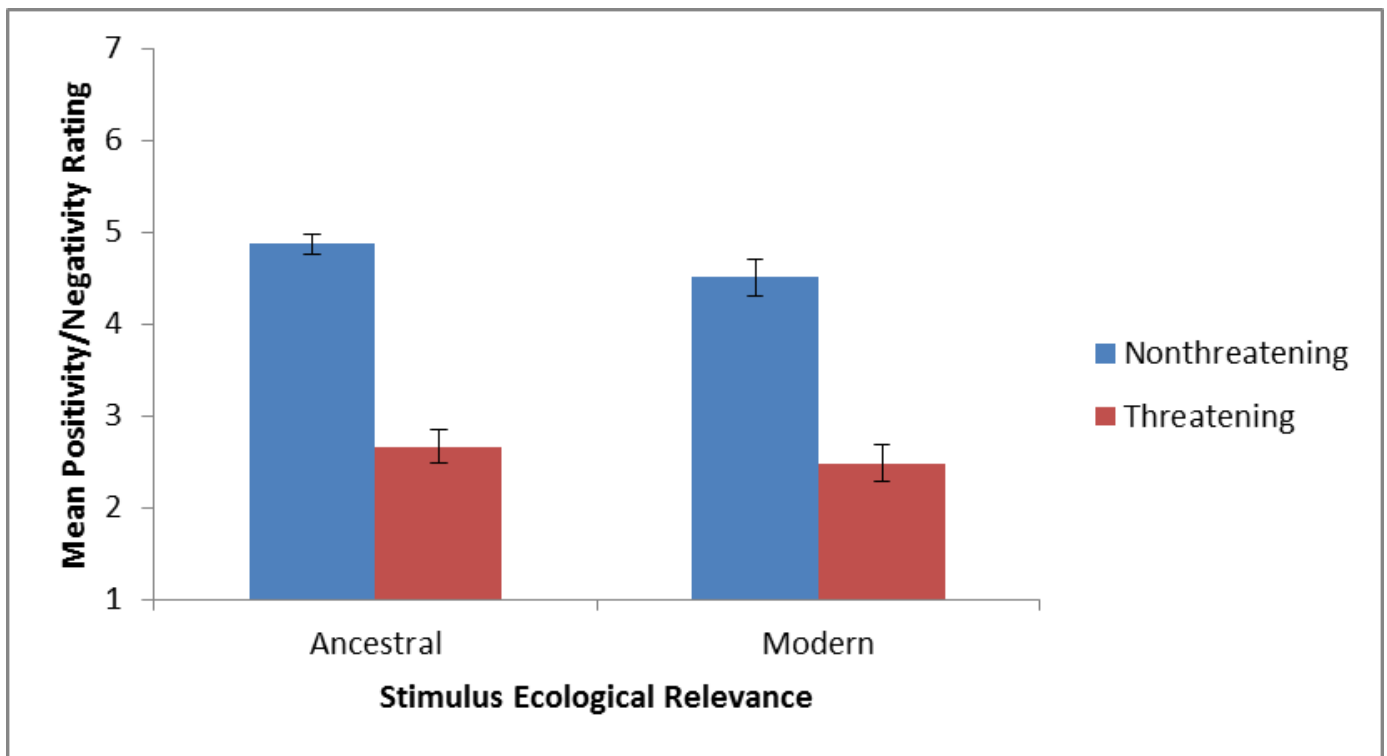

Figure 11. Positivity/negativity ratings as a function of stimulus ecological relevance and threat level.

Pairwise comparisons revealed that ancestral nonthreatening stimuli $(M=4.87)$ were rated as more positive than ancestral threatening stimuli $(M=2.66), t(36)=10.29, p<$ .001 , and modern nonthreatening stimuli $(M=4.51)$ were rated as more positive than modern threatening stimuli $(M=2.48), t(36)=9.47, p<.001$.

Mean positivity/negativity ratings for all stimulus types were submitted to a onesample $t$ test against the scale's neutral point (4). All types differed significantly from neutral (largest $p<.001)$. 


\section{Further analyses.}

Item analyses. $\mathrm{RTs}$ to snakes $(M=841.24)$ and spiders $(M=817.33)$ were slower than RTs to other ancestral threatening stimuli $(M=808.42)$, which does not align with the predictions of the specific stimuli hypothesis. Although RTs to lions $(M=$ 748.85) were numerically faster than RTs to other ancestral threatening stimuli $(M=$ 808.42), the difference was not significant, $t(10)=1.41$. This does not provide strong support for the specific stimuli hypothesis.

Analyses of location memory, like response time, for snake, spider, and lion stimuli did not produce results consistent with the predictions of the specific stimuli hypothesis. In the recall condition, the proportion of correct responses to snakes $(M=$ $.16)$, spiders $(M=.18)$, and lions $(M=.24)$ was lower than the proportion of correct responses to other ancestral threatening stimuli $(M=.25)$. In the recognition condition, the dependent variable for item analyses was the proportion of accurate responses (hits plus correct rejections). Accuracy of responses to snakes $(M=.55)$, spiders $(M=.50)$, and lions $(M=.55)$ was lower than accuracy of responses to other ancestral threatening $\operatorname{stimuli}(M=.57)$.

\section{Discussion}

\section{Learning phase.}

Threatening versus nonthreatening stimuli. There was a significant main effect of threat level, whereby threatening stimuli were responded to faster than nonthreatening stimuli. Ecological relevance of the stimuli did not influence the results, meaning that both ancestral and modern threatening stimuli were responded to faster than ancestral and 
modern nonthreatening stimuli. These results mirror those found in past research (Blanchette, 2006; Brosch \& Sharma, 2005; Fox et al., 2007; LoBue, 2010a) and show that the search paradigm used in the current experiment is comparable to those used in past research, as first suggested by Hackländer and Lyle (2012). Establishing comparability is important because one of the main motivations for the current research is the finding that threatening stimuli are responded to faster than nonthreatening stimuli in search tasks. The experiments of this dissertation slightly alter the search task used in past research to allow for a test of location memory, but Experiment 1 nonetheless yielded comparable findings.

Trees versus buildings. On target-absent trials, tree grids were responded to significantly faster than building grids. This, however, did not translate into a main effect of background ecological relevance on target-present trials: Targets were not located faster on tree grids than on building grids. One interpretation of this finding is that humans have been selected to prefer scenes similar to those lived in during our evolutionary past. It has been shown that humans prefer natural landscapes over modern urban landscapes (Balling \& Falk, 1982; Kaplan \& Herbert, 1987). Furthermore, Falk and Balling (2010) found that humans prefer savannah landscapes over other natural landscapes. If preferred stimuli are detected faster in search tasks, as some research suggests (Chen, Liu, Nakabayashi, 2012), it could be argued that this evolutionary adaptation directly accounts for why the tree grids were responded to faster than the building grids.

A specific adaptation is, however, not required to account for these results. Subjects rated ancestral stimuli more arousing than modern stimuli and the University of 
Louisville is located in an urban setting where buildings are more salient landmarks than trees. When this is coupled with the finding that humans prefer trees to buildings (van den Berg, Hartig, \& Staats, 2007), it supports the conclusion that tree grids were responded to more quickly than building grids because of domain-general mechanisms: tress are more arousing, less common, and better liked than buildings.

Of the two possible explanations for this particular result, the latter appears to be more satisfying because it is more parsimonious than the former. It is more parsimonious because it relies on common domain-general mechanisms that are known to influence human cognition. The other explanation relies on introducing a specific adaptation that must have been selected for over the course of human evolution. Invoking a specific adaptation is rarely parsimonious because: 1) It requires that the adaptation in question provided a strong positive selective pressure for humans over an extended period of time and 2) it is costly in terms of energy and space to designate individual pathways in the brain to the completion of one particular task, such as preferring particular landscapes. Other explanations are also possible. Most notably, buildings and trees differ in various visual features, such as depth of textures, luminosity, and color. It could be that one or more of these visual differences accounts for the faster responses to trees than buildings.

Other findings. The final significant finding from the learning phase was an interaction between stimulus ecological relevance and background ecological relevance. Stimuli were detected fastest when presented on an incongruent background (i.e., an ancestral stimulus on a modern background or a modern stimulus on an ancestral background). This may have been because of the novelty of seeing the targets in 
unfamiliar settings. It is also possible that this was a Type I error, as suggested by the fact that this pattern was not revealed in any of the other experiments.

\section{Memory phase.}

Recall. The recall condition was designed to model a scenario in the natural environment in which an individual wanders through an environment and sees a threatening stimulus. Later, the individual might be able to recall the location of the stimulus and avoid returning to it. This condition was the same as in Hackländer and Lyle (2012), but, in contrast to previous findings, this experiment yielded a main effect of threat level qualified by a significant interaction with stimulus ecological relevance. Location memory for modern nonthreatening stimuli was poor compared to all other stimulus types. This finding does not support the SLMTS hypothesis. Instead, the results seem to most closely conform to the predictions of the GMP hypothesis, which predicts that location memory will be best for stimuli that are, among other things, more arousing and less common.

The arousal ratings showed that both ancestral and modern threatening stimuli were more arousing than nonthreatening stimuli. The ratings also showed that, among nonthreatening stimuli, ancestral ones were significantly more arousing than modern ones. In addition to being least arousing, modern nonthreatening stimuli were the most commonly encountered. Items like spoons and microwaves (two of the exemplars from the modern nonthreatening stimuli) are seen and interacted with more frequently than crocodiles, chainsaws, or worms (exemplars from the ancestral threatening, modern threatening, and ancestral nonthreatening stimuli groups, respectively). It is possible that 
modern nonthreatening stimuli were remembered less well than all other item types because they were less arousing and more commonplace.

This finding is supported by previous research showing enhanced memory for arousing stimuli (Mather \& Sunderland, 2008). Relevant to this dissertation, Schmidt (2012) found that emotional words are remembered better than non-emotional words when the two types of word were presented together in a mixed list. The current experiment used a design similar to a mixed list, in that the four categories of pictures were intermixed. In Experiment 1 of this dissertation and in Schmidt, stimuli that were more arousing and valenced were remembered better than stimuli that were least arousing and non-valenced. It may be that general mnemonic principles explain the findings of Experiment 1 in this dissertation, as well as the superior memory for emotional words in Schmidt.

Recognition. The recognition condition in this experiment was designed to model a possible alternative to the scenario listed in the recall section. In the recognition scenario the individual who has previously encountered a threatening stimulus only remembers the location of that stimulus after wandering through an area near the location of previous encounter. The retrieval cues available remind the individual that a threatening stimulus was previously encountered nearby and the individual proceeds with caution or retreats from the area.

The analysis of false alarms revealed a significant main effect of threat level qualified by an interaction with stimulus ecological relevance. This interaction was driven by an especially high false alarm rate for ancestral threatening stimuli. On the one 
hand this can be taken as evidence of poor memory for the locations of ancestral threatening stimuli. On the other hand this can be seen as evidence that individuals follow the rule of "better safe than sorry" when it comes to remembering the locations of threatening stimuli, especially ancestral threatening stimuli.

The better-safe-than-sorry argument is that falsely remembering the locations where threatening stimuli were encountered can actually be beneficial, or at least less costly, than not remembering them at all. If you falsely remember a predator being in a certain location, and you avoid that location in the future, the only cost would be the loss of a small range of foraging territory. However, if you forget that a predator was in a certain location, and you reenter that location, the cost may be injury or death. Therefore, we may have a tendency to err on the side of caution when remembering the locations where threatening stimuli were encountered.

That humans may err on the side of caution in these situations may be especially true when dealing with ancestral threatening stimuli, because they can be referred to as first-order threats. First-order threats are threats that can act of their own volition, making them threatening in and of themselves. Modern threatening stimuli, on the other hand, are second-order threats. Second-order threats need a second actor in order to make them actually threatening or dangerous.

Howe (2011) argued that false memories can be beneficial depending on the circumstances. One of these circumstances may be when avoiding predators is necessary (Howe \& Derbish, 2010). Given this argument, the high false alarm rate to ancestral nonthreatening stimuli may be taken as evidence that humans have been selected to have 
a lower threshold for "remembering" the occurrence of an ancestral threatening stimulus in a certain location. This, however, is not a necessary conclusion. It may be that humans have a better-safe-than-sorry heuristic, but the stimuli it is applied to are learned throughout the lifetime. Even if we have had negative experiences with modern threatening stimuli, falsely remembering the locations where they were encountered, and then avoiding those locations, would not be beneficial because they are second-order threats.

Another finding relevant to testing the SLMTS hypothesis was an interaction between stimulus ecological relevance and threat level in the analysis of $d$ '. Among ancestral stimuli, the locations of nonthreatening stimuli were more likely to be correctly recognized than those of threatening stimuli, but the reverse was true among modern stimuli (threatening > nonthreatening). These findings are similar to the findings for recall, with the exception that ancestral threatening stimuli were more likely to be recalled, but not more likely to be recognized, than modern nonthreatening stimuli.

$d$ ' is a measure of accuracy that takes both hits and false alarms into account. Therefore the low accuracy score for ancestral threatening stimuli is due to the high false alarm rate for these stimuli. This may be the case, as there were no significant differences for hits between the four stimulus types. The $d^{\prime}$ results, then, would seem to be best explained by the GMP hypothesis. As explained above, modern nonthreatening stimuli were the least arousing and most common stimuli. If the better-safe-than-sorry heuristic causes locations of ancestral threatening stimuli to be falsely remembered at a high rate, then only ancestral nonthreatening stimuli and modern threatening stimuli should have high $d$ ' scores, as was found. 
The GMP hypothesis: recall and recognition. The results of the recall and recognition tests are best explained by general mnemonic principles. Stimuli whose locations were best remembered were those that were more arousing and less common than modern nonthreatening stimuli. The only exception to this was the high false alarm rate for ancestral threatening stimuli, which may be explained by a better-safe-than-sorry heuristic. These results seem to be in opposition to those of Kuhbander et al. (2011) and Wilson et al. (2011) whose results supported the SLMTS hypothesis; this point will be discussed further in the General Discussion.

Other findings. For $d$ ' there was a significant interaction whereby stimuli were most accurately recognized when they had been presented on congruent backgrounds (ancestral stimulus on ancestral background or modern stimulus on modern background) during the learning phase. This interaction was driven by hits, as the same interaction was significant for hits, but not for false alarms. This pattern of results was not predicted, was not found in any of the other experiments, and is not relevant to the main topic of this discussion. Therefore this interaction is not discussed further here. 


\section{EXPERIMENT 2: PROCESS DISSOCIATION PROCEDURE}

\section{Introduction}

Experiment 1 aimed to test the SLMTS hypothesis using recall and recognition tests of location memory. These tested subjects' ability to consciously access previously encoded information, which is sometimes called conscious memory. In contrast to conscious memory is unconscious memory, which is demonstrated when past experiences influence behavior even when they cannot be consciously accessed (Jacoby, 1991; Schacter, 1992, 1996). It is possible that unconscious processes contribute more to location memory for threatening than nonthreatening stimuli. The results of one study indicate that unconscious memory may be greater for threatening than nonthreatening stimuli, at least for highly anxious individuals (Li, Paller, \& Zinbarg, 2008).

To test whether unconscious processes contribute more to location memory of threatening than nonthreatening stimuli, a test of unconscious memory was administered in Experiment 2. The form of the test follows the Process Dissociation Procedure (PDP) as developed by Jacoby (1991), and further refined by Jennings and Jacoby (1993). The PDP has previously been used to test conscious and unconscious contributions to location memory, but not for threatening stimuli (Caldwell \& Masson, 2001; De Goede \& Postma, 2008). 
The PDP involves two different test conditions: inclusion and exclusion. In the inclusion test subjects indicate the location where the stimulus was previously seen. This is similar to a typical recall test and both conscious and unconscious memory may contribute to subjects responding with the actual location. The exclusion test requires subjects to indicate any location other than where the stimulus was previously seen. In this test conscious memory of the actual location should lead the subject to respond with some other location, but unconscious memory of the actual location should lead the subject to inadvertently respond with the actual location. The results of these two tests can be used to create estimates of the conscious and unconscious influences of memory, as explained below in the Method section.

In Experiment 1 and in Hackländer and Lyle (2012), all encoding was incidental, which means that subjects learned the material without knowing that they would be tested on it. It can be argued that the locations of threatening stimuli are not incidentally encoded in natural settings. To illustrate the point we can return to the foraging scenario presented in the introduction to Experiment 1. In this scenario an individual is walking through the environment foraging for food and encounters a threatening stimulus. While foraging, it is possible that the individual is passively taking in information about the environment, but not actively trying to remember anything about the locations where food is found or threats are encountered. This would be simulated by incidental encoding. Alternatively, it may be that the individual is actively encoding location information about threats or signs of threats. A third possible conceptualization is that, when foraging for food or wandering through the environment, humans process their surroundings in terms of their relevance to survival (Nairne, 2010). 
Stimuli were encoded incidentally in Experiment 1 . It could be that only intentional or survival encoding leads to superior location memory for threatening stimuli. Intentional or survival encoding might more accurately reflect the encoding that takes place in a natural setting. There are many examples in memory research of different encoding styles leading to different memory outcomes. For example, associative memory between names and faces is worse in older than younger adults following intentional learning, but not following incidental learning (Naveh-Benjamin, Shing, Kilb, Werkle-Bergner, Lindenberger, \& Li, 2009). Also, survival processing has been shown to produce a different level of memory performance compared to other types of incidental encoding (Nairne et al., 2008). It may be that threat-based differences in location memory also depend on encoding style.

Experiment 2 has two aims. One is to test whether unconscious processes contribute more to location memory for threatening than nonthreatening stimuli. The second aim is to test whether location memory differs depending on encoding style.

\section{Method}

Subjects. Eighty-six subjects ( 71 females, $M_{\text {age }}=20.28$ years, $S D=1.79$ )

participated. Subjects were recruited and selected according to the procedure listed in the General Method section above. Subjects were randomly assigned to one of three encoding conditions: incidental $(n=26)$, intentional $(n=29)$, or survival $(n=31)$. A number of subjects were excluded from analyses for the following reasons: over 30 years old $(n=6)$, computer problems $(n=1)$, failure to follow instructions $(n=16)$, and 
response time or memory accuracy scores more than 2.5 standard deviations from the mean $(n=1)$.

Materials and Procedure. Subjects in all encoding conditions first completed the learning phase as described in the General Method section above but with modifications that depended on condition. For exact instructions, see Appendix B. In the incidental condition, subjects completed the learning phase as described in the General Method section above, without knowledge of a later memory phase. Therefore, learning of target locations was incidental. The intentional condition differed from the incidental condition in that subjects were told that there would be a memory test later and that they should remember the locations where the targets were presented. The survival condition differed from the intentional condition in that subjects were not only made aware of the later memory test, but it was also pointed out to them that some stimuli were more survival-relevant than others, and they were told that remembering the locations of certain stimuli could help them survive in the wild by being able to either find or avoid those stimuli in the future.

In the learning phase in all encoding conditions, the key dependent measure was response time. In addition to the within-subjects comparisons listed above, the design of the learning phase allowed for a between-subjects comparison based on encoding condition: incidental, intentional, or survival. A two-minute break followed the learning phase, in which subjects were asked to wait for further instructions.

After the two-minute break, subjects completed an identical memory phase. The memory phase was based on the procedure used by Caldwell and Masson (2001) and 
included 32 inclusion trials and 32 exclusion trials, which were blocked and counterbalanced between subjects. In the inclusion task the target stimulus was presented in the middle of the screen and subjects had to choose the location on a $3 \times 3$ grid that corresponded to the location where the stimulus was first seen. The inclusion task was identical to the recall test in Experiment 1. In the exclusion task the target stimulus was presented in the middle of the screen and subjects had to choose any location on a $3 \times 3$ grid that did not correspond to the location where the stimulus was first seen.

The proportion of target locations selected during the inclusion and exclusion tasks allowed for estimates of the influence of conscious $(C)$ and unconscious $(U)$ memory. These estimates are determined by the following formulae, where inclusion $(I)$ and exclusion $(E)$ are observed values representing the proportion of target responses given: an accurate response, choosing the location the stimulus was first seen, on inclusion trials and an inaccurate response, choosing the location the stimulus was first seen, on exclusion trials (Caldwell \& Masson, 2001).

$$
\begin{aligned}
& I=C+(1-C) U \\
& E=(1-C) U
\end{aligned}
$$

Simple algebra allows the above formulae to be expressed as:

$$
\begin{aligned}
& C=I-E \\
& U=E /(1-C)
\end{aligned}
$$

For analytical purposes $C$ and $U$ are considered as two separate dependent measures. This allows for two separate mixed-design ANOVAs with the within-subjects 
factors of target threat (nonthreatening or threatening) and target ecological relevance (ancestral or modern) and the between-subjects factor of encoding condition (incidental, intentional, or survival).

On each memory trial subjects were asked to rate their confidence in their response on a Likert scale ranging from one (guessing) to seven (very confident). On inclusion trials subjects rated how confident they were that they had chosen the location where the target was first seen, while on exclusion trials subjects rated how confident they were that they had chosen a location other than where the target was first seen. Following the memory phase subjects completed the ratings phase. Subjects rated how threatening, arousing, and positive/negative each stimulus was, and also rated how survival relevant each stimulus was, ranging from one (not survival relevant) to seven (very survival relevant).

\section{Results}

Learning Phase. The dependent variable was RT for correct responses. Incorrect responses were eliminated from RT data (less than $10 \%$ of the responses for each subject). On target-absent trials, a paired $t$ test revealed a significant difference in RT between background types, $t(85)=5.48, p<.001$, whereby tree-only grids $(M=$ 933.78) were responded to faster than building-only grids $(M=1053.78)$ (see Figure 12$)$. 


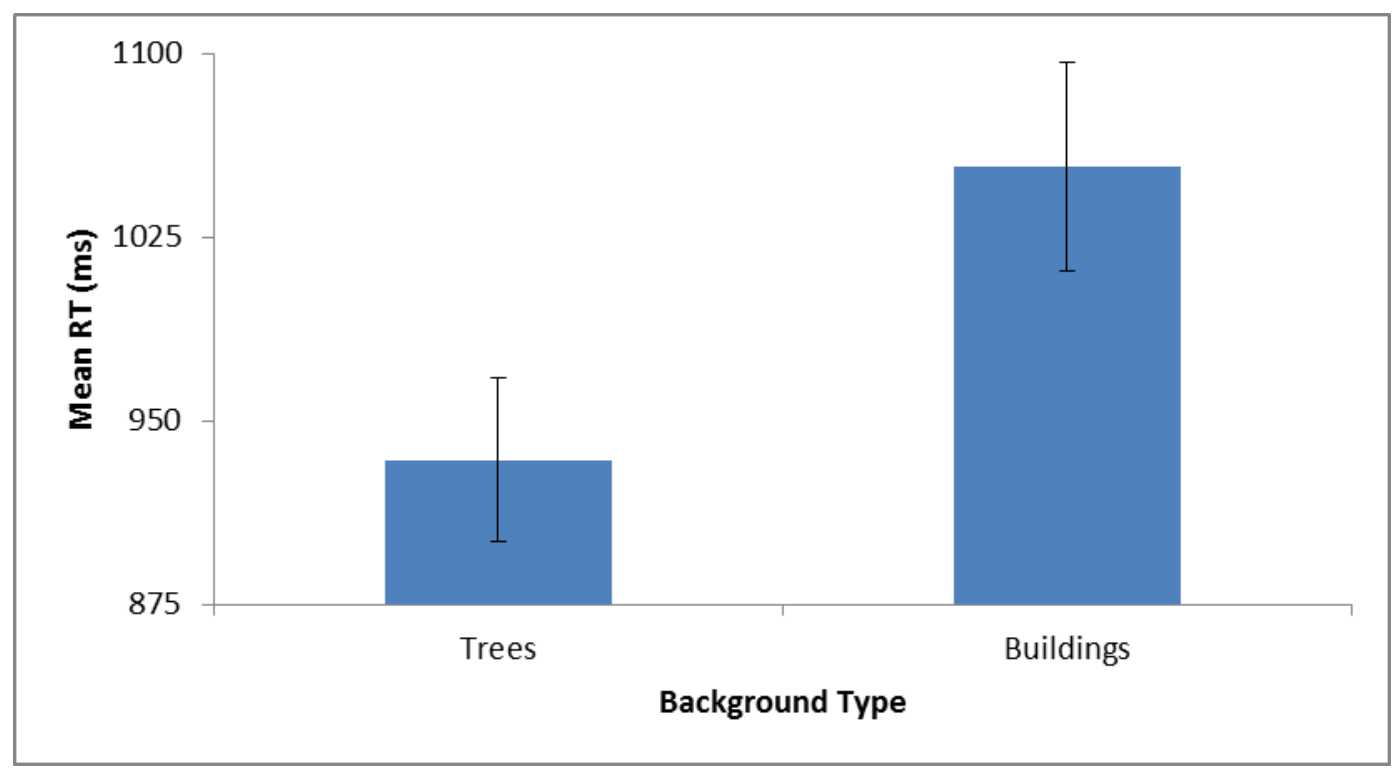

Figure 12. Mean RT on target-absent trials as a function of background type.

Following these initial analyses, RTs on target-present trials were submitted to three 2 (threat level: nonthreatening or threatening) x2 (stimulus ecological relevance: ancestral or modern) x 2 (background ecological relevance: ancestral or modern) x 3 (encoding condition: incidental, intentional, survival) mixed-design ANOVA in which the first three factors were within-subjects and the final factor was between-subjects. This analysis failed to reveal any significant effects. Although neither the main effect of threat level nor the interaction with encoding condition was significant, this was driven by the weak effects in the intentional and survival conditions. When looking at the traditional, incidental condition only, the main effect of threat level was significant, $F(1$, $25)=5.68, p=.025, \eta^{2}=.19$, whereby responses were faster to threatening stimuli $(M=$ 843.52) than nonthreatening stimuli $(M=898.79)$. There was also a significant interaction between threat level and background ecological relevance, $F(1,25)=9.01, p$ $=.006, \eta^{2}=.27$, whereby responses were faster to threatening stimuli $(M=807.15)$ than 
nonthreatening stimuli $(M=933.45)$ on ancestral backgrounds, $t(25)=3.63, p=.001$, but RTs did not significantly differ between nonthreatening stimuli $(M=864.12)$ and threatening stimuli $(M=879.88)$ on modern backgrounds, $t(25)=0.50$, (see Figure 13$)$.

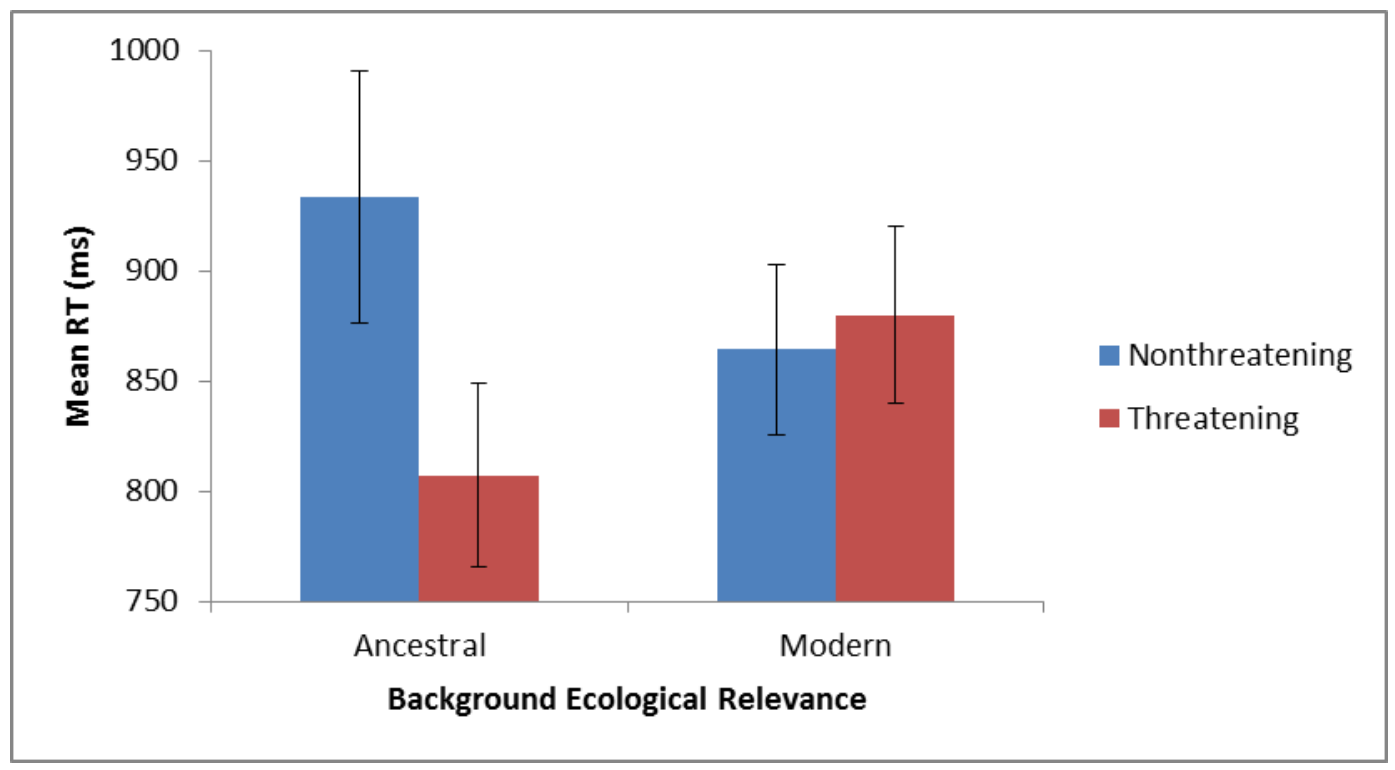

Figure 13. Mean RT as a function of background ecological relevance and threat level in the incidental encoding condition.

Memory Phase. The dependent variable on both the inclusion and exclusion trials was the proportion of target locations selected. Selection of the target's actual location was the nominally correct response on inclusion trials and the nominally incorrect response on exclusion trials. The proportions of target location selections on the two trial types were submitted to two separate 2 (threat level: nonthreatening or threatening) x2 (stimulus ecological relevance: ancestral or modern) x 3 (encoding condition: incidental, intentional, or survival) mixed-design ANOVAs in which the first two factors were within-subjects and the final factor was between-subjects. 
Inclusion trials. There was a main effect of stimulus ecological relevance, $F(1$, $83)=9.55, p=.003, \eta^{2}=.10$, whereby the target locations of ancestral stimuli $(M=.29)$ were chosen more frequently than the target locations of modern stimuli $(M=.24)$. The main effect was qualified by a significant interaction with threat level, $F(1,83)=13.36, p$ $<.001, \eta^{2}=.14$, whereby significantly more target locations were chosen for modern threatening stimuli $(M=.28)$ than modern nonthreatening stimuli $(M=.20), t(85)=3.55$, $p=.001$, while there was no significant difference between the proportion of target locations chosen for ancestral nonthreatening stimuli $(M=.31)$ and ancestral threatening stimuli $(M=.27), t(85)=1.56($ see Figure 14$)$.

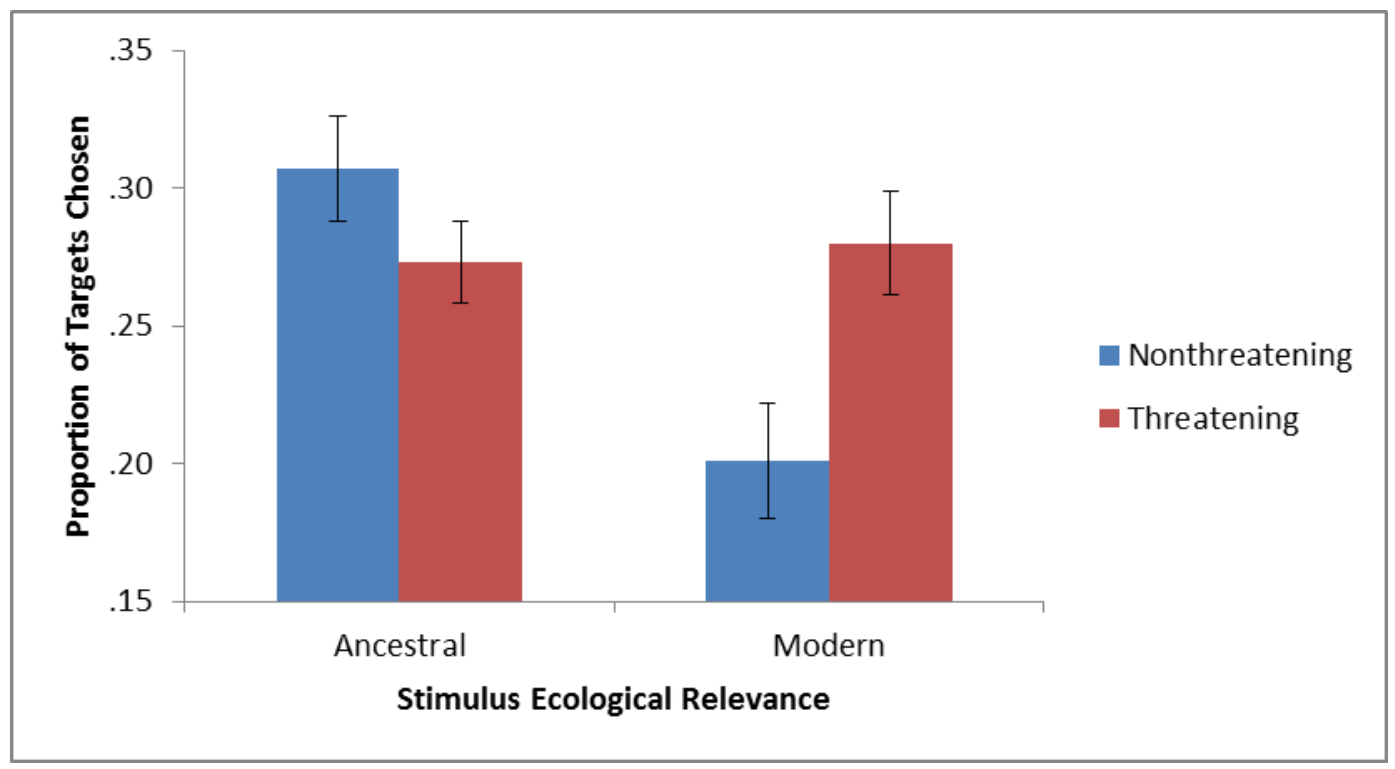

Figure 14. Proportion of target locations chosen on inclusion trials as a function of stimulus ecological relevance and threat level.

Exclusion trials. The only significant effect was the interaction between threat level and stimulus ecological relevance, $F(1,83)=4.51, p=.037, \eta^{2}=.05$ (see Figure 15). For threatening stimuli, more target locations were chosen for modern $(M=.14)$ 
than ancestral $(M=.10)$ stimuli, but the pairwise comparison was not significant, $t(85)=$ 1.86. The interaction arose because this pattern was reversed for nonthreatening stimuli: More target locations were chosen for ancestral $(M=.13)$ than modern $(M=.11)$ stimuli. Again, though, this pairwise comparison was not significant, $t(85)=1.00$.

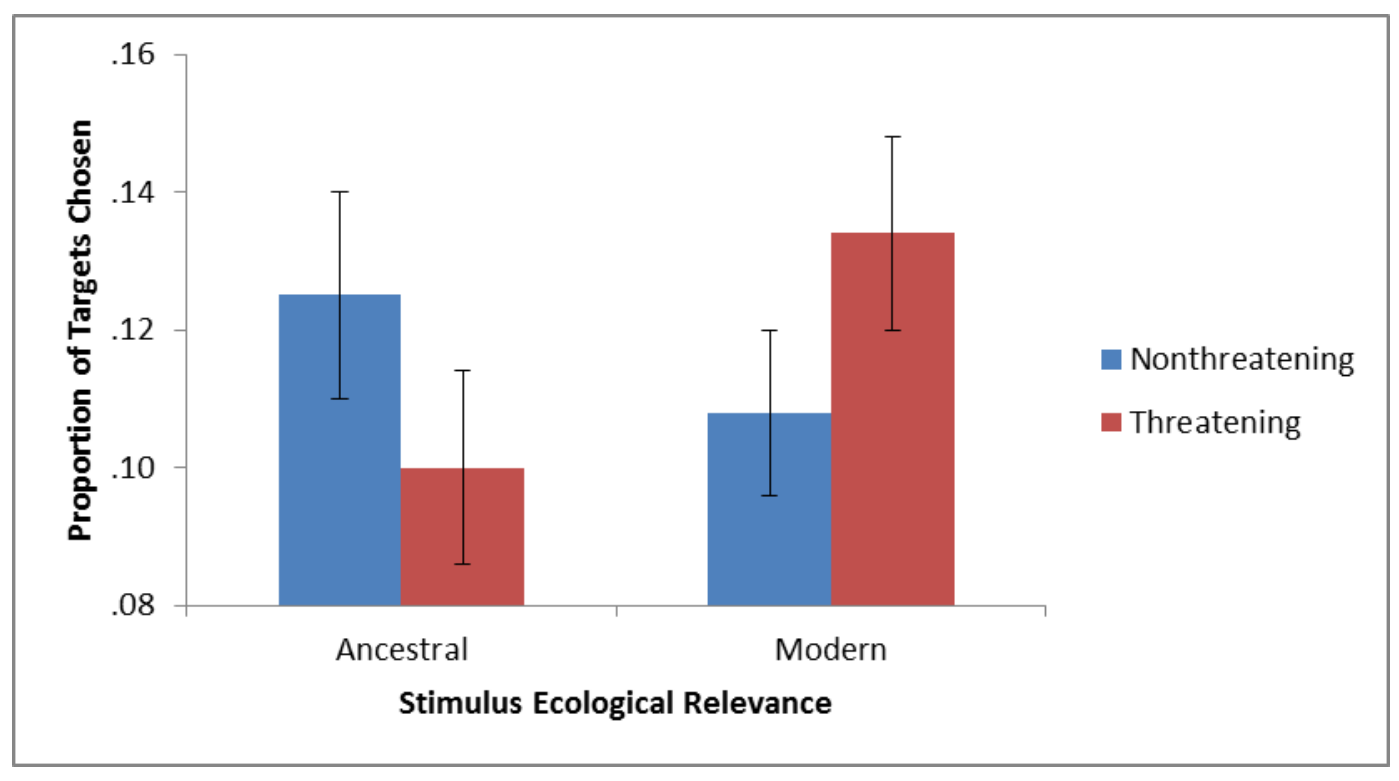

Figure 15. Proportion of target locations chosen on exclusion trials as a function of stimulus ecological relevance and threat level.

Conscious and unconscious scores. Obtaining the observed values of proportion of targets selected on inclusion and exclusion trials allowed for estimations of the influences of conscious and unconscious memory on task performance, as described in the Method section. To determine if conscious and unconscious memory differentially influenced the results depending on the stimulus type and/or encoding style, conscious and unconscious scores were submitted to separate 2 (threat level: nonthreatening or threatening) x 2 (stimulus ecological relevance: ancestral or modern) x 3 (encoding 
condition: incidental, intentional, or survival) mixed-design ANOVAs in which the first two factors were within-subjects and the final factor was between-subjects.

For conscious scores there was a main effect of stimulus ecological relevance, $F(1,83)=6.67, p=.012, \eta^{2}=.07$, whereby the influence of conscious location memory was greater for ancestral stimuli $(M=.18)$ than modern stimuli $(M=.12)$. The main effect was qualified by a marginally significant interaction between threat level and stimulus ecological relevance, $F(1,83)=3.46, p=.066, \eta^{2}=.04$, whereby the influence of conscious memory was greater for threatening $(M=.15)$ than nonthreatening $(M=.09)$ stimuli, $t(85)=2.06, p=.042$, while there was no significant difference between ancestral nonthreatening stimuli $(M=.18)$ and ancestral threatening stimuli $(M=.17)$, $t(85)=.38($ see Figure 16$)$.

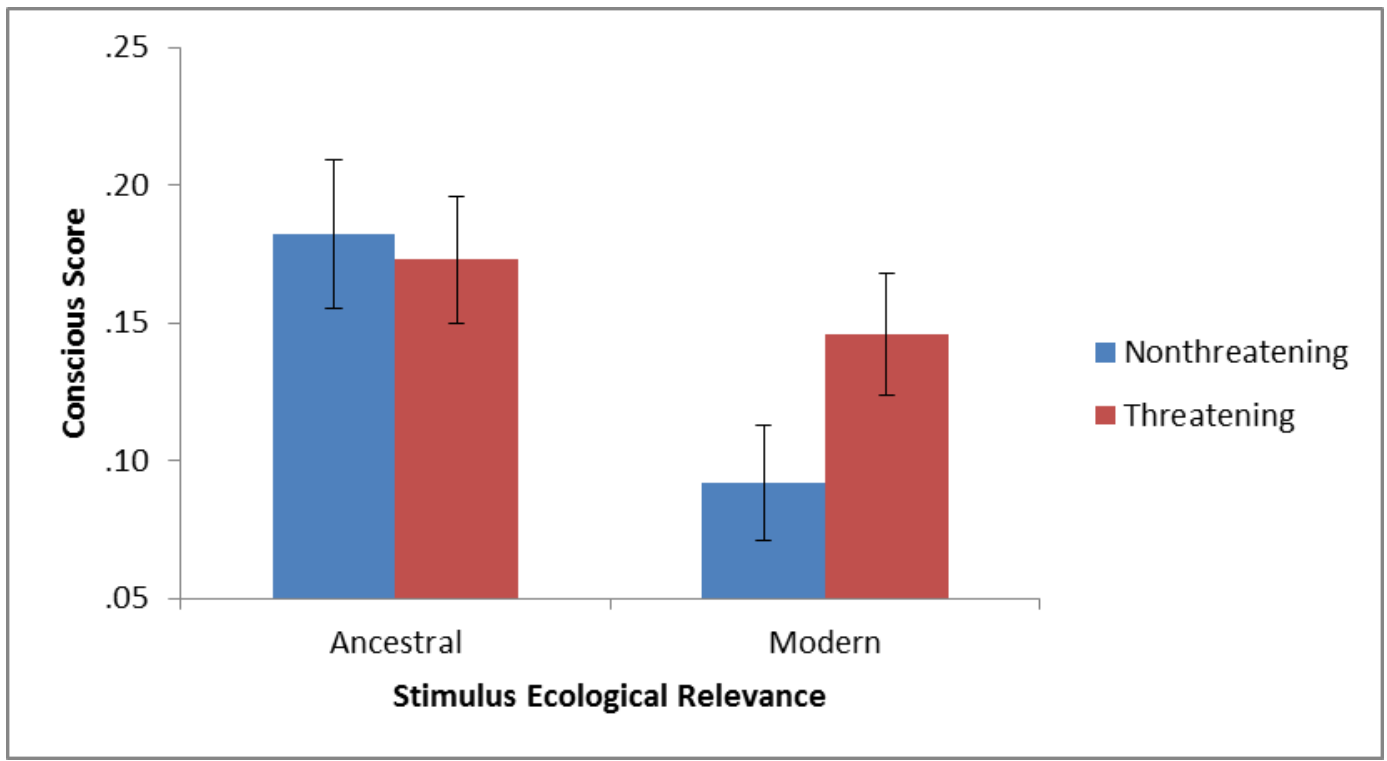

Figure 16. Conscious scores as a function of stimulus ecological relevance and threat level. Conscious scores represent the influence of conscious memory on performance on inclusion and exclusion trials. 
The only significant effect for unconscious scores was an interaction between threat level and stimulus ecological relevance, $F(1,83)=4.94, p=.029, \eta^{2}=.06$, whereby the influence of unconscious memory was greater for modern threatening $(M=.15)$ than modern nonthreatening $(M=.11)$ stimuli, $t(85)=2.44, p=.017$, while there was no difference between ancestral nonthreatening stimuli $(M=.13)$ and ancestral threatening stimuli $(M=.12), t(85)=.82($ see Figure 17$)$.

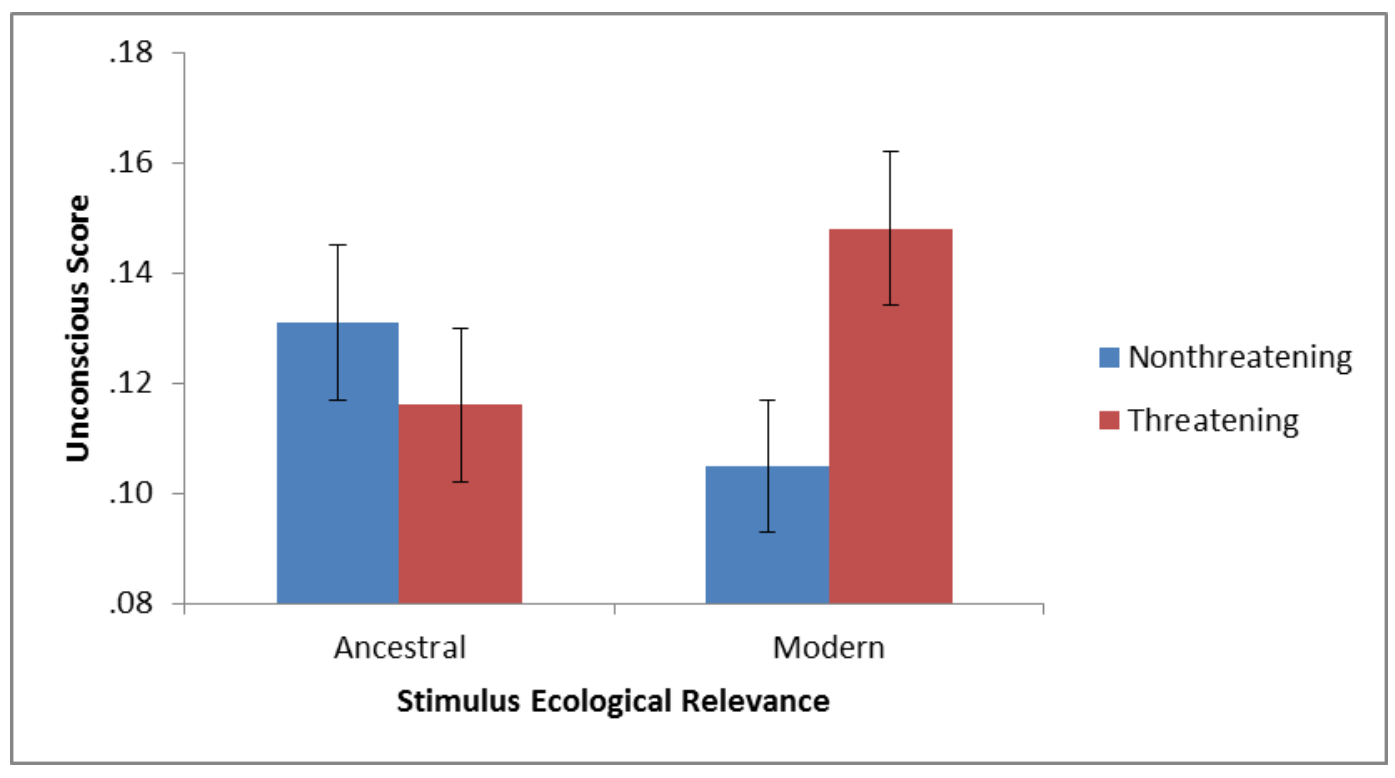

Figure 17. Unconscious scores as a function of stimulus ecological relevance and threat level. Unconscious scores represent the influence of unconscious memory on performance on inclusion and exclusion trials.

Rating Phase. Subjects rated how arousing, threatening, positive/negative, and survival relevant the individual stimuli were. Each rating was submitted to a separate 2 (threat level: nonthreatening or threatening) x2 (stimulus ecological relevance: ancestral or modern) within-subjects ANOVA. 
Arousal ratings. There was a significant main effect of threat level, $F(1,85)=$ $179.07, p<.001, \eta^{2}=.68$, whereby nonthreatening stimuli $(M=1.93)$ were rated as less arousing than threatening stimuli $(M=4.63)$. There was also a significant main effect of stimulus ecological relevance, $F(1,85)=88.52, p<.001, \eta^{2}=.51$, whereby ancestral stimuli $(M=3.70)$ were rated as more arousing than modern stimuli $(M=2.87)$. In addition to the main effects, there was also a significant interaction between threat level and stimulus ecological relevance, $F(1,85)=12.99, p=.001, \eta^{2}=.13$, whereby the difference in arousal rating between modern nonthreatening and threatening stimuli was greater than the difference between ancestral nonthreatening and threatening stimuli (see Figure 18).

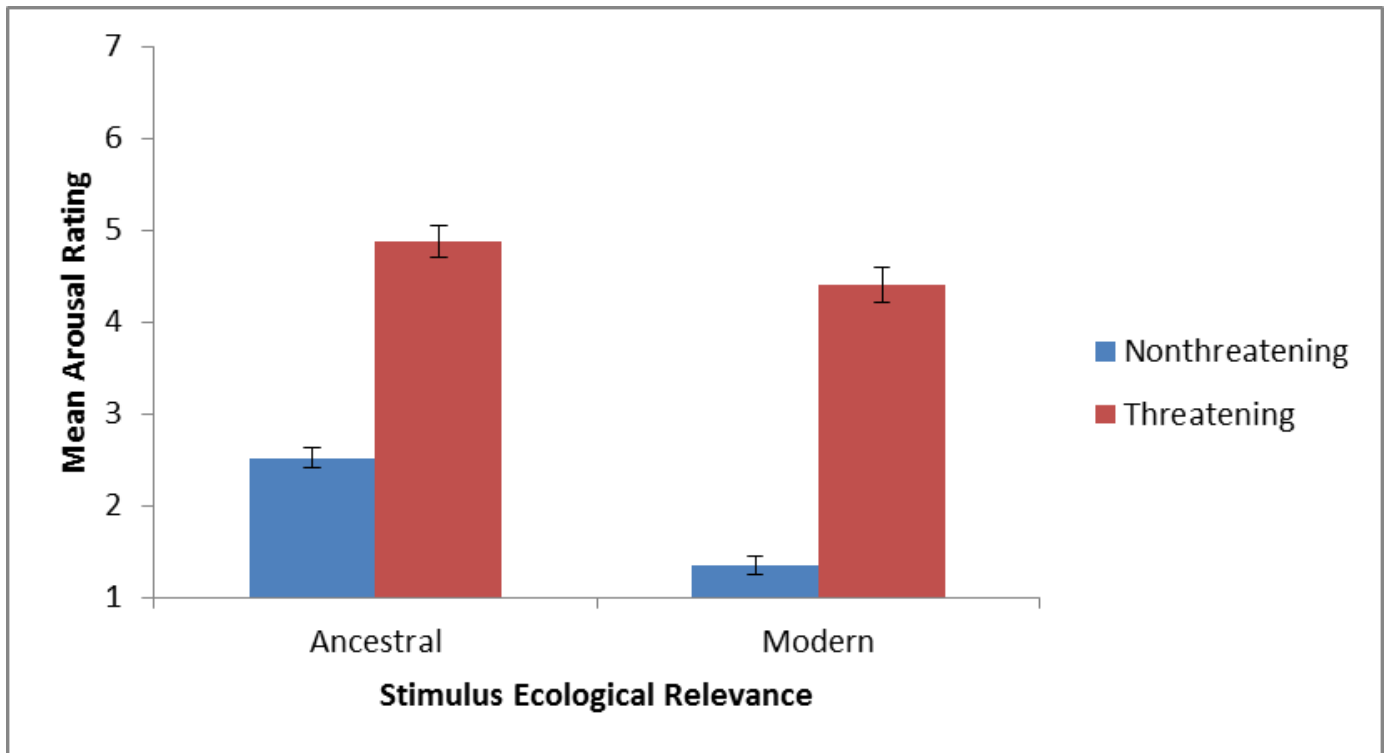

Figure 18. Arousal ratings as a function of stimulus ecological relevance and threat level.

Pairwise comparisons revealed that ancestral threatening stimuli $(M=4.87)$ were rated as significantly more arousing than ancestral nonthreatening stimuli $(M=2.52), t(85)=$ 
$10.86, p<.001$. Modern threatening stimuli $(M=4.40)$ were rated as significantly more arousing than modern nonthreatening stimuli $(M=1.35), t(85)=13.18, p<.001$. Also, ancestral nonthreatening and ancestral threatening stimuli were rated as significantly more arousing than modern nonthreatening stimuli, smallest $t(85)=9.45, p<.001$.

Threat ratings. There were main effects of both threat level, $F(1,85)=1,377.31$, $p<.001, \eta^{2}=.94$, and stimulus ecological relevance, $F(1,85)=43.12, p<.001, \eta^{2}=.34$. Nonthreatening stimuli $(M=1.43)$ were rated as less threatening than threatening stimuli $(M=5.43)$. Ancestral stimuli $(M=3.66)$ were rated as more threatening than modern stimuli $(M=3.21)$. The interaction between threat level and stimulus ecological relevance was not significant.

Positivity/negativity ratings. For positivity/negativity ratings there was a main effect of threat level, $F(1,33)=78.53, p<.001, \eta^{2}=.70$, whereby nonthreatening stimuli were rated as more positive $(M=4.42)$ than threatening stimuli $(M=2.60)$. The main effect was qualified by an interaction with stimulus ecological relevance, $F(1,33)=$ $152.64, p<.001, \eta^{2}=.82$, whereby ancestral nonthreatening stimuli $(M=4.73)$ were rated as more positive than ancestral threatening stimuli $(M=2.27), t(33)=11.17, p<.001$, while modern threatening stimuli $(M=2.92)$ were rated as more negative than modern nonthreatening stimuli $(M=4.10), t(33)=4.22, p<.001$ (see Figure 19$)$. 


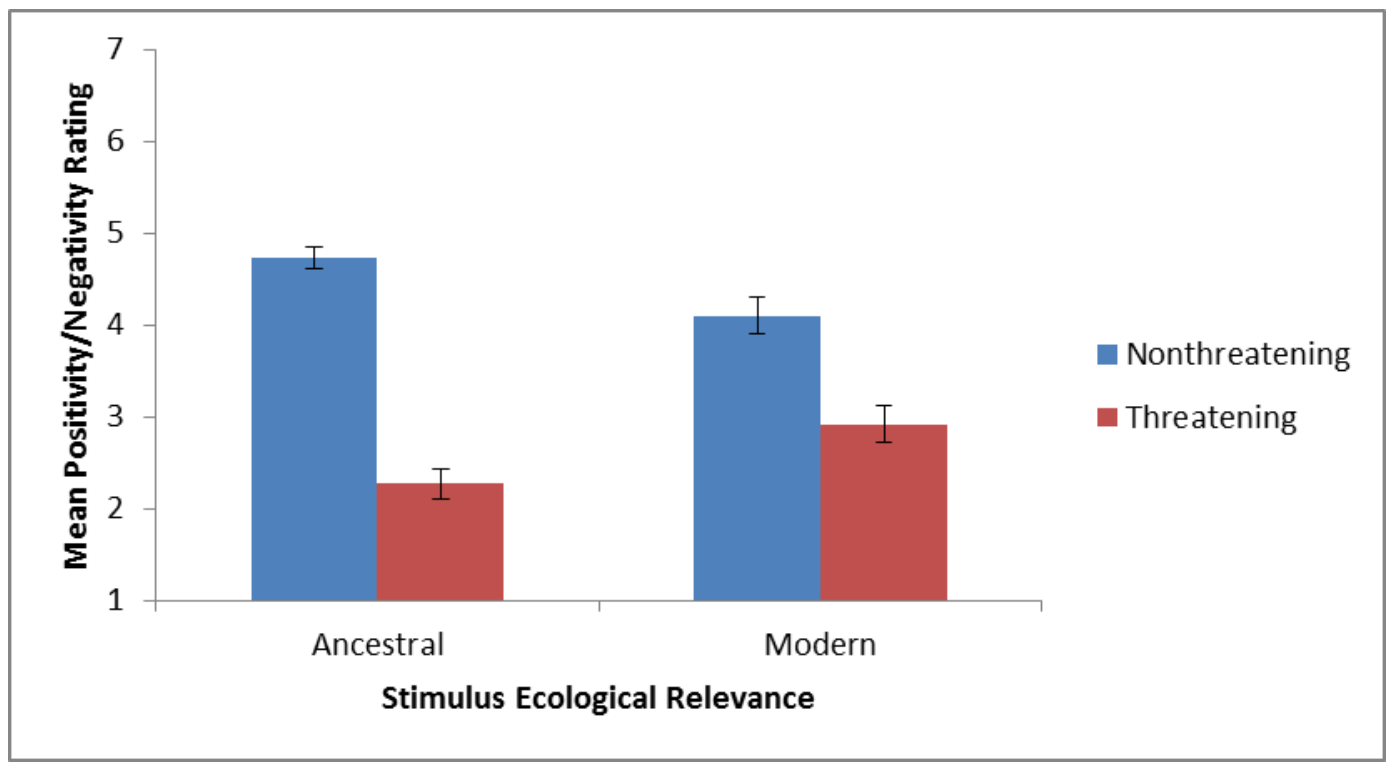

Figure 19. Positivity/negativity ratings as a function of stimulus ecological relevance and threat level.

Mean positivity/negativity ratings for all stimulus types were submitted to a onesample $t$ test against the scale's neutral point (4). Ancestral nonthreatening, ancestral threatening, and modern threatening stimuli all differed significantly from neutral (largest $p<.001$ ), but modern nonthreatening stimuli did not, $t(33)=0.53$.

Survival ratings. The only significant finding was a main effect of threat level, $F(1,33)=152.64, p<.001, \eta^{2}=.82$, whereby threatening stimuli $(M=5.17)$ were rated as more survival relevant than nonthreatening stimuli $(M=1.89)$.

\section{Further analyses.}

Item analyses. $\mathrm{RT}$ s to snakes $(M=943.61)$ and spiders $(M=977.98)$ were slower than RTs to other ancestral threatening stimuli $(M=935.55)$, which does not align with the predictions of the specific stimuli hypothesis. Response times to lions $(M=825.67)$ 
did align with predictions of the specific stimuli hypothesis, as they were faster than response times to other ancestral threatening stimuli, $t(10)=2.29, p=.045$.

Analyses of snake and spider stimuli did not align with predictions of the specific stimuli hypothesis for location memory, as assessed by the proportion of correct locations chosen during the inclusion task. In the inclusion task, the proportion of correct locations chosen for snakes $(M=.15)$ and spiders $(M=.27)$ were lower than the proportion of correct locations chosen for other ancestral threatening stimuli $(M=.29)$. Although the proportion of correct locations for lions $(M=.35)$ were higher than the proportion of correct locations chosen for other ancestral threatening stimuli, the difference was not significant, $t(4)=.84$. This does not provide strong support of the specific stimuli hypothesis.

\section{Discussion}

\section{Learning phase.}

Threatening versus nonthreatening stimuli. Subjects in the incidental condition performed the search task exactly as did subjects in Experiment 1. In this condition there was the typical main effect of threat level. However, there were no significant effects in the intentional or survival conditions. This pattern suggests that encoding style can affect whether threatening stimuli are responded to faster than nonthreatening stimuli. In the intentional and survival conditions, subjects were presumably trying to remember the locations where stimuli appeared because they knew their location memory would be tested afterwards and this may have caused subjects to pay more attention to each stimulus and not simply respond as soon as one was noticed. Subjects still may have 
detected threatening stimuli more rapidly than nonthreatening stimuli, but observed them slightly longer in an attempt to remember their location before responding. This is supported by research showing that threatening stimuli capture and hold attention more than nonthreatening stimuli (Koster et al., 2004). This is also supported by the fact that response times were numerically, albeit nonsignificantly, slower following intentional and survival instructions than incidental instructions.

Trees versus buildings. Tree-only grids were responded to significantly faster than building-only grids on target-absent trials, but there was no main effect of background ecological relevance on target-present trials: Targets were not located faster on tree grids than building grids. The faster response to tree-only grids than buildingonly grids may reflect the fact that trees are more arousing than, less common than, and preferred over buildings.

Other findings. There was a significant interaction between threat level and background ecological relevance in the incidental condition, whereby threatening stimuli were responded to faster than nonthreatening stimuli on ancestral backgrounds. The difference in response times to threatening and nonthreatening stimuli was not significant on modern backgrounds. This result was not observed elsewhere and is therefore not discussed further here.

\section{Memory phase.}

Inclusion trials. The inclusion task was essentially the same as the recall task in Experiment 1: Subjects had to choose the location where they saw the target stimulus. There was a significant interaction between threat level and stimulus ecological relevance 
which was driven by the fact that performance was lower for modern nonthreatening stimuli than for other stimulus types. The results of the inclusion task are best explained by general mnemonic principles, because modern nonthreatening stimuli were as the least arousing and most common stimuli. Modern nonthreatening stimuli were also nonvalenced (rated as neutral on positivity/negativity ratings).

Exclusion trials. The exclusion task was the reverse of the inclusion task: Subjects had to choose any location other than where the stimuli were seen. While both conscious and unconscious memory may lead subjects to choose a stimulus's actual former location on inclusion trials, only unconscious memory leads to the same response on exclusion trials.

The only significant result on exclusion trials was the interaction between threat level and stimulus ecological relevance. Among ancestral stimuli, the locations of nonthreatening stimuli were erroneously chosen more frequently than the locations of threatening stimuli, while the opposite was found for modern stimuli (threatening > nonthreatening). This will be discussed under the conscious and unconscious memory section below.

Conscious memory. There was a main effect whereby the influence of conscious memory was greater for ancestral than modern stimuli, but this was qualified by a marginally significant interaction with threat level. The interaction was such that the influence of conscious location memory was lesser for modern nonthreatening stimuli than all other stimulus types. This pattern is best explained by the GMP hypothesis. Modern nonthreatening stimuli were the least arousing and most common stimuli, and 
were considered neutral in terms of positivity/negativity. These results make sense because conscious memory is known lead to superior performance for arousing (Mather \& Sunderland, 2011), uncommon (Winograd, 1981), and valenced (Hamann et al., 1999) stimuli.

Unconscious memory. For unconscious memory, there was a significant interaction between threat level and stimulus ecological relevance. The influence of unconscious memory was significantly greater for modern threatening than modern nonthreatening stimuli. The influence of unconscious memory was numerically greater for ancestral nonthreatening than ancestral threatening stimuli, but this difference was not significant.

Conscious and unconscious memory. Better location memory for ancestral nonthreatening and modern threatening stimuli than modern nonthreatening stimuli seems to be driven by both conscious and unconscious memory, while superior location memory for ancestral threatening stimuli seems to be driven exclusively by conscious memory. An account in terms of general mnemonic principles can explain the greater conscious and unconscious influences of memory for ancestral nonthreatening and modern threatening stimuli than modern nonthreatening stimuli, but it is not obvious why the same pattern was not found for ancestral threatening stimuli. It may be that, as firstorder threats, subjects attended to ancestral threatening stimuli more than the other stimulus types during the learning phase, thus leading to a greater influence of conscious memory for these stimuli. 
Summary. The results of the search task indicated that performance of the task can be influenced by encoding instructions, as the typical threat advantage was found in the incidental encoding condition, but not the intentional or survival encoding conditions. The results of the inclusion task supported the GMP hypothesis, as location memory was worse for modern nonthreatening stimuli than all other stimulus types. The analyses of the memory tasks also provided evidence that better location memory for ancestral threatening stimuli than modern threatening stimuli may be driven by conscious memory, while better location memory for ancestral nonthreatening and modern threatening stimuli may be driven by both conscious and unconscious memory. 


\section{EXPERIMENT 3: IMPLICIT MEMORY}

\section{Introduction}

Experiments 1 and 2 involved explicit tests of memory; that is, subjects knew they were being tested on their location memory for previously seen stimuli. An argument can be made that explicit tests do not accurately reflect how memory is expressed in a natural setting. Consider once again the foraging scenario presented in the introductions to Experiments 1 and 2. Our hypothetical human had been wandering through the environment foraging for food and encountered a threatening stimulus, namely a snake, but managed to escape unharmed. This individual then goes wandering on the same path at a later date. We have previously assumed that the individual explicitly remembers the location where the threatening stimulus was encountered, either by recalling it before going near it or by recognizing it once in the presence of retrieval cues. However, it could be that the individual does not explicitly remember the location, but nonetheless remembers it implicitly. Implicit memory occurs when behavior is influenced by experience or learning without deliberate recall of the memory (Schacter, 1987). An example would be a doctor diagnosing a patient with the same diagnoses that the doctor had seen on a television show the night before, even though the doctor does not deliberately remember watching the show while making the diagnosis. In relation to the current scenario, implicit memory could manifest itself by causing the individual to 
respond faster when presented with the same threatening stimulus again in the same location, even without explicitly attempting to be more cautious.

Studies have often yielded evidence of implicit memory in the absence of explicit memory. Behavioral experiments have shown that amnesics show deficits on explicit tests of memory, but show less deficits or perform at normal levels on implicit tests (Graf \& Schacter, 1985; Schacter, Harbluk, \& McLachlan, 1984). In addition, both electrophysiological (Ko, Duda, Hussey, \& Ally, 2013; Rugg, Mark, Walla, Schloerscheidt, Birch, \& Allan, 1998) and neuroimaging (Schott, Henson, RichardsonKlavehn, Becker, Thoma, Heinze, \& Düzel, 2005) studies have shown dissociations in the brain areas activated during explicit and implicit memory trials (Voss \& Paller, 2008). If humans have been selected to have superior implicit but not explicit location memory for threatening stimuli, then an implicit test would obviously be needed to reveal support for the SLMTS hypothesis.

Experiment 3 tested implicit memory. A priming task was used to accomplish this, as explained below. The SLMTS hypothesis predicts that, when stimuli are presented in the same location in the memory phase as in the learning phase, the decrease in response times in the former will be larger for threatening stimuli than nonthreatening ones. The reason for this predicted decrease is that subjects will have stronger memories for the locations of threatening than nonthreatening stimuli, allowing for more rapid responses to threatening stimuli based on more rapid confirmation of expectations. The SLMTS hypothesis also predicts that, when stimuli are presented in a different location in the memory phase than the learning phase, there will be an increase in response times in the former that is larger for threatening stimuli than nonthreatening stimuli. The reason 
for this predicted increase is that subjects will have a strong memory for the location of the threatening stimulus in a different location, and it will take them longer to overcome this violation of expectations. Since they are not predicted to remember the locations of nonthreatening stimuli as well, their expectation of those stimuli in a different location will not be as great. This expectation may be conscious or unconscious, but it is not possible to distinguish between the two in this paradigm (Jacoby, 1991).

\section{Method}

Subjects. Sixty-three subjects (47 females, $M_{\text {age }}=19.71$ years, $S D=2.28$ ) participated. Subjects were recruited and selected according to the procedure listed in the General Method section above. A number of subjects were excluded from analyses for the following reasons: over 30 years old $(n=2)$, computer problems $(n=2)$, and response time or memory accuracy scores more than 2.5 standard deviations from the mean $(n=$ 3).

Materials and Procedure. All subjects first completed the learning phase. The learning phase was identical to that described in the General Method section. Following the learning phase there was a two-minute break during which subjects were asked to wait for further instructions.

After the two-minute break all subjects completed the memory phase. Subjects completed a task identical to that performed during the learning phase. Subjects again completed 64 target-present and 64 target-absent trials, using the same targets as in the learning phase. As in the learning phase, subjects' task was to press a key if all pictures in the grid were from the same category (e.g., nine trees) and to press a different key if 
one of the pictures came from a different category (e.g., eight trees and one snake). All target stimuli in the memory phase were presented on the same background as in the learning phase. However, in the memory phase, one exemplar from each stimulus type was presented in the same location as in the learning phase while the other exemplar was presented in a different location.

Subjects were randomly assigned to one of two conditions. In one condition, all stimuli that were presented on tree backgrounds in the learning phase were presented in the same location during the memory phase. In the other condition, all stimuli that were presented on building backgrounds in the learning phase were presented in the same location during the memory phase.

Difference scores were calculated between response times in the learning and memory phases (learning phase minus memory phase) for each stimulus category (e.g., ancestral nonthreatening stimuli in the same location). This allowed for within-subjects comparisons based on three factors: target threat level (nonthreatening versus threatening), target ecological relevance (ancestral versus modern), and memory phase location (same as/different than learning phase location).

Following the memory phase subjects completed the ratings phase. Subjects rated how threatening, arousing, and positive/negative stimuli were. Due to data logging issues, positivity/negativity ratings were not collected for five subjects (see General Method). 


\section{Results}

Learning phase. The dependent variable was RT. Incorrect responses were eliminated from RT data (less than $10 \%$ of the responses for each subject). Independent $t$ tests revealed no differences based on condition (stimuli presented on tree-grids or building-grids in same location during memory phase), $t(61)=.72$. All further analyses collapsed across this factor. On target-absent trials, a paired $t$ test revealed a significant difference in RT between background types, $t(62)=4.71, p<.001$, whereby tree-only grids $(M=876.17)$ were responded to faster than building-only grids $(M=1000.73)$ (see Figure 20).

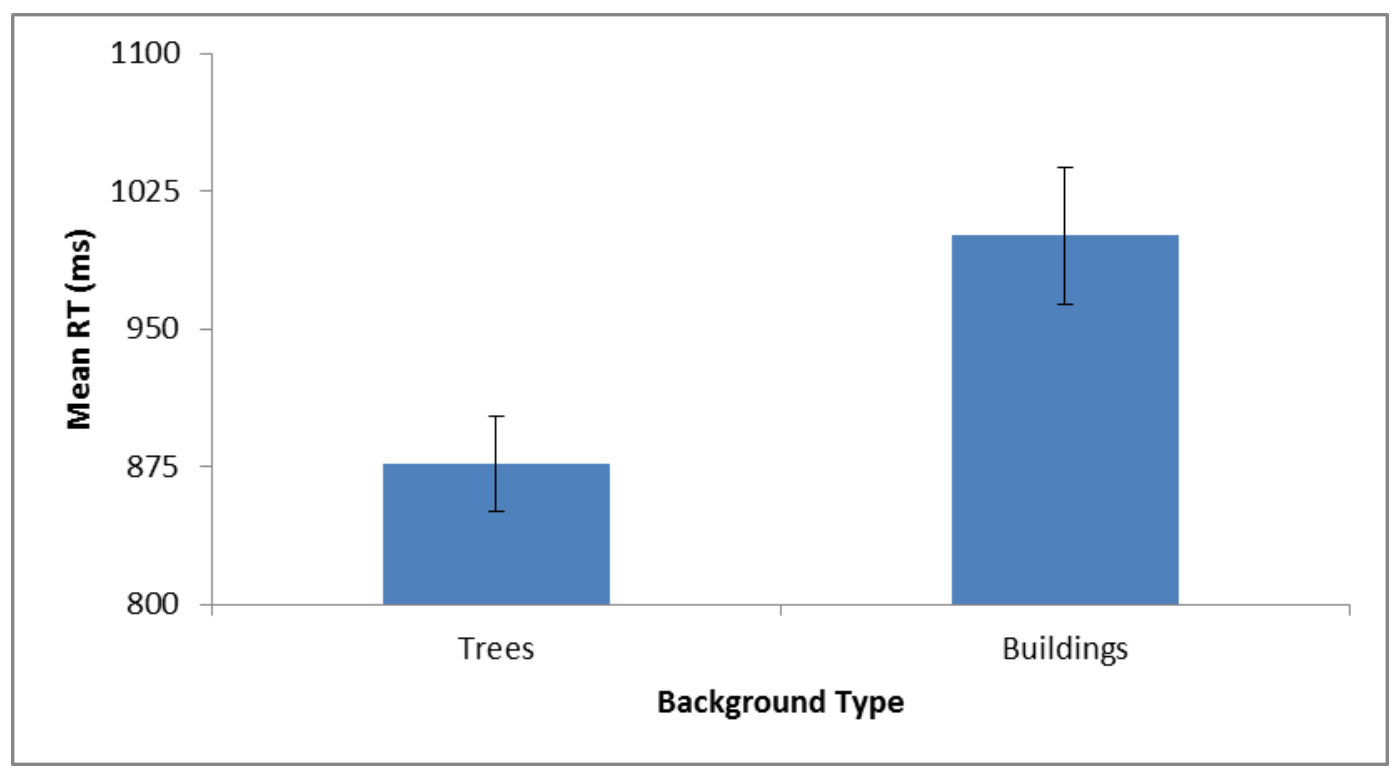

Figure 20. Mean RT on target-absent trials as a function of background type in the learning phase.

Following these initial analyses RTs on target-present trials were submitted to a 2 (threat level: nonthreatening or threatening) x2 (stimulus ecological relevance: ancestral or modern) x 2 (background ecological relevance: ancestral or modern) completely 
within-subjects. The only significant finding was a main effect of background ecological relevance, $F(1,62)=10.64, p=.002, \eta^{2}=.15$, whereby responses were faster to stimuli presented on ancestral backgrounds $(M=838.88)$ than on modern backgrounds $(M=$ 887.21). Unlike in Experiments 1 and 2, there was no main effect of threat level: RTs to threatening stimuli $(M=874.07)$ were not significantly different than RTs to nonthreatening stimuli $(M=852.01), F(1,62)=1.92$.

Memory phase. The dependent variable in the memory phase was RT. Incorrect responses were eliminated from RT data (less than $10 \%$ of the responses for each subject). Independent $t$ tests revealed no differences in RT based on condition, $t(61)=$ .09. All further analyses were collapsed across this factor. On target-absent trials, a paired $t$ test revealed a significant difference in RT between background types, $t(62)=$ 4.82, $p<.001$, whereby tree-only grids $(M=699.55)$ were responded to faster than building-only grids $(M=763.40)$ (see Figure 21$)$.

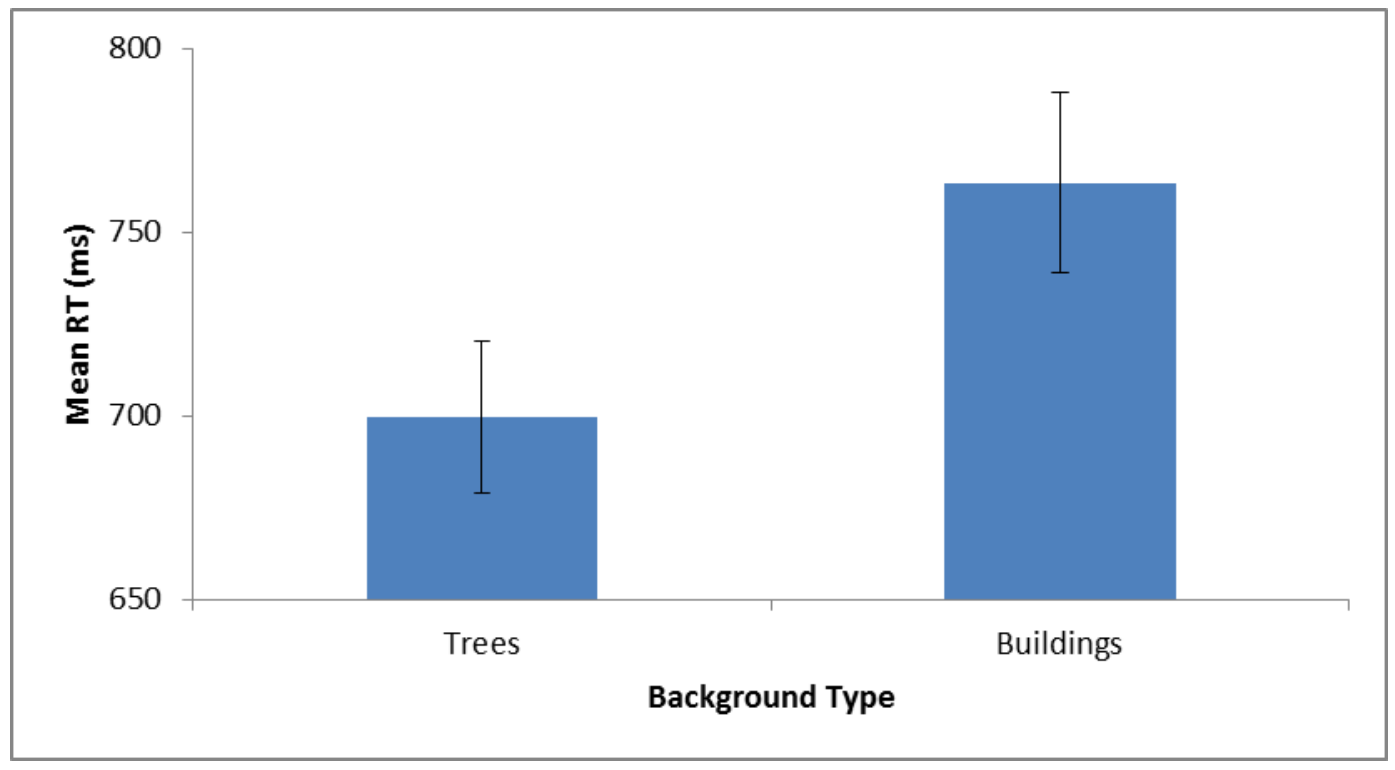


Figure 21. Mean RT on target-absent trials as a function of background type in the memory phase.

Following these initial analyses RTs on target-present trials were submitted to a 2 (threat level: nonthreatening or threatening) x2 (stimulus ecological relevance: ancestral or modern) x 2 (background ecological relevance: ancestral or modern) completely within-subjects ANOVA. There was a significant main effect of background ecological relevance, $F(1,62)=4.66, p=.035, \eta^{2}=.07$, whereby responses were faster to stimuli presented on ancestral backgrounds $(M=678.06)$ than on modern backgrounds $(M=$ 701.17). While responses were numerically faster to threatening stimuli $(M=680.51)$ than nonthreatening stimuli $(M=698.72)$, this difference only approached significance, $F(1,62)=3.53, p=.065, \eta^{2}=.05$.

Difference between learning and memory phases. The dependent variable was the difference score described in the Method section. Difference scores were submitted to a 2 (threat level: nonthreatening or threatening) x 2 (stimulus ecological relevance: ancestral or modern) x 2 (stimulus location: same or different) completely within-subjects ANOVA. There was a significant main effect of threat level, $F(1,62)=4.58, p=.036$, $\eta^{2}=.07$, whereby difference scores were greater for threatening $(M=193.56)$ than nonthreatening $(M=153.29)$ stimuli (see Figure 22$)$. 


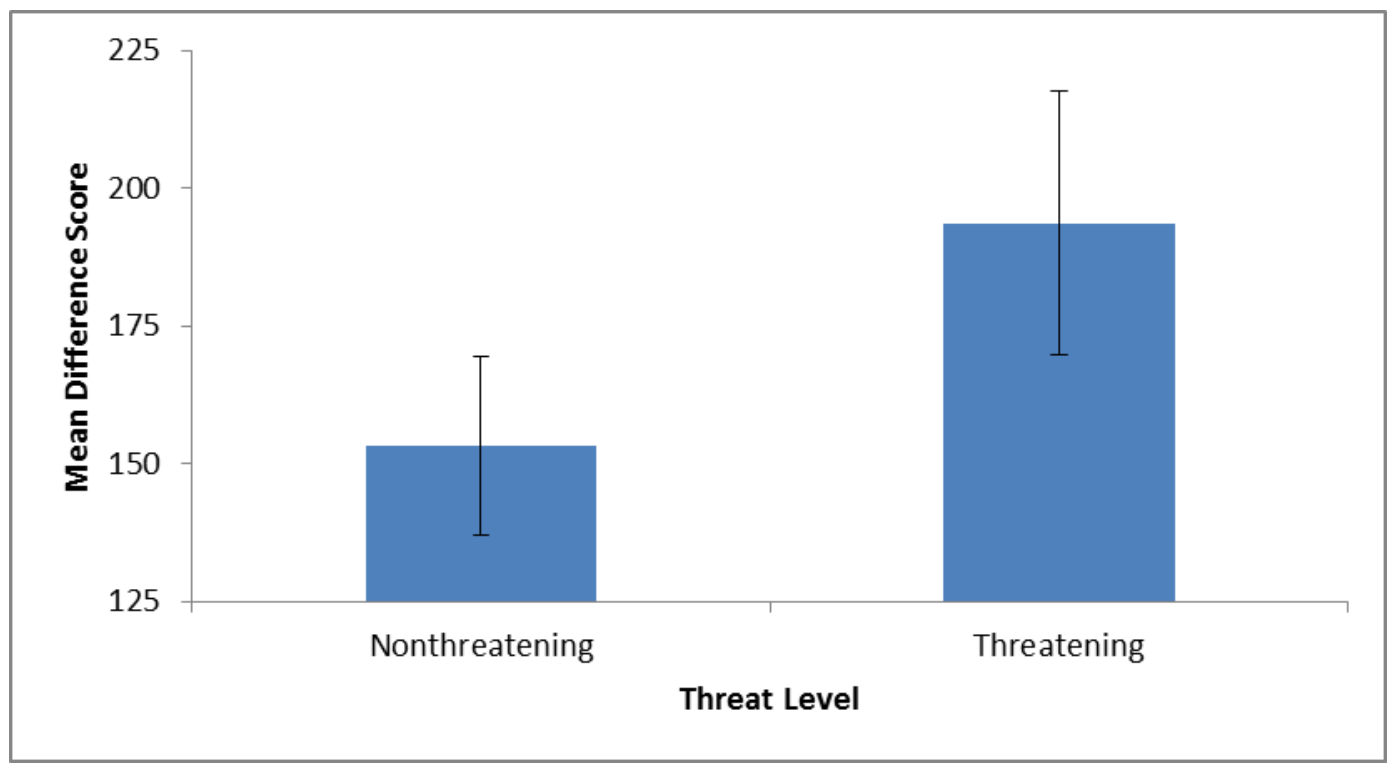

Figure 22. Mean difference score as a function of threat level.

The main effect was qualified by a significant interaction with stimulus location, $F(1,62)$ $=9.31, p=.003, \eta^{2}=.13$, whereby difference scores were greater for threatening $(M=$ 242.34) than nonthreatening stimuli $(M=133.78)$ when presented in the same location during the learning and memory phases, $t(62)=3.44, p=.001$, while difference scores did not significantly differ between nonthreatening $(M=172.80)$ and threatening $(M=$ 144.79) stimuli when presented in a different location during the memory phase, $t(62)=$ 1.05, (see Figure 23). 


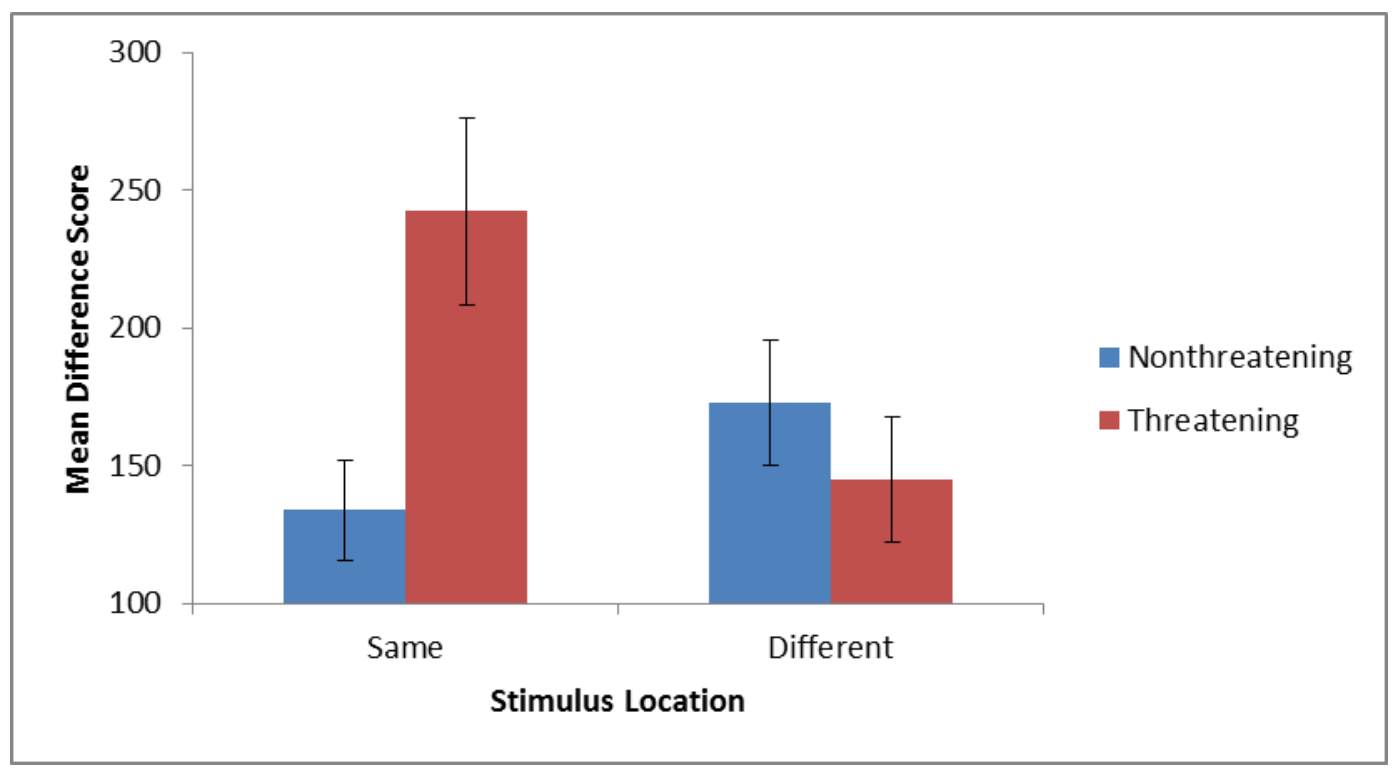

Figure 23. Mean difference scores as a function of stimulus ecological relevance and threat level.

Rating phase. Subjects rated how arousing, threatening, and positive/negative the individual stimuli were. Ratings were submitted to separate 2 (threat level: nonthreatening or threatening) x2 (stimulus ecological relevance: ancestral or modern) completely within-subjects ANOVAs.

Arousal ratings. There was a significant main effect of threat level, $F(1,62)=$ 105.31, $p<.001, \eta^{2}=.63$, whereby threatening stimuli $(M=4.33)$ were rated as more arousing than nonthreatening stimuli $(M=2.23)$. There was also a significant main effect of stimulus ecological relevance, $F(1,62)=108.79, p<.001, \eta^{2}=.64$, whereby ancestral stimuli $(M=3.83)$ were rated as more arousing than modern stimuli $(M=2.73)$. In addition to the main effects, there was also a significant interaction between threat level and stimulus ecological relevance, $F(1,62)=31.23, p<.001, \eta^{2}=.34$, whereby the 
difference in arousal ratings was greater between modern nonthreatening and threatening stimuli than between ancestral nonthreatening and threatening stimuli (see Figure 24).

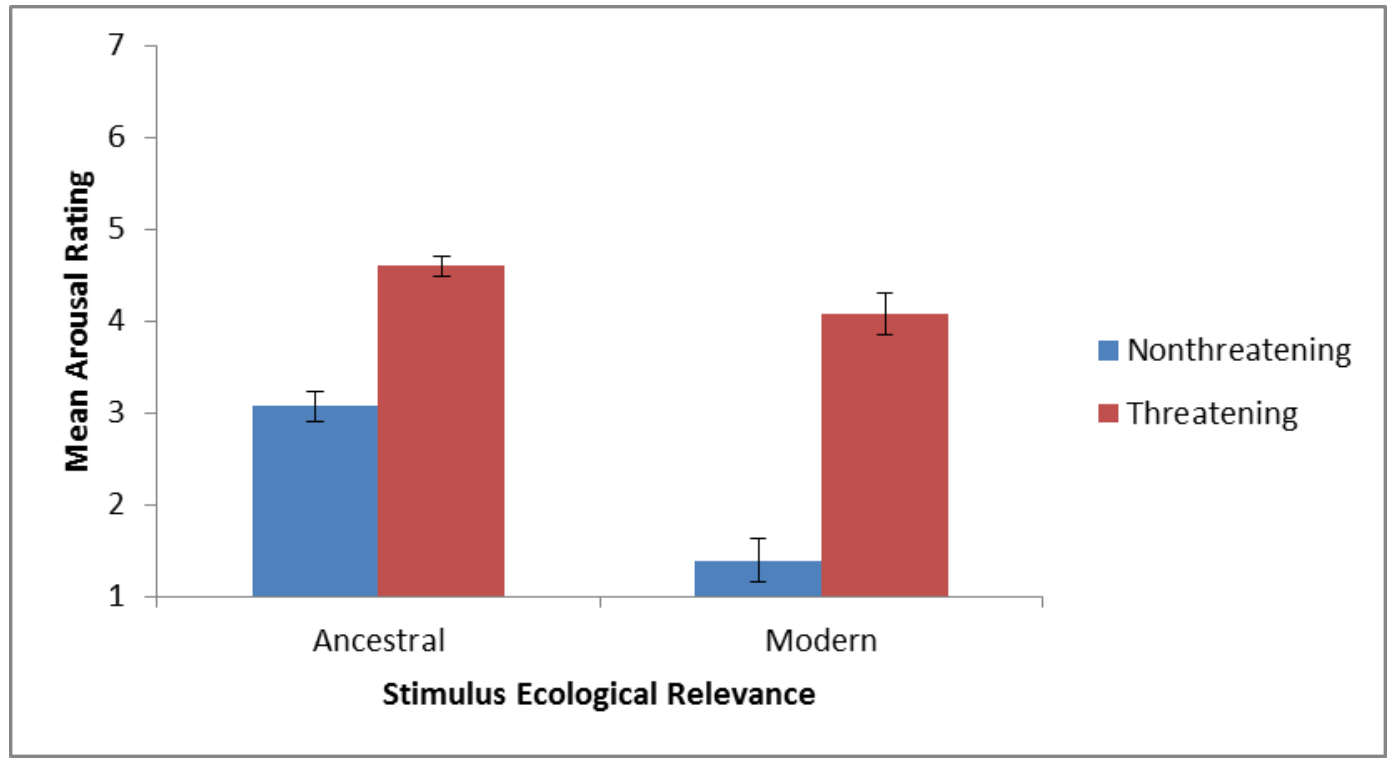

Figure 24. Arousal ratings as a function of stimulus ecological relevance and threat level.

Pairwise comparisons revealed that ancestral threatening stimuli $(M=4.60)$ were rated as significantly more arousing than ancestral nonthreatening stimuli $(M=3.07), t(62)=$ 7.07, $p<.001$. Modern threatening stimuli $(M=4.07)$ were also rated as significantly more arousing than modern nonthreatening stimuli $(M=1.39), t(62)=11.06, p<.001$. Also, ancestral nonthreatening and ancestral threatening stimuli were rated as significantly more arousing than modern nonthreatening stimuli, smallest $t(62)=9.67, p$ $<.001$.

Threat ratings. For threat ratings, there were main effects of both threat level, $F(1,62)=899.37, p<.001, \eta^{2}=.94$, and stimulus ecological relevance, $F(1,62)=27.42$, $p<.001, \eta^{2}=.31$. Threatening stimuli $(M=5.48)$ were rated as more threatening than 
nonthreatening stimuli $(M=1.37)$. Ancestral stimuli $(M=3.63)$ were rated as more threatening than modern stimuli $(M=3.21)$. The interaction between threat level and stimulus ecological relevance was not significant.

Positivity/negativity ratings. There was a main effect of threat level, $F(1,57)=$ $168.74, p<.001, \eta^{2}=.75$, whereby nonthreatening stimuli were rated as more positive $(M$ $=4.50)$ than threatening stimuli $(M=2.61)$. There was also a significant main effect of stimulus ecological relevance, $F(1,57)=13.40, p=.001, \eta^{2}=.19$, whereby modern stimuli $(M=3.33)$ were rated as more negative than ancestral stimuli $(M=3.78)$. There was also a significant interaction between threat level and stimulus ecological relevance, $F(1,57)=9.46, p=.003, \eta^{2}=.14$, whereby the difference between positivity/negative scores was greater between nonthreatening and threatening ancestral stimuli than nonthreatening and threatening modern stimuli (see Figure 25).

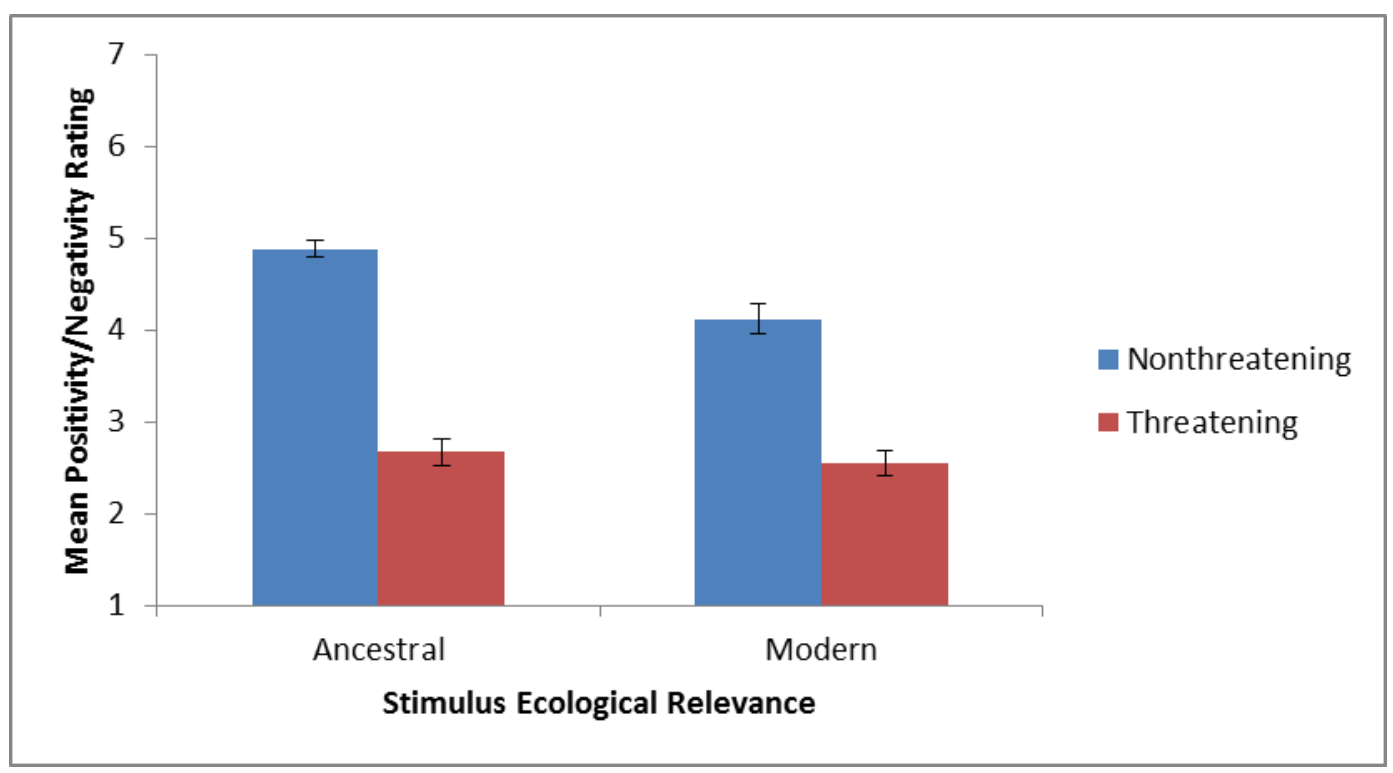

Figure 25. Positivity/negativity ratings as a function of stimulus ecological relevance and threat level. 
Pairwise comparisons revealed that ancestral nonthreatening stimuli $(M=4.88)$ were rated as significantly more positive than ancestral threatening stimuli $(M=2.67), t(57)=$ $14.15, p<.001$. Modern threatening stimuli $(M=2.55)$ were rated as significantly more negative than modern nonthreatening stimuli $(M=4.12), t(57)=7.91, p<.001$.

Mean positivity/negativity ratings for all stimulus types were submitted to a onesample $t$ test against the scale's neutral point (4). Ancestral nonthreatening, ancestral threatening, and modern threatening stimuli all differed significantly from neutral (largest $p<.001$ ), but modern nonthreatening stimuli did not, $t(57)=0.70$.

\section{Further analyses.}

Item analyses. Although $\mathrm{RTs}$ to snakes $(M=876.81)$, spiders $(M=875.98)$, and lions $(M=814.24)$ were numerically faster than RTs to other ancestral threatening stimuli $(M=893.87)$, these differences were not significant, largest $t(10)=0.97$. This does not provide strong support for the specific stimuli hypothesis.

Analyses of difference scores, like response time, for snake, spider, and lion stimuli did not produce results consistent with the predictions of the specific stimuli hypothesis. Difference scores were lower for spiders $(M=185.72)$ and lions $(M=80.94)$ than other ancestral threatening stimuli $(M=222.96)$. Although difference scores were numerically larger for snakes $(M=227.52)$ than other ancestral threatening stimuli, this difference was not significant, $t(10)=0.05$. This does not provide strong support for the specific stimuli hypothesis. 


\section{Discussion}

\section{Learning phase.}

Threatening versus nonthreatening stimuli. Response times did not differ based on threat level of the stimuli. This is in opposition to the results of Experiment 1 and Experiment 2 following incidental instructions. Since the search tasks were the same in all three experiments and similar to those used in past research that found a threat advantage, one possibility is that the failure to find a significant effect in this experiment was a Type II error.

Trees versus buildings. On target-absent trials, subjects responded significantly faster to tree-only grids than building-only grids. This may be due to the fact that trees are more arousing and less frequently encountered than, and preferred to, buildings. This rapid response to tree-only grids was reflected on target-present trials: Targets presented on tree grids were responded to faster than targets presented on building grids. Although potentially interesting, this effect has little bearing on how other results from the search task are interpreted, because none of the central findings in this dissertation involved a main effect of or interaction with background ecological relevance.

Differences between learning and memory phases. There was a significant main effect of threat level, which was qualified by an interaction with stimulus location. As predicted by the SLMTS hypothesis, when stimuli were presented in the same location in the memory phase as in the learning phase, the decrease in response times was larger for threatening than nonthreatening stimuli. When stimuli were presented in a different location in the memory phase than the learning phase, it is less clear that the 
prediction of the SLMTS hypothesis was supported. Response times in this condition decreased, rather than increasing, as was expected. This is likely because overall performance increased greatly from the learning phase to the memory phase, with responses becoming much faster. Nonetheless, even though response times to threatening stimuli decreased, the decrease was numerically smaller than for nonthreatening stimuli, which could be seen as at least somewhat consistent with the SLMTS hypothesis.

Summary. The results of Experiment 3 supported the SLMTS hypothesis. One could argue that the results do not actually reflect memory, but rather two separate search tasks. This argument is credible, but is undercut by the interaction between threat level and stimulus location when analyzing the difference scores. If the search task in the memory phase was truly separate and did not reflect memory, stimulus location should not have affected the results. Since location did affect the results, it is clear that memory is being assessed.

The results, and the above argument, then suggest that the SLMTS hypothesis is supported for implicit memory. It is important to note again that implicit memory does not necessarily mean unconscious memory. Implicit memory tasks can, theoretically, be completed using both conscious and unconscious memory processes, even though many authors argue that implicit tasks measure unconscious memory specifically (Jacoby, 1991). Potential differences between the results of the three experiments will be discussed in the General Discussion. 


\section{GENERAL DISCUSSION}

Research has consistently shown that humans respond more rapidly to the presence of threatening than nonthreatening stimuli (Blanchette, 2006; Brosch \& Sharma, 2005; Fox et al., 2007; LoBue, 2010a; Öhman, Flykt, \& Esteves, 2001). More recent research has also indicated that the locations of threatening stimuli may be remembered better than the locations of nonthreatening stimuli (Kuhbander et al., 2011; Wilson et al., 2011). The current research was motivated by a desire to combine these areas of research and determine whether the threat superiority effect in response times translates into a threat superiority effect in location memory. A combination of these areas is important for theorizing about how the cognitive system is designed to process different types of stimuli. The three experiments were therefore designed to replicate the threat superiority effect in search tasks while simultaneously testing the SLMTS hypothesis.

\section{Hypotheses}

In addition to the SLMTS hypothesis, several other hypotheses relevant to the current dissertation were described in the Introduction. These hypotheses, and how the results of the three experiments relate to them, will be discussed in detail below.

Null hypothesis. The null hypothesis states that there should be no difference in location memory as a function of stimulus threat level or ecological relevance. The results of each of the three experiments contradicted the predictions of this hypothesis. Therefore, the null hypothesis can safely be rejected. 
Specific stimuli hypothesis. This hypothesis states that humans have been adapted to deal with certain stimuli that were recurrent threats to humans in the ancestral environment, such as snakes (Isbell, 2006; Öhman \& Mineka, 2001), spiders (LoBue, 2010b; Rakison \& Derringer, 2008), and lions (Penkunas \& Coss, 2013). Modern humans should therefore respond to these stimuli more rapidly than other stimuli and have better location memory for them. Of the three stimuli in question, only lions were consistently responded to numerically faster than other ancestral threatening stimuli. Location memory was not consistently better for any of these stimuli than for other ancestral threatening stimuli. These results do not support the predictions of the specific stimuli hypothesis, and therefore it is reasonable to reject this hypothesis.

Specific features hypothesis. This hypothesis states that certain stimuli that were recurrent threats to humans in the ancestral environment should be responded to faster, and their locations should be remembered better than other stimuli, based on certain features of those stimuli. The only evidence supporting this hypothesis relates to the coiled shape of snakes (LoBue \& DeLoache, 2011; Masataka et al., 2011). Therefore, even though the shape of snakes was not manipulated in the current experiments, the specific stimuli hypothesis predicts that snakes should be responded to faster than other stimuli and their locations should be remembered better than those of other stimuli. However, as mentioned above, response times to snakes were not consistently faster than response times to other ancestral threatening stimuli and location memory was not consistently better for snakes than other ancestral threatening stimuli. This hypothesis, therefore, does not account for the results found in this dissertation and is therefore rejected. 
Ancestral threatening stimuli hypothesis. This hypothesis states that all ancestral threats should be responded to faster, and their locations should be remembered better than, those of other stimuli. Although threatening stimuli were responded to more rapidly than nonthreatening stimuli, there was no interaction with stimulus ecological relevance, and both ancestral and modern threatening stimuli were responded to equally rapidly. There was also no indication that location memory was better for ancestral than modern threatening stimuli.

There was, however, one interesting finding in the recognition condition of Experiment 1 relating to this hypothesis. The false alarm rate was especially high for ancestral threatening stimuli. This shows that there may be something unique about these stimuli. Because ancestral threatening stimuli are first-order threats, this elevated false alarm rate was explained as subjects possibly using a better-safe-than-sorry heuristic in the recognition condition. Therefore, although ancestral threatening stimuli may be deemed unique in relation to other stimuli, this uniqueness may come from individuals learning that these stimuli are first-order threats. This would contradict the ancestral threatening stimuli hypothesis, which argues that these stimuli are unique because humans have been specifically adapted to process them. Therefore, it is reasonable to reject the ancestral threatening stimuli hypothesis.

Ancestral stimuli hypothesis. This hypothesis states that humans have been adapted to deal with all ancestral stimuli. Ancestral nonthreatening stimuli were relevant to survival because they could be preyed upon and they could also alert humans as to the locations of other resources or oncoming dangers. Ancestral threatening stimuli were relevant to survival because they were generally predators or other dangerous animals 
that needed to be avoided. The ancestral stimuli hypothesis therefore predicts that the locations of ancestral stimuli should be responded to faster and remembered better than the locations of modern stimuli. Across the three experiments there was no evidence that ancestral stimuli were responded to faster than modern stimuli. In Experiment 2 the locations of ancestral stimuli were remembered better in the inclusion task than the locations of modern stimuli, but this main effect was qualified by an interaction with the threat level of the stimuli. There was no other indication that the locations of ancestral stimuli were remembered better than the locations of modern stimuli. In light of these results, it is reasonable to reject the ancestral stimuli hypothesis.

Survival processing hypothesis. This hypothesis states that humans have been adapted to process stimuli in terms of their relevance to personal survival. Based on this, locations of stimuli processed in terms of their survival relevance should be remembered better than the locations of stimuli processed in some other way (Nairne et al., 2012). While Nairne (2010) has argued that the survival processing enhancement in memory is independent of the stimuli used, there is some indication that that may not be the case (Butler, Kang, \& Roediger, 2009), and that certain stimuli, namely survival relevant stimuli, themselves may elicit survival processing (Howe \& Derbish, 2010).

Only Experiment 2 provided a test of this hypothesis. Location memory was not better in the survival encoding condition of that experiment than in either the incidental or intentional encoding conditions. Analyses on survival ratings in that experiment showed that threatening stimuli were rated as significantly more survival relevant than nonthreatening stimuli, and therefore it could be that threatening stimuli themselves elicit survival processing. However, there were no main effects of threat level in the memory 
results of Experiment 2, and the locations of threatening and nonthreatening stimuli were remembered equally well. In light of these results it is reasonable to reject the survival processing hypothesis in the current dissertation.

SLMTS hypothesis. This hypothesis states that location memory should be better for threatening stimuli than nonthreatening stimuli. This hypothesis is based on a wealth of research showing that threatening stimuli are responded to more rapidly than nonthreatening stimuli (Blanchette, 2006; Brosch \& Sharma, 2005; Fox et al., 2007; LoBue, 2010a; Öhman, Flykt, \& Esteves, 2001). Testing the SLMTS hypothesis, while simultaneously replicating the threat superiority effect in a search task, was the main motivation of the current dissertation.

For the most part, the threat superiority effect for response times was replicated in the current experiments. Threatening stimuli were responded to more rapidly than nonthreatening stimuli in Experiment 1 and in Experiment 2 when typical incidental instructions were used. It should be noted that this effect seems malleable, and that changing the instructions of the search task altered the results. This is elaborated on under the Future Directions heading below.

Support for the SLMTS hypothesis was mixed. Experiments 1 and 2 used explicit memory tests of conscious and unconscious location memory. The results of these experiments failed to support the SLMTS hypothesis: Location memory was not better for threatening than nonthreatening stimuli. Therefore, in terms of explicit memory, it seems reasonable to reject this hypothesis. In contrast, Experiment 3 used an implicit test of location memory and priming was greater for threatening than nonthreatening stimuli 
when stimuli were presented in the same location during the memory phase as the learning phase. This conforms to the predictions of the SLMTS hypothesis. This leads to two important questions. First, is there a reason why the results should differ for explicit and implicit tests of location memory? Second, how much does this one finding support the SLMTS hypothesis?

The question of whether the results from explicit and implicit memory tests should be different can actually be broken down into three parts. One, do results ever vary between explicit and implicit memory tests? Two, do varying results reflect different underlying memory systems? Three, is there any reason to believe that these systems should lead to different results in the current experiments?

The answer to the first part of the question is the clearest as it can be definitively said that results do indeed vary between explicit and implicit tests in certain cases (Roediger, 1990). There is even evidence that results on implicit tests are sometimes the direct opposite of results on explicit tests. One example is that pictures are remembered better than words during free recall (an explicit memory test), while words receive more priming than pictures on implicit tests of memory (Weldon \& Roediger, 1987).

Even though it is clear that results vary between explicit and implicit tests of memory, there has been a long debate as to whether this is because the two types of memory are supported by separate systems. Researchers who argue against the multiple systems view point out that most tests of implicit memory do not actually dissociate between conscious and unconscious memory systems, as they claim to do (Jacoby, 1991). They also make the point that differences between the two tests may reflect different 
processes, rather than different systems, similar to how results vary between recall and recognition tests even though they are both forms of explicit memory tests that are supported by the same memory system (Roediger, 1990). On the other side of the argument are researchers who believe that explicit and implicit memory tests are supported by separate underlying explicit and implicit memory systems. Early evidence in support of this view came from studying amnesics. Amnesic patients show deficits relative to control subjects in the retention of learned material when queried by explicit tests of memory. This difference, however, decreases or disappears when amnesics and control subjects are queried using implicit tests of memory (Schacter, 1987). Further support came from findings in normal subjects that explicit and implicit tests often produced different results (Schacter, 1992).

In addition to behavioral research, measurements of neural activity have been used to examine whether differences between explicit and implicit memory are caused by different processes of the same system or by separate systems. Voss and Paller (2008) performed a review of fMRI and ERP studies that investigated the neural underpinnings of both explicit and implicit memory in the same investigation. They concluded that there are differences between the underlying neural processes in explicit and implicit memory, and that these differences reflect multiple systems of memory.

Hence, there is clear evidence that separate systems of explicit and implicit memory exist. However, it is not assumed that these differences necessarily reflect differences between conscious and unconscious memory, as implicit memory tasks may theoretically be completed using conscious memory, as discussed in the discussion section of Experiment 3. This brings us to the third part of the question. 
There are two reasons why the results of the current experiments may differ for explicit and implicit memory. One reason is that, while explicit tests of memory in the current dissertation have not supported the SLMTS hypothesis, past research using an implicit test of memory did support this hypothesis (Li et al., 2008), at least for anxious individuals. The second reason is that the priming test used in Experiment 3 represents a rapid response based on memory. It is likely that a rapid response is more important to threatening than nonthreatening stimuli, because they can pose an immediate danger to survival. Therefore, the immediate danger posed by threatening stimuli may be the most relevant factor for the implicit memory system, even if it is not for the explicit memory system.

As has been explained, there is reason to believe that the results of the experiments in this dissertation should vary depending on whether explicit or implicit memory was tested. This allows for an argument to be made that there is tentative support for the SLMTS hypothesis, at least for implicit memory. Future research will need to replicate and extend the results of Experiment 3 to provide greater support this hypothesis; this point will be discussed further in the Future Directions section below.

GMP hypothesis. The GMP hypothesis states that location memory should be better for stimuli that are more arousing (Mather \& Sunderland, 2011), less common,(Barclay, 2008; Winograd, 1981), and more strongly valenced (positively or negatively) (Hamann et al., 1999) than other stimuli. As discussed above, ancestral nonthreatening stimuli, ancestral threatening stimuli, and modern threatening stimuli were all rated as significantly more arousing than modern nonthreatening stimuli. Ancestral nonthreatening stimuli were always rated as positive while ancestral and 
modern threatening stimuli were always rated as negative. Modern nonthreatening stimuli were mostly rated as neutral, and if these stimuli are valenced, they are only weakly so compared to the other stimulus types. Finally, modern nonthreatening stimuli are among the most common stimuli encountered on a daily basis, while the stimuli belonging to all other categories are encountered less frequently. The GMP hypothesis therefore predicts that location memory should be better for all stimulus types other that modern nonthreatening stimuli in the current experiments.

The results of Experiments 1 and 2 conformed to the predictions of the GMP hypothesis, because the locations of all other stimulus types were, generally, remembered better than the locations of modern nonthreatening stimuli. This is possibly even true of the recognition task in Experiment 1, if one takes the better-safe-than-sorry heuristic into consideration. In that task, the accuracy was better for ancestral nonthreatening and modern threatening stimuli than modern nonthreatening stimuli, as predicted by the GMP hypothesis. Overall accuracy did not, however, differ between ancestral threatening and modern nonthreatening stimuli due to the high false-alarm rate to ancestral threatening stimuli. As first-order threats, a high false-alarm rate to these stimuli may be beneficial. In this way the results of the recognition task supported the GMP hypothesis. The results of Experiment 3 did not, however, conform to the predictions of this hypothesis. As discussed above, Experiments 1 and 2 tested explicit memory, while Experiment 3 tested implicit memory. There is reason to believe that the results of the current experiments should vary depending on whether explicit or implicit memory is tested. Therefore, we can say that the results of Experiments 1 and 2 provide strong support for the GMP hypothesis specifically in explicit memory. 


\section{Natural selection as unifying factor}

As shown above, two separate hypotheses have been supported by the current experiments. This is not unreasonable, given that there may be separate explicit and implicit systems of memory. Nevertheless, the theory of natural selection presents a common underlying principle that somewhat unifies the two hypotheses, as will be explained below. Before expanding on this common principle, it is first necessary to explain the way in which natural selection does not unify these two hypotheses.

Domain-specific natural selection. When people think of natural selection, they tend to think of domain-specific adaptations caused by natural selection. This is very common in the field of evolutionary psychology, where many researchers believe that the brain contains modules that have been selected for over many generations to solve specific and recurrent problems posed by the natural environment (Chiappe \& MacDonald, 2005). This emphasis on domain-specificity is not surprising because there are many examples of domain-specific adaptations in nature ${ }^{17}$ such as those responsible for bacterial resistance to anti-bacterial drugs. Also, domain-specific explanations of behavior often possess face validity and offer direct cause-effect relationships that are easily understood.

However, not all adaptations are domain-specific, and domain-specific adaptations do not always provide the best explanation of phenomena. In relation to the current dissertation, it is difficult to see how domain-specific selection would be responsible for the disparate results for explicit and implicit memory. There is no one particular threat that humans needed to remember the location of that would account for 
both of these results. This, however, does not mean that natural selection cannot unify the hypotheses that were supported by the results of the current experiments.

Domain-general natural selection. In addition to domain-specific adaptations, plasticity, or the ability to respond in a variety of ways given the personal experiences and current circumstances of an organism (West-Eberhard, 1989), can also be naturally selected for. Adaptations that are plastic in nature are also referred to as domain-general adaptations. These adaptations are more difficult to illustrate, because they are generally not individual physical features but rather patterns of behavior. An example would be plasticity being selected for in terms of detection of and memory for dangerous stimuli. Recent studies show that individuals with a history of enduring abuse are better than safely nurtured individuals on detection and memory tasks involving dangerous stimuli (Frankenhuis \& de Weerth, 2013). Frankenhuis and de Weerth argue that this reflects the fact that our cognitive systems are plastic, and develop to best process information that is most relevant to each individual.

It is here argued that location memory follows a domain-general principle, and that humans may have been selected to follow this principle. This domain-general principle is that the individual should remember the information that is most relevant dependent upon the situation. For explicit memory, this means remembering the locations of stimuli that are more arousing, less common, and more valenced than other stimuli, as stated in the GMP hypothesis. For implicit memory, this means remembering the locations of stimuli that can pose an immediate threat and require a rapid behavioral response. In this way, the domain-general principle of relevancy to current goals unifies 
the SLMTS and GMP hypotheses, and explains why they were each supported in different contexts.

\section{Differences between attention and memory}

Across the three experiments, there was a consistent RT advantage for threatening stimuli over nonthreatening stimuli, but this did not translate into a consistent location memory advantage. Given the overlap between attention and memory described in the Introduction, the existence of an RT effect in the absence of a memory effect requires an explanation. One possible explanation relies on the relevance of the stimuli in particular situations.

When searching the environment, threatening stimuli are of particular relevance, because they can pose an immediate risk to the survival of the organism. On the other hand, when remembering the locations of stimuli in the environment there is no immediate threat and threatening stimuli are not necessarily more relevant than other stimuli (with the possible exception of implicit memory, as has been explained). For example, it is also relevant to remember the locations of watering holes, sources of food, sources of firewood and other useful natural resources, the locations of potential mates, and the locations of potential trading partners. Although other explanations may be possible, this explanation shows how the principle of relevancy can account for the differences between attention and location memory findings in the current experiments.

\section{Relation to past research}

Memory for the locations of threatening stimuli. As discussed in the Introduction, there is a large literature on response time to threatening stimuli, but the 
literature on location memory for threatening stimuli is comparatively minimal. To date, only two studies have specifically tested memory for the locations of threatening stimuli. Both of those studies tested explicit memory. The results of each of those studies will be revisited, and how they relate to the GMP hypothesis will be discussed.

Iconic memory for threatening stimuli. Kuhbander et al. (2011) compared neutral, positive, and threatening stimuli for iconic memory decay rates and transfer from iconic to short-term memory. When considering the ratings of the stimuli used in their study, the results seem to match the predictions of the GMP hypothesis. Threatening and positive stimuli were rated as valenced and their locations were transferred from iconic to short-term memory at higher rates than the locations of neutral stimuli, which were rated as non-valenced. In addition, the locations of threatening stimuli decayed from iconic memory at a slower rate than both neutral and positive stimuli, which were both rated as less arousing than threatening stimuli. The iconic memory test used in Kuhbander et al.'s experiment tested explicit memory. Therefore, the results agree with the results of the two explicit memory tests of the current dissertation (Experiments 1 and 2).

All of these results fit with the underlying principle that individuals should remember information that is most relevant in the current situation. This principle also accounts for differences between decay rate in iconic memory and transfer from iconic to short-term memory. While it may be relevant to transfer memories of both positive and negative stimuli from iconic to short-term memory, a slower decay rate may be particularly important for negative (threatening) stimuli. Threatening stimuli can pose a potential immediate risk and a slower decay rate may lead to decreased response times to these stimuli (Kuhbander et al., 2011). 
Longer-term memory for threatening stimuli. Wilson et al. (2011) tested location memory for threatening stimuli using a long-term explicit memory test. Specifically, the test was a game of pelmanism with threatening stimuli as targets and nonthreatening stimuli as fillers. Each subject played the game of pelmanism two times, once with ancestral threatening stimuli as targets and once with modern threatening stimuli as targets. While ancestral and modern threatening stimuli were matched on arousal ratings, ancestral threatening stimuli were rated as more negative.

Wilson et al. (2011) found that fewer errors were made towards ancestral than modern threatening stimuli. They also found that when errors were made the distance was greater for modern than ancestral threatening stimuli. The authors argued that their results support the ancestral threatening stimuli hypothesis, and that their results reflect the fact that humans have been selected to remember the locations of stimuli that were threatening in the ancestral environment. These results are also in line with the GMP hypothesis which states that location memory should be better for stimuli that are more arousing, uncommon, and valenced than other stimuli. In Wilson et al.'s study, the GMP hypothesis would predict that location memory for ancestral threatening stimuli should be better than for modern threatening stimuli, because the former were more arousing and valenced.

Explicit memory for the locations of emotional stimuli. The GMP hypothesis

does not only account for results from experiments that specifically tested threatening stimuli. Mather and Nesmith (2008) performed four experiments explicitly testing whether location memory was better for arousing than non-arousing stimuli. None of the stimuli they used represented a threat to survival ${ }^{18}$. The locations of arousing stimuli 
were remembered better than the locations of non-arousing stimuli across all four experiments. These results also conform to the GMP hypothesis, and therefore match those of the explicit memory tests in this dissertation. The results also agree with the domain-general principle of goal-relevancy and support the arguments made in this dissertation about the role of goal-relevancy in location memory.

\section{Future directions}

Superior implicit location memory for threatening stimuli. Although the results of Experiment 3 supported the SLMTS hypothesis, this was only one experiment. The GMP hypothesis was supported in two separate tests of explicit memory and using tests of both recall and recognition, therefore providing a large degree of support. In order to more strongly support the SLMTS hypothesis for implicit memory, the results of Experiment 3 must be replicated. This would preferably be accomplished by means of a different test of implicit location memory than the one used in Experiment 3. If memory was again found to be better for threatening than nonthreatening stimuli, this would be strong support for the SLMTS hypothesis in relation to implicit memory.

Goal relevancy. It has been suggested that the results of the current experiments can be explained by the domain-general principle that individuals best remember the locations of information that is most relevant to them in the current situation. While this hypothesis has been supported by the current results, further research is needed. It could be that explicit and implicit location memory systems truly do function differently, and that there is no unifying principle between the two. This is an empirical question, and suggestions for testing this principle are described below. 
The results of Experiment 3, which tested implicit memory, supported the SLMTS hypothesis. It was suggested that these results also supported the unifying domain-general principle that the locations of stimuli deemed relevant in the current situation are remembered the best, because threatening stimuli are particularly important to the implicit memory system because they potentially require rapid responses. Although support for the SLMTS hypothesis in Experiment 3 was in line with the domain-general principle of goal-relevancy, this may not always be the case.

In order to dissociate between the SLMTS hypothesis and the underlying domaingeneral principle of goal-relevancy in implicit location memory, a situation must be set up where nonthreatening stimuli are deemed more relevant to the implicit memory system than threatening stimuli. Such a situation would lead to differing predictions of the implicit memory results. The SLMTS hypothesis would predict that location memory for threatening stimuli should be better than for nonthreatening stimuli while the principle of goal-relevancy would predict the opposite.

There is reason to believe that the results of such a study would support the principle of goal-relevancy, rather than the SLMTS hypothesis. Gibson and Bahrey (2005) showed that strength of priming depends on congruency between learning and testing modalities, possibly supporting the importance of goal-relevancy in priming. Furthermore, Holbrook et al. (2003) had subjects study two types of words: those that were relevant to the completion of a task and those that were not relevant. The authors found priming for stimuli that were relevant to task demands, but no priming for stimuli that were not relevant. This showed that implicit memory is sensitive to task relevancy. 
The results of Experiments 1 and 2 supported the GMP hypothesis for explicit memory. It was argued that this hypothesis was supported in this case because it aligned with the underlying principle of goal relevancy. Although stimuli that are predicted to be remembered best by the GMP hypothesis (i.e., stimuli that are arousing, uncommon, and valenced) are generally also the most relevant to current goals, this is not always the case. An experiment could be designed in which location memory for stimuli which are less arousing, more common, and less valenced is more goal relevant than location memory for stimuli that are more arousing, less common, and more valenced. In such a case the GMP hypothesis would predict superior location memory for the latter group of stimuli but the underlying principle of goal-relevancy would predict better location memory for the former.

The domain-general principle of goal-relevancy has the explanatory power to account for the results of the experiments in this dissertation, as well as other experiments that have been discussed. This would provide a unifying link between the differing results found for explicit and implicit memory in the current study. Future research specifically testing this domain-general principle will allow for stronger conclusions to be drawn.

Influence of encoding style on search tasks. Experiment 2 varied encoding style before subjects performing the search task. Only in the typical, incidental encoding condition were threatening stimuli responded to significantly faster than nonthreatening stimuli. This possibly shows that encoding style affects performance on the search task. Future research is needed to determine whether this is in fact the case. 
Individual differences. The current experiments used a convenience sample of students at the University of Louisville. Although all subjects were college students, this does not mean they were otherwise homogenous. Subjects presumably varied along many dimensions of personal experience. Future research might consider these individual differences and how they might affect the results. Some examples of potentially relevant differences include: place of origin, amount of time spent in different environments (e.g., urban, suburban, rural), personal experiences with the stimuli, occupation, and experiences with pets.

\section{Conclusions}

This dissertation was motivated by research in the field of evolutionary psychology. It is common for evolutionary psychologists to favor domain-specific hypotheses, and to argue that the brain consists of discrete modules that were selected for to allow for rapid solutions to recurring problems in the environment (Cosmides \& Tooby, 2013). One such module is suggested to exist for location memory, because humans have been selected to remember the locations of threatening stimuli, and especially stimuli which posed recurrent threats to the survival of our ancestors (Wilson et al., 2011). The purpose of the current dissertation was to investigate this claim.

Taken together, the three experiments of this dissertation do not support the idea that human location memory is domain-specific or that it has adapted and evolved in response to recurrent threats. Instead, the results support the domain-general idea that individuals remember the locations of stimuli that are most important or relevant given current circumstances. While this goal relevancy led to enhanced memory for the 
locations of threatening stimuli in implicit memory, it led instead to enhanced memory for the locations of arousing, uncommon, and positive or negative stimuli in explicit memory, regardless of whether the stimuli were threatening or not.

It is important to note that this conclusion does not rule out the possibility of domain-specific nerve patterns in humans. Indeed innate reflexes, such as the rooting reflex, are examples of domain-specific adaptations. It may also be the case that humans possess cognitive pathways that have been selected to specifically process certain information that was recurrent and important to the survival or reproduction of our ancestors. Possible examples include responding to threatening stimuli (LoBue et al., 2010), detecting cheaters in a social exchange (Cosmides, 1989), sex differences in mating strategies (Buss, 1998), and sex differences in spatial location abilities (Cosmides \& Tooby, 2013).

While domain-specific adaptations do exist in humans, and are possibly important in our everyday cognitive functioning, this does not appear to be the case for location memory. Instead of the human location memory system having evolved in response to a recurrent problem of needing to remember the locations of specific threats, it is more likely that it evolved in response to the general problem of needing to remember the locations of stimuli that were relevant to current goals. The goals of individuals shift constantly. This is as true for ancestral humans as well as those living today. Our system of location memory is not rigid, but flexible, allowing us to meet our goals in an ever changing environment. 


\section{ENDNOTES}

1) The theory of natural selection, as proposed by Charles Darwin and refined over the past century and a half, has been defined by Ernst Mayr (2001, p. 288) as "the process by which in every generation individuals of lower fitness are removed from the population". Although this definition is not perfect by itself, as it at least requires a definition of the term fitness, it is a nice simple formulation that allows for further explanation of the theory. In rough terms the definition states that individuals, regardless of the type of life they are, have variable characteristics. To at least some degree these characteristics are heritable, meaning they can be passed on to their progeny. This variability in characteristics leads some individuals to be better suited to survive and reproduce in the environment they live in than other individuals. Those individuals who are best suited to survive and reproduce will be the most likely to actually reproduce and pass on those heritable characteristics to further generations. Those individuals who are less well suited will be less likely to pass on their characteristics that hinder them from being well suited to survive and reproduce. After successive generations only those characteristics that best suit individuals to survive and reproduce will still be extant in the population.

2) The two major forces that drive the evolution of species are natural selection and genetic drift, or random chance. The field of evolutionary psychology focuses 
exclusively on the force of natural selection for several reasons. The primary reason is that, of the two forces, most current evolutionary researchers believe that natural selection plays a greater role in evolution than genetic drift (Dawkins, 1976; but see Kimura, 1991). The second reason is that natural selection is the more interesting force to researchers in psychology because it allows for a "because" explanation; that is that researchers can use the theory of natural selection to claim that we have a certain mental or behavioral trait because it allowed our ancestors to solve a certain and specific adaptive problem in the past. When traits evolve due to genetic drift or random chance, there is no reason to propose a "because" explanation, because that trait did not evolve as a solution to a problem.

The exclusive use of natural selection in evolutionary psychology can lead to potential problems. If researchers are specifically looking for selected traits, they may find them where they do not exist; in other words evolutionary psychologists are at a risk of creating Type I errors. Some traits may appear to be useful, but may indeed have evolved due to genetic drift rather than because of a selective pressure (Gould \& Lewontin, 1979). It may also be that a trait evolved for one reason, but is currently used to serve some other purpose (Gould \& Vrba, 1982). These problems lie at the heart of many criticisms of evolutionary psychology specifically and adaptationist tendencies in psychology and biology more generally.

3) Care must always be taken when using the term 'trait' because of the many meanings that the word has in different scientific fields and in everyday use. For 
the sake of this dissertation a trait will be referred to as a discrete, heritable characteristic. Such a characteristic may be physical or mental. An example of a physical trait would be the size of the horns on a beetle. If one beetle has particularly large horns, and this trait helps the beetle win more mates, then he will pass that trait on to subsequent generations. Mental traits are somewhat more difficult to describe, because they cannot be easily pictured. An example of a mental trait would be a baby duck imprinting on the first living creature it sees, and following it around, whether or not it is the duck's mother.

4) It could be argued that many flowers and mushrooms posed as much of, or even more of, a threat to humans than many of the species of snakes that our ancestors would have encountered. Of course there are many varieties of flowers and mushrooms that are poisonous and can lead to serious sickness or death if contacted or ingested, while there are many varieties of snakes that are nonvenomous and too small to cause damage by choking or biting. Although this is true, it should still be noted that, in terms of immediate threats to survival, snakes posed a much greater risk of threat to survival than plants, if for no other reason than that flowers and mushrooms are immobile, while many snakes strike at extremely rapid speeds. While one could take time in deciding whether to ingest a flower or mushroom, the detection of and reaction to snakes needed to be immediate if humans were to avoid a potentially dangerous or deadly bite.

5) While the terms ancestral and modern are used in this paper, other terms can also be used to describe the stimuli, such as: evolutionarily relevant and evolutionarily irrelevant, natural and manmade, or animate and inanimate. The terms ancestral 
and modern could be criticized because one could argue that some stimuli referred to as modern were also used by ancestral humans (such as knives). Nevertheless, they are used here because they are simple, easy to remember, and descriptive, and they refer to timeframes that highlight the influence of natural selection; natural selection has had time to work on ancestral stimuli but not necessarily more modern stimuli.

6) Innate is another term that requires defining because of the various interpretations that are possible. For the purposes of this dissertation the word innate will refer to something that is phenotypically expressed because of the way that it is encoded in the genotype. Although the phenotypic characteristic may first be expressed at any point during development, it is independent of the effects of the environment surrounding the organism in question. In the current instance, an innate pathway means that ancestral threatening stimuli are detected rapidly because the organizational pattern in the brain that leads to this rapid detection is encoded in the genome and requires no special environmental conditions or individual experiences for it to be phenotypically expressed. The stimuli which elicit firing in this pathway are engrained in the genetic code of the individual and will elicit firing in the pathway even in the absence of experience.

7) The difference between an emotional circuit and a survival circuit requires some explanation, as the concepts are not widely known. An emotional circuit refers to a theoretical pattern of firing in the brain, in response to certain stimuli, which produces something we would consider as a recognizable emotion, such as fear, anger, happiness, or love. The problems with this concept include: 1) such 
circuits have yet to be found, 2) a wide variety of stimuli seem to elicit these discrete emotions, and that the stimuli may in fact elicit different emotions depending on the context or even the mood of the observer, 3) there is a longstanding debate as to which emotions should be considered basic and therefore which emotional circuits should be present in the brain, and 4) the emotional circuits do not necessarily lead to predictable patterns of behavior when elicited. On the other hand, there is good evidence for the existence of survival circuits in the brain (see LeDoux, 1998). An example of a survival circuit would be a pattern of brain firing in a mouse when the smell of a predator, such as a cat, is detected. This presence elicits a probabilistic avoidance, escape, or attack behavior, depending on the current surroundings of the mouse. What we call emotions may in fact be labels given to the arousal we feel when these survival circuits are elicited.

8) In the adaptive memory paradigm, subjects typically read one of two or more scenarios. One of the scenarios is always set up to cause subjects to feel as if they are living in an ancestral environment where they must survive; an example is a scenario explaining that subjects are stranded in a savannah grassland and must acquire food and avoid predators. Control scenarios ask subjects to imagine themselves in some other situation, one that does not imitate how our ancestors would have lived; an example is that subjects must imagine they are moving and must decide what is important. After reading their scenarios all subjects read a list of words. Subjects are then asked to rate the words for how relevant they are 
to the scenarios they had previously read. After rating the words subjects take a memory test for the words they just rated.

9) Humans are generally not very good at completing the Wason Selection Task, as first used by Wason. In this version subjects must solve a logic problem that is represented by only numbers and letters. Cosmides reframed the problem using humans and actions in the place of numbers and letters. She found that when the logic problem is framed in a way that forces humans to detect cheaters, performance is greatly improved.

10) If superior memory for the faces of cheaters existed, then all others who were not remembered could be assumed to be cooperators or fair individuals. If all other individuals, aside from those remembered to be cheaters, were seen as fair and cooperators, it would allow for cooperative exchange with those individuals to proceed regardless of if they are remembered or not, unless they were later found to be cheaters. There is evidence that humans generally consider others to be trustworthy during initial exchanges with them (or what would be thought of as an initial exchange if the previous encounter with the individual was forgotten). The tit for tat model of social exchange, where on an initial encounter all individuals are treated as cooperators, but afterwards they are treated based on their previous behavior, has been shown to be one of the most effective models of exchange (Axelrod \& Hamilton, 1981) and would only require memory for the faces of cheaters, as is proposed here. 
11) Even though the authors did not make any claims about a comparison between threatening and nonthreatening stimuli a look at their reported data seems to indicate that differences did indeed exist. It appears that ancestral (what they refer to as evolutionarily relevant) threatening stimuli produced fewer errors than ancestral nonthreatening stimuli, as would be expected based on their predictions and their discussion. However, it also seems that modern (what they refer to as evolutionarily irrelevant) threatening stimuli produced fewer errors than evolutionarily irrelevant nonthreatening stimuli. This point is not discussed by the authors and seems to undermine their point that only the locations of ancestral threatening stimuli should be remembered to a greater extent, because humans have been specially adapted to remember the locations of such stimuli.

12) Isbell's work uses models and comparisons that support her argument. As a model she notes the similarity between the visual systems of raptors that specialize in eating snakes with the visual systems of catarrhines (old world monkeys). She argues that this similarity is indicative of analogous evolution of the visual systems in the two groups due to a similar selective pressure; namely the need to detect snakes rapidly, even though in the case of the raptors this rapid detection is to capture prey while in the case of the catarrhines this rapid detection is to avoid predators. As additional evidence in support of her argument, Isbell compares the visual systems of catarrhines and platyrrhines. Catarrhines have a much longer and more consistent history of coexistence with venomous snakes than platyrrhines (or new world monkeys). As a result the visual systems and brain structures of old world monkey species are more likely to be adapted to 
snake detection. Among other characteristics, this includes a higher regularity of trichromacy among the catarrhines than the platyrrhines.

13) Of the two snake exemplars used in the current experiments, one is in an elongated position while the other is slightly coiled, though not in a typical striking position. Although these shapes do vary, the differences are not great enough and there are not enough exemplars used to allow for comparisons based on stimulus shape.

14) As an example of this, two of the stimuli that were labeled as ancestral threatening stimuli were bears and sharks. These two stimuli can be very dangerous to humans, and accordingly both were rated as very threatening by the subjects in the current experiments. Having said that, it is almost certainly the case that these stimuli have not had a large selective pressure on the evolution of humans or other primates. Bears, while certainly dangerous to modern and ancestral humans, were not predators of humans and were likely only dangerous when a human attacked a bear or its cubs or accidentally got in its way. The case is even clearer with sharks. Although sharks are extremely dangerous, have been known to prey on humans, and inspire scary movies, we have never lived in the same environment and avoiding sharks certainly does not require a specific adaptation, all one has to do is stay out of the water!

15) All subjects also completed a modified version of Oldfield's (1971) Edinburgh Handedness Inventory (see Lyle, McCabe, \& Roediger, 2008 for details). The Edinburgh Handedness Inventory collects information about age, sex, and hand 
preference for a variety of daily activities. Based on responses to the Edinburgh Handedness Inventory, subjects were divided into two handedness groups (consistent or nonconsistent). As none of the results differed based on consistency of handedness, this factor receives no mention in the main body of the paper.

16) This significant interaction for false alarms qualified a main effect of threat level, $F(1,40)=4.15, p=.048, \eta^{2}=.09$, whereby false alarms were more likely for threatening $(M=.36)$ than nonthreatening $(M=.32)$ stimuli.

17) For a list of examples see Bocco in Works cited.

18) One of the stimuli did picture an individual holding a gun to their own head. This was a negative and highly arousing stimulus. While the picture did contain the image of a gun, the stimulus is not considered threatening because the obvious danger is to the actor in the image, not to the observer. 


\section{REFERENCES}

Awh, E. \& Jonides, J. (2001). Overlapping mechanisms of attention and spatial working memory. Trends in Cognitive Sciences, 3, 119-126.

Axelrod, R. \& Hamilton, W. D. (1981). The evolution of cooperation. Science, 211, 1390-1396.

Balling, J. D. \& Falk, J. H. (1982). Development of visual preference for natural environments. Environment and Behavior, 14, 5-28.

Bannerman, R. L., Temminck, E. V., Sahraie, A. (2012). Emotional stimuli capture spatial attention but do not modulate spatial memory. Vision Research, 65, 12-20.

Barclay, P. (2008). Enhanced recognition of defectors depends on their rarity. Cognition, 107, 817-828.

Barclay P. \& Lalumière, M. L. (2006). Do people differentially remember cheaters? Human Nature, 17, 98-113.

Barrett, L. F. (2011) Was Darwin wrong about emotional expressions? Current Directions in Psychological Science, 20, 400-406.

Barrett, L. F., Mesquita, B., \& Gendron, M. (2011). Context in emotion perception. Current Directions in Psychological Science, 20, 286-290.

Barrett, L. F., Tugade, M. M., \& Engle, R. W. (2004). Individual differences in working memory capacity and dual-process theories of the mind. Psychological Bulletin, $130,553-573$. 
Beall, P. M. \& Herbert, A. M. (2008). The face wins: Stronger automatic processing of affect in facial expressions than words in a modified Stroop task. Cognition \& Emotion, 8, 1613-1642.

Becker, M. W. \& Detweiler-Bedell, B. (2009). Early detection and avoidance of threatening faces during passive viewing. Quarterly Journal of Experimental Psychology, 62, 1257-1264.

Bell, R. \& Buchner, A. (2010). Valence modulates source memory for faces. Memory \& Cognition, 2010, 29-41.

Bell, R. \& Buchner, A. (2012). How adaptive is memory for cheaters? Current Directions in Psychological Science, 21, 403-408.

Blanchette, I. (2006). Snakes, spiders, guns, and syringes: How specific are evolutionary constraints on the detection of threatening stimuli? Quarterly Journal of Experimental Psychology, 59, 1484-1504.

Bocco, D. 10 Examples of natural selection. Discovery. http://dsc.discovery.com/tvshows/curiosity/topics/10-examples-natural-selection.htm

Brosch, T. \& Sharm, D. (2005). The role of fear-relevant stimuli in visual search: A comparison of phylogenetic and ontogenetic stimuli. Emotion, 5, 360-364.

Buchner, A., Bell, R., Mehl, B., \& Musch, J. (2009). No enhanced recognition memory, but better source memory for faces of cheaters. Evolution and Human Behavior, $30,212-224$.

Burns, D. J., Hwang, A. J., \& Burns, S. A. (2011). Adaptive memory: Determining the proximate mechanisms responsible for the memorial advantages of survival processing. Journal of Experimental Psychology: Learning, Memory, and 
Cognition, 37, 206-218.

Buss, D. M. (1991). Evolutionary personality psychology. Annual Review of Psychology, $42,459-491$.

Buss, D. M. (1998). Sexual strategies theory: Historical origins and current status. The Journal of Sex Research, 35, 19-31.

Butler, A. C., Kang, S. H. K., \& Roediger III, H. L. (2009). Congruity effects between materials and processing tasks in the survival processing paradigm. Journal of Experimental Psychology: Learning, Memory, and Cognition, 6, 1477-1486.

Caldwell, J. I. \& Masson, M. E. (2001). Conscious and unconscious influences of memory for object location. Memory \& Cognition, 29, 285-295.

Chen, W., Liu, C. H., \& Nakabayashi, K. (2012). Beauty hinders attention switch in change detection: The role of facial attractiveness and distinctiveness. PLOS ONE, 7, e32897; doi: 10.1371/journal.pone.0032897

Chiappe, D., Brown, A., \& Dow, B. (2004). Cheaters are looked at longer and remembered better than cooperators in social exchange situations. Evolutionary Psychology, 2, 108-120.

Chiappe, D., \& MacDonald, K. (2005). The evolution of domain-general mechanisms in intelligence and learning. The Journal of General Psychology, 132, 5-40.

Chun, M. M. \& Turk-Browne, N. B. (2007). Interactions between attention and memory. Current Opinion in Neurobiology, 17, 177-184.

Cook, M., \& Mineka, S. (1989). Observational conditioning of fear to fear-relevant versus fear-irrelevant stimuli in Rhesus monkeys. Journal of Abnormal Psychology, 98, 448-459. 
Cosmides, L. (1989). The logic of social exchange: Has natural selection shaped how humans reason? Studies with the Wason selection task. Cognition, 31, 187-276.

Cosmides, L. \& Tooby, J. (2013). Evolutionary Psychology: New perspectives on cognition and motivation. Annual Review of Psychology, 64, 201-229.

Cosmides, L., Tooby, J., \& Barkow, J. H. (1992). Introduction: Evolutionary Psychology and conceptual integration. In J. H. Barkow, L. Cosmides, \& J. Tooby (Eds.), The Adapted Mind: Evolutionary Psychology and the Generation of Culture (pp. 315). New York, NY: Oxford University Press.

Craik, F. I. \& McDowd, J. M. (1987). Age differences in recall and recognition. Journal of Experimental Psychology: Learning, Memory, and Cognition, 13, 474-479.

Cruciani, F., Berardi, A., Cabib, S., \& Conversi, D. (2011). Positive and negative emotional arousal increases duration of memory traces: Common and independent mechanisms. Frontiers in Behavioral Neuroscience, 5, 1-8.

Dawkins, R. (1976). The Selfish Gene. New York: Oxford University Press.

De Goede, M. \& Postma, A. (2008). Gender differences in memory for objects and their locations: A study on automatic versus controlled encoding and retrieval contexts. Brain and Cognition, 66, 232-242.

DeCaro, M.S., \& Beilock, S. L. (2010). The benefits and perils of attentional control. In B. Bruya and M. Csikszentmihalyi (Eds.), Effortless attention: A new perspective in the cognitive science of attention and action. Cambridge, MA: MIT Press.

DeLoache, J. S. \& LoBue, V. (2009). The narrow fellow in the grass: Human infants associate snakes and fear. Developmental Science, 12, 201-207.

Drewes, J., Trommershäuser, J., Gegenfurtner, K. R. (2011). Parallel visual search and 
rapid animal detection in natural scenes. Journal of Vision, 11, 1-21.

Eastwood, J.D., Smilek, D., \& Merikle, P.M. (2001). Differential attentional guidance by unattended faces expressing positive and negative emotion. Perception $\&$ Psychophysics, 63, 1004-1013.

Ekman, P. (1992a). Are there basic emotions? Psychological Review, 99, 550-553.

Ekman, P. (1992b). An argument for basic emotions. Cognition and Emotion, 6, 169-200.

Falk, J. H. \& Balling, J. D. (2010). Evolutionary influence on human landscape preference. Environment and Behavior, 42, 479-493.

Fenske, M.J. \& Eastwood, J.D. (2003). Modulation of focused attention by faces expressing emotion: Evidence from flanker tasks. Emotion, 3, 327-343.

Flykt, A. (2006). Preparedness for action: Responding to the snake in the grass. The American Journal of Psychology, 119, 29-43.

Fox, E., Griggs, L., \& Mouchlianitis, E. (2007). The detection of fear relevant stimuli: Are guns noticed as quickly as snakes? Emotion, 7, 691-696.

Frankenhuis, W. E. \& de Weerth, C. (2013). Does early-life exposure to stress shape or impair cognition? Current Directions in Psychological Science, 22, 407-412.

Frith, C. (2009). Role of facial expressions in social interactions. Philosophical transactions of the Royal Society B, 364, 3453-3458.

Garcia, J., \& Koelling, R.A. (1966). Relation of cue to consequence in avoidance learning. Psychonomic Science, 4, 123-124.

Gibson, J. M. \& Bahrey, R. (2005). Modality-specificity effects in priming of visual and auditory word-fragment completion. The Journal of General Psychology, 132, 117-137. 
Gould, S. J., \& Lewontin, R. C. (1979). The spandrels of San Marco and the Panglossian paradigm: a critique of the adaptationist programme. Proceedings of the Royal Society of London B, 205, 581-598.

Gould, S. J. \& Vrba, E. S. (1982). Exaptation - A missing term in the science of form. Paleobiology, 8, 4-15.

Graf, P. \& Schacter, D. L. (1985). Implicit and explicit memory for new associations in normal and amnesic subjects. Journal of Experimental Psychology: Learning, Memory, and Cognition, 11, 501-518.

Hackländer, R. \& Lyle, K. (May, 2012). Where was that snake in the grass? Detecting versus remembering threatening stimuli. Poster presented at the meeting of the Association for Psychological Science, Chicago.

Haist, F., Shimamura, A. P., \& Squire, L. R. (1992). On the relationship between recall and recognition memory. Journal of Experimental Psychology: Learning, Memory, and Cognition, 18, 691-702.

Hamann, S. B., Ely, T. D., Grafton, S. T., \& Kilts, C. D. (1999). Amygdala activity related to enhanced memory for pleasant and aversive stimuli. Nature Neuroscience, 2, 289-293.

Hansen, C. H., \& Hansen, R. D. (1988). Finding the face in the crowd: An anger superiority effect. Attitudes and Social Cognition, 54, 917-924.

Hirst, W., Johnson, M. K., Phelps, E. A., \& Volpe, B. T. (1988). More on recognition and recall in amnesics. Journal of Experimental Psychology: Learning, Memory, and Cognition, 14, 758-762.

Hogenson, G. B. (2001). The Baldwin effect: A neglected influence on C. G. Jung’s 
evolutionary thinking. Journal of Analytical Psychology, 46, 591-611.

Holbrook, J. B., Bost, P. R., \& Cave, C. B. (2003). The effects of study-task relevance on perceptual repetition priming. Memory and Cognition, 31, 380-392.

Howe, M. L. (2011). The adaptive nature of memory and its illusions. Current Directions in Psychological Science, 20, 312-315.

Howe, M.L. \& Derbish, M.H. (2010). On the susceptibility of adaptive memory to false memory illusions. Cognition, 115, 252-267.

Howe, M. L. \& Otgaar, H. (2013). Proximate mechanisms and the development of adaptive memory. Current Directions in Psychological Science, 22, 16-22.

Isbell, L.A. (2006). Snakes as agents of evolutionary change in primate brains. Journal of Human Evolution, 51, 1-35.

Jacoby, L. L. (1991). A process dissociation framework: Separating automatic from intentional uses of memory. Journal of Memory and Language, 30, 513-541.

Jennings, J. M. \& Jacoby, L. L. (1993). Automatic versus intentional uses of memory: Aging, attention, and control. Psychology and Aging, 8, 283-293.

Kaplan, R. \& Herbert, E. J. (1987). Cultural and sub-cultural comparisons in preferences for natural settings. Landscape and Urban Planning, 14, 281-293.

Kensinger, E. A. (2007). Negative Emotion Enhances Memory Accuracy: Behavioral and Neuroimaging Evidence. Current Directions in Psychological Science, 16, 213-218.

Kensinger, E. A., Garoff-Eaton, R. J., \& Schacter, D. L. (2006). Memory for specific visual details can be enhanced by negative arousing content. Journal of Memory and Language, 54, 99-112. 
Kimura, M. (1991). Recent development of the neutral theory viewed from the Wrightian tradition of theoretical population genetics. Proceedings of the National Academy of Sciences, 88, 5969-5973.

Ko, P. C., Duda, B., Hussey, E. P., \& Ally, B. A. (2013). Electrophysiological distinctions between recognition memory with and without awareness. Neuropsychologia, 51, 642-655.

Kostic, B., Cleary, A. M., \& McFarlan, C. C. (2012). Extensions of the survival advantage in memory: Examining the role of ancestral context and implied social isolation. Journal of Experimental Psychology: Learning, Memory, and Cognition, 38, 1091-1098.

Koster, E. H. W., Crombez, G., Van Damme, S., Verschuere, B., \& De Houwer, J. (2004). Does imminent threat capture and hold attention? Emotion, 4, 312-317.

Kroneisen, M. \& Erdfelder, E. (2011). On the plasticity of the survival processing effect. Journal of Experimental Psychology: Learning, Memory, and Cognition, 37, $1553-1562$.

Kuhbander, C., Spitzer, B., \& Pekrun, R. (2011). Read-out of emotional information from iconic memory: The longevity of threatening stimuli. Psychological Science, 22, 695-700.

LeDoux, J. (1998). The emotional brain. New York: Phoenix.

LeDoux, J. (2012). Rethinking the emotional brain. Neuron, 73, 653-676.

Lench, H. C., Flores, S. A., \& Bench, S. W. (2011). Discrete emotions predict changes in cognition, judgment, experience, behavior, and physiology: A meta-analysis of experimental emotion elicitations. Psychological Bulletin, 137, 834-855. 
Lench, H. C., Bench, S. W., \& Flores, S. A. (2013). Searching for evidence, not a war: Reply to Lindquist, Siegel, Quigley, and Barrett (2013). Psychological Bulletin, 139, 264-268.

Li, W., Paller, K. A., \& Zinbarg, R. E. (2008). Conscious intrusion of threat information via unconscious priming in anxiety. Cognition and Emotion, 22, 44-62.

Lindquist, K. A., Siegel, E. H., Quigley, K. S., \& Barrett, L. F. (2013). The hundred-year emotion war: Are emotions natural kinds or psychological constructions? Comment on Lench, Flores, and Bench (2011). Psychological Bulletin, 139, 255263.

Lipp, O. V., Derakshan, N., Waters, A. M., Logies, S. (2004). Snakes and cats in the flower bed: Fast detection is not specific to pictures of fear-relevant animals. Emotion, 4, 233-250.

LoBue, V. (2009). More than just another face in the crowd: Superior detection of threatening facial expressions in children and adults. Developmental Science, 12, 305-313.

LoBue, V. (2010a). What's so scary about needles and knives? Examining the role of experience in threat detection. Cognition \& Emotion, 24, 180-187.

LoBue, V. (2010b). And along came a spider: An attentional bias for the detection of spiders in young children and adults. Journal of Experimental Child Psychology ,107, 59-66.

LoBue, V. (2013). What are we so afraid of? How early attention shapes our most common fears. Child Development Perspectives, 7, 38-42.

LoBue, V. \& DeLoache, J. S. (2008). Detecting the snake in the grass: Attention to fear- 
relevant stimuli by adults and young children. Psychological Science, 19, 284289.

LoBue, V. \& DeLoache, J. S. (2010). Superior detection of threat-relevant stimuli in infancy. Developmental Science, 13, 221-228.

LoBue, V., \& DeLoache, J. S. (2011). What's so special about slithering serpents? Children and adults rapidly detect snakes based on their simple features. Visual Cognition, 19, 129-143.

LoBue, V., Rakison, D. H., \& DeLoache, J. S. (2010). Threat perception across the lifespan: Evidence for multiple converging pathways. Current Directions in Psychological Science, 19, 375-379.

Lyle, K. B., McCabe, D. P., \& Roediger III, H. L. (2008). Handedness is related to memory via hemispheric interaction: Evidence from paired associate recall and source memory tasks. Neuropsychology, 22, 523-530.

Masataka, N., Hayakawa, S., \& Kawai, N. (2010). Human young children as well as adults demonstrate, superior' rapid snake detection when typical striking posture is displayed by the snake. PLOS ONE, 5; doi: 10.1371/journal.pone.0015122.

Mather, M. (2007). Emotional arousal and memory binding: An object-based framework. Perspectives on Psychological Science, 2, 33-52.

Mather, M. \& Nesmith, K. (2008). Arousal-enhanced location memory for pictures. Journal of Memory and Language, 58, 449-464.

Mather, M. \& Sutherland, M. R. (2011). Arousal-biased competition in perception and memory. Perspectives on Psychological Science, 6, 114-133.

Mayr, E. (2001). What Evolution Is. New York: Basic Books. 
McDaniel, M. A., Lyle, K. B, Butler, K., M., \& Dornburg, C. C. (2008). Age-related deficits in reality monitoring of action memories. Psychology and Aging, 23, 646656.

Mealey, L., Daood, C., \& Krage, M. (1996). Enhanced memory for faces of cheaters. Ethology \& Sociobiology, 17, 119-128.

Mehl, B. \& Buchner, A. (2008). No enhanced memory for faces of cheaters. Evolution \& Human Behavior, 29, 35-41.

Nairne, J. S. (2010). Adaptive memory: Evolutionary constraints on remembering. Psychology of Learning and Motivation, 53, 1-32.

Nairne, J. S., Thompson, S. R., \& Pandeirada, J. N. S. (2007). Adaptive memory: Survival processing enhances retention. Journal of Experimental Psychology: Learning, Memory, \& Cognition, 33, 263-273.

Nairne, J. S. \& Pandeirada, J. N. S. (2008). Adaptive memory: Remembering with a Stone-Age brain. Current Directions in Psychological Science, 17, 239-243.

Nairne, J. S., Pandeirada, J. N. S., \& Thompson, S. R. (2008). Adaptive memory: The comparative value of survival processing. Psychological Science, 19, 176-180.

Nairne, J. S., Van Arsdall, J. E., Pandeirada, J. N. S., \& Blunt, J. R. (2012). Adaptive memory: Enhanced location memory after survival processing. Journal of Experimental Psychology: Learning, Memory, and Cognition, 38, 495-501.

Naveh-Benjamin, M., Shing, Y. L., Kilb, A., Werkle-Bergner, M., Lindenberger, U., \& Li, S. (2009). Adult age differences in memory for name-face associations: The effects of intentional and incidental learning. Memory, 17, 220-232.

New, J., Cosmides, L., \& Tooby, J. (2007). Category-specific attention for animals 
reflects ancestral priorities, not expertise. Proceedings of the National Academy of Sciences, 104, 16598-16603.

Öhman, A., Eriksson, A., \& Olofsson, C. (1975). One trial learning and superior resistance to extinction of autonomic responses conditioned to potentially phobic stimuli. Journal of Comparative and Physiological Psychology, 88, 619-627.

Öhman, A., Flykt, A., \& Esteves, F. (2001). Emotion drive attention: Detecting the snake in the grass. Journal of Experimental Psychology: General, 130, 466-478.

Öhman, A., Fredrikson, M., Hugdahl, K., \& Rimmö, P. A. (1976). The premise of equipotentiality in human classical conditioning: Conditioned electrodermal responses to potentially phobic stimuli. Journal of Experimental Psychology: General, 103, 313-337.

Öhman, A., Lundqvist, D., \& Esteves, F. (2001). The face in the crowd revisited: A threat advantage with schematic stimuli. Journal of Personality and Social Psychology, 80, 381-396.

Öhman, A. \& Mineka, S. (2001). Fears, phobias, and preparedness: Toward an evolved module of fear and fear learning. Psychological Review, 108, 483-522.

Oldfield, R. (1971). The assessment and analysis of handedness: The Edinburgh Inventory. Neuropsychologia, 9, 97-113.

Patel, R., Girard, T. A., Green, R. E. A. (2012). The influence of indirect and direct emotional processing on memory for facial expressions. Cognition and Emotion, $26,1143-1152$.

Penkunas, M. J. \& Coss, R. G. (2013). Rapid detection of visually provocative animals by preschool children and adults. Journal of Experimental Child Psychology, 114, 
522-536.

Purcell, D. G., Stewart, A. L., \& Skov, R. B. (1996). It takes a confounded face to pop out of a crowd. Perception, 25, 1091-1108.

Rakison, D. H., \& Derringer, J. (2008). Do infants possess an evolved spider-detection mechanism? Cognition, 107, 381-393.

Roediger III, H. L. (1990). Implicit memory: Retention without remembering. American Psychologist, 45, 1043-1056.

Rugg, M. D., Mark, R. E., Walla, P., Schloerscheidt, A. M., Birch, C. S., \& Allan, K. (1998). Dissociation of the neural correlates of implicit and explicit memory. Nature, 392, 595-598.

Sadeh, T., Maril, A., \& Goshen-Gottstein, Y. (2012). Encoding-related brain activity dissociates between the recollective processes underlying successful recall and recognition: A subsequent-memory study. Neropsychologia, 50, 2317-2324.

Schacter, D. L. (1987). Implicit memory: History and current status. Journal of Experimental Psychology: Learning, Memory, and Cognition, 3, 501-518.

Schacter, D. L. (1992). Priming and multiple memory systems: Perceptual mechanisms of implicit memory. Journal of Cognitive Neuroscience, 4, 244-256.

Schacter, D. L. (1996). Searching for memory: The brain, the mind, and the past. New York: Basic Books.

Schacter, D. L., Harbluk, J. A., \& McLachlan, D. R. (1984). Retrieval without recollection: An experimental analysis of source amnesia. Journal of Verbal Learning and Verbal Behavior, 23, 593-611.

Schneider, W., Eschmann, A., \& Zuccolotto, A. (2002). E-Prime user's guide. Pittsburgh, 
PA: Psychology Software Tools, Inc.

Schott, B. H., Henson, R. N., Richardson-Klavehn, A., Becker, C., Thoma, V., Heinze, H-J., \& Düzel, E. (2005). Redefining implicit and explicit memory: The functional neuroanatomy of priming, remembering, and control of retrieval. Proceedings of the National Academy of Sciences of the United States of America, 102, 1257-1262.

Schmidt, S. R. (2012). Memory for emotional words in sentences: The importance of emotional contrast. Cognition and Emotion, 26, 1015-1035.

Seligman, M. E. (1971). Phobias and preparedness. Behavior Therapy, 2, 307-320.

Shariff, A. F. \& Tracy, J. L. (2011a). What are emotion expressions for? Current Directions in Psychological Science, 20, 395-399.

Shariff, A. F. \& Tracy, J. L. (2011b). Emotion expressions: On signals, symbols, and spandrels-A response to Barrett (2011). Current Directions in Psychological Science, 20, 407-408.

Skrupskelis, I. K. (2007). Evolution and pragmatism: An unpublished letter of William James. Transactions of the Charles S. Peirce Society, 43, 745-752.

Symons, C. S. \& Johnson, B. T. (1997). The self-reference effect in memory: A metaanalysis. Psychological Bulletin, 121, 371-394.

Tulving, E. \& Watkins, M. J. (1973). Continuity between recall and recognition. American Journal of Psychology, 86, 739-748.

van den Berg, A. E., Hartig, T., \& Staats, H. (2007). Preference for nature in urbanized societies: Stress, restoration, and the pursuit of sustainability. Journal of Social Issues, 63, 79-96. 
Voss, J. L. \& Paller, K. A. (2008). Brain substrates of implicit and explicit memory: The importance of concurrently acquired neural signals of both memory types. Neurospcyhologia, 46, 3021-3029.

Wason, P. (1966). In B. M. Foss (Ed.), New horizons in psychology (pp. 135-151). Harmondsworth: Penguin.

Weinstein, Y., Bugg, J. M., \& Roedeiger III, H. L. (2008). Can the survival recall advantage be explained by basic memory processes? Memory \& Cognition, 36, 913-919.

Weldon, M. S. \& Roediger III, H. L. (1987). Altering retrieval demands reverses the picture superiority effect. Memory \& Cognition, 15, 269-280.

West-Eberhard, M. J. (1989). Phenotypic plasticity and the origins of diversity. Annual Review of Ecology and Systematics, 20, 249-278.

Wilson, S., Darling, S., \& Sykes, J. (2011). Adaptive memory: fitness relevant stimuli show a memory advantage in a game of pelmanism. Psychonomic Bulletin and Review, 18, 781-786.

Wilson-Mendenhall, C. D., Barrett, L. F., \& Barsalou, L. W. (2013). Neural evidence that human emotions share core affective properties. Psychological Science, 24, 947956.

Winograd, E. (1981). Elaboration and distinctiveness in memory for faces. Journal of Experimental Psychology: Human Learning and Memory, 7, 181-190.

Yang, J., Cao, Z., Xu, X., \& Chen, G. (2012). The amygdala is involved in affective priming effect for fearful faces. Brain and Cognition, 80, 15-22.

Yang, J., Xu, X., Du, X., Shi, C., \& Fang, F. (2011). Effects of unconscious processing 
on implicit memory for fearful faces. PLoS ONE, 6, e14641. Doi:

10.1371/journal.pone.0014641

Yonelinas, A. P. (1999). The contribution of recollection and familiarity to recognition and source-memory judgments: A formal dual-process model and an analysis of receiver operating characteristics. Journal of Experimental Psychology: Learning, Memory, and Cognition, 6, 1415-1434. 
APPENDIX A

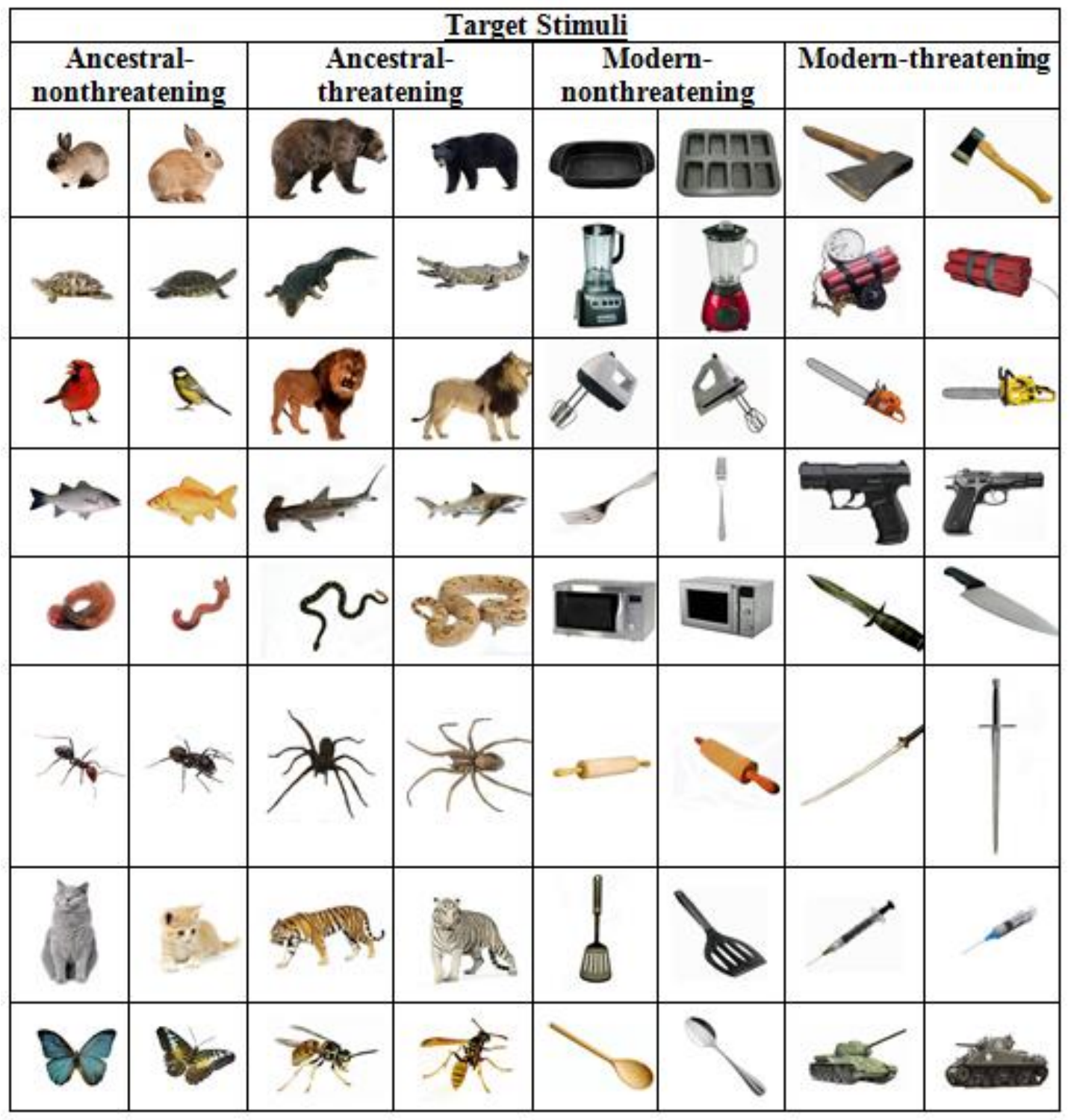




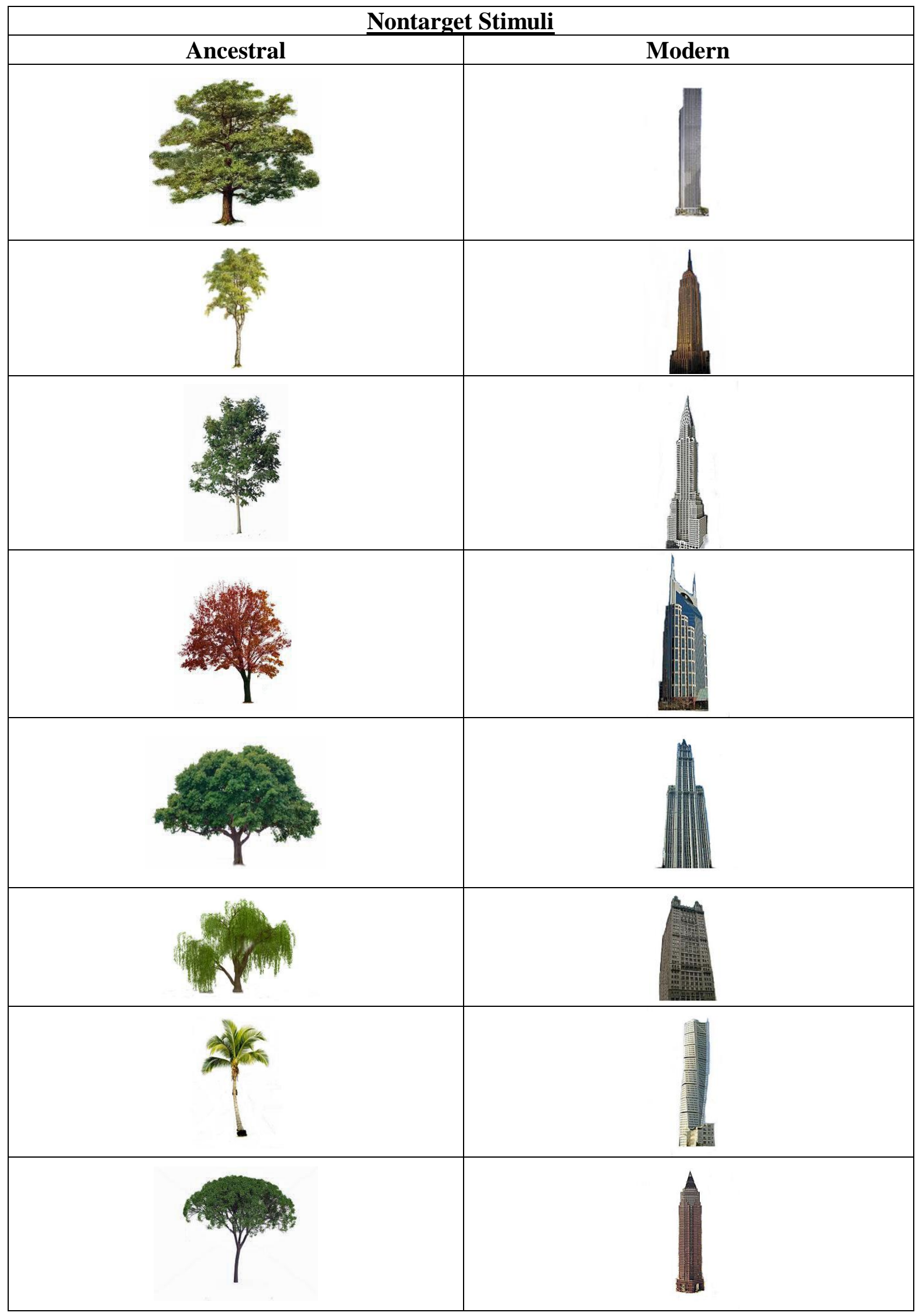




\section{APPENDIX B}

1) Incidental Condition - 1/3 subjects are only told how to perform attention task, they get no special instructions about memory task

a. You will now see a series of screens each containing four pictures. Your task is to determine whether all of the pictures come from the same category or if one of the pictures comes from a different category. The pictures will either be from a tree category, building category, or some other category. If all pictures came from the same category press the " $\mathrm{f}$ " key. If one picture comes from a different category press the " $\mathrm{j}$ " key. Try and complete the task as quickly as you can while still being accurate.

2) Intentional Condition - $1 / 3$ subjects get "Intentional" instructions

a. You will now see a series of screens each containing four pictures. Your task is to determine whether all of the pictures come from the same category or if one of the pictures comes from a different category. The pictures will either be from a tree category, building category, or some other category. If all pictures came from the same category press the " $\mathrm{f}$ " key. If one picture comes from a different category press the " $\mathrm{j}$ " key If you see a picture that comes from a category other than trees or buildings do your best to remember where you see it because your memory for its location will be tested later. Try and complete the task as quickly as you can while still being accurate.

3) Survival Condition - 1/3 subjects get "Survival" instructions

a. You will now see a series of screens each containing four pictures. Your task is to determine whether all of the pictures come from the same category or if one of the pictures comes from a different category. The pictures will either be from a tree category, building category, or some other category. Some of the pictures will be more relevant to your survival than others. If all pictures came from the same category press the " $\mathrm{f}$ " key. If one picture comes from a different category press the " $\mathrm{j}$ " key. If you see a picture that comes from a category other than trees or buildings do your best to remember where you see it because your memory for its location will be tested later. Remembering the locations of certain objects can help 
b. you survive in the wild because you will be able to either find or avoid them in the future. Try and complete the task as quickly as you can while still being accurate. 


\title{
CURRICULUM VITAE
}

\author{
Ryan P. Hackländer \\ University of Louisville \\ Phone: +4915119609594 \\ Department of Psychological and Brain \\ Sciences \\ Email: rpmulv01@louisville.edu \\ ryanhacklaender@yahoo.com \\ Louisville, KY 40292
}

\section{Education}

Current

spring 2013

Ph.D. program in experimental psychology; expected graduation

University of Louisville

Advisor: Keith B. Lyle

2010

M.S. in Experiment Psychology

University of Louisville

Advisor: Keith B. Lyle

2008

B.S. in Psychology; Kentucky Wesleyan College

\section{Professional Experience}

Spencerian College

2012 - 2012 Substitute Professor, Department of Business, Dr. Tonnie Renfro

\section{University of Louisville}

2010 - $2012 \quad$ Teaching Assistant, Department of Psychological and Brain

Sciences

2010 - 2012 Research Assistant, Memory and Cognition Lab, Dr. Keith B. Lyle

Western Kentucky University

Summer $2006 \quad$ Undergraduate Research Assistant, REST Lab, Dr. Pitt Derryberry 


\section{Honors and Awards}

$2008-2010 \quad$ Graduate Student Fellowship, University of Louisville

2008

Who's Who Among American University Students

2008

Rex O’Flynn Psychology Award, Kentucky Wesleyan College

2008

Alpha Chi, Kentucky Wesleyan College

2007

Psi Chi, Kentucky Wesleyan College

2006

NSF funded REU award, Western Kentucky University

\section{Publications}

Lyle, K.B., Hanaver-Torrez, S.D., Hackländer, R.P., \& Edlin, J.M. (2011).

Consistency of handedness, regardless of direction, predicts baseline memory accuracy and potential for memory enhancement. Journal of Experimental Psychology: Learning, Memory, and Cognition. doi: 10.1037/a0024831

Derryberry, W.P., *Mulvaney, R., Brooks, J., \& Chandler, C. (2009). Addressing the Relationships Among Moral Judgment Development, Authenticity, Nonprejudice, and Volunteerism. Ethics and Behavior, 19, 201-217.

\section{Conference Presentations}

Lyle, K.B., \& Hackländer, R.P. (2013). Seeing the truth: Saccade execution increases correct "true" responses on T/F history tests. Poster presented at the fifty-fourth annual meeting of the Psychonomic Society. Toronto, Canada.

Hackländer, R.P. \& Lyle, K.B. (2012). Where was that snake in the grass? The locations of threatening stimuli are detected faster but not remembered better. Poster presented at the annual meeting of the Association for Psychological Science. Chicago, Illinois.

Hackländer, R.P. \& Lyle, K.B. (2010). Do all bilateral motor movements enhance memory retrieval? Poster presented at the annual meeting of the Midwestern Psychological Association. Chicago, Illinois.

Derryberry, W.P., Barger, B., Brooks, J., \& *Mulvaney, R. (2007). The effect of affect: Exploring the relevance of sadness and anger to macromoral reasoning 
and judgment. Paper presented at the annual meeting of the American Educational Research Association. Chicago, Illinois.

Derryberry, W.P., *Mulvaney R., Brooks, J., \& Chandler, C. (2007). Addressing the relationships among authenticity, nonprejudice, moral judgment and volunteerism. Paper presented at the annual meeting of the American Educational Research Association. Chicago, Illinois. 